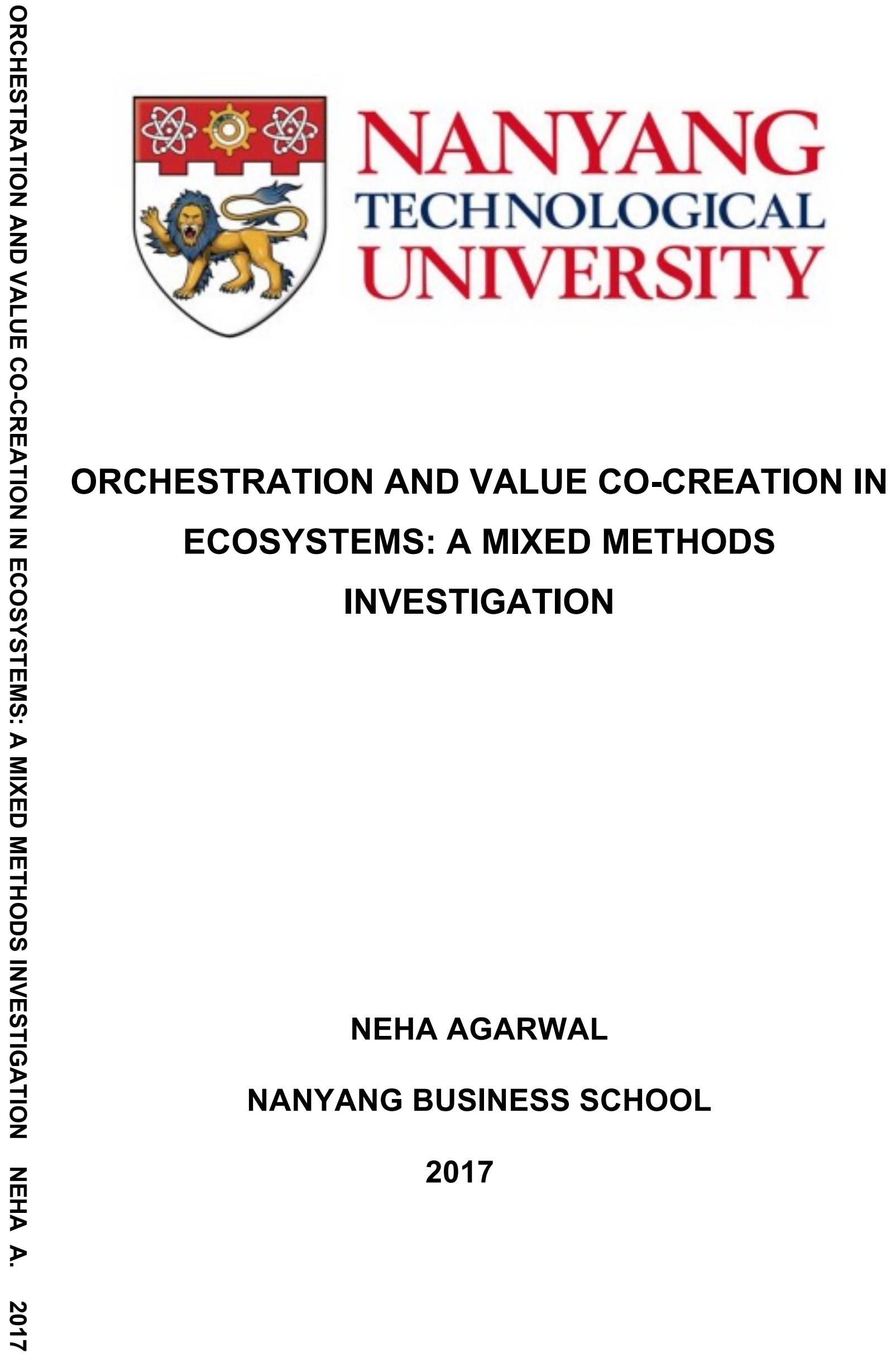





\section{ORCHESTRATION AND VALUE CO-CREATION IN ECOSYSTEMS: A MIXED METHODS INVESTIGATION}

Nanyang Business School

A thesis submitted to the Nanyang Technological University in partial fulfillment of the requirement for the degree of Doctor of Philosophy 



\section{ACKNOWLEDGEMENTS}

As I arrive on this important juncture, I would like to express my sincere gratitude to many extremely important people without whose support, the journey so far would have been highly inconceivable.

I am highly indebted and thankful to my supervisor, Professor Christina Soh for her patience and support in mentoring my research. When I first started, I was confounded and overwhelmed with the nature of conducting business research. Professor Soh's handholding in my initial years and empowering me thereafter has been extremely instrumental in building my foundation on how a research should be conducted. She has truly inspired me for doing hard work, developing conceptual clarity and working in collaborations with others.

I am also grateful to my co-supervisor, Dr. Adrian Yeow who has offered valuable insights and suggestions to my research. His academic expertise, data resources and constructive feedback have helped me evolve my research towards a better and defining contribution. I am very indebted that he has mentored me in this journey and hope to learn more from him going forward.

I would also like to express my sincere thanks to Dr. Goh Kim Huat for his very patient responses to my numerous queries and requests. As I start to imbibe new methodologies in research, I am very fortunate to be guided by his academic experience. I hope to benefit and continue to learn more from his expertise and guided support.

I would like to thank all the faculty at business school who have taken out their valuable time to teach, provide feedback and share their experiences with me all along. They have broadened by arena of research, introduced me to different methodologies and made this learning experience an enriching one. Special thanks to Dr. Sia Siew Kein for his tremendous support, valuable insights and also for helping me out in the formative years of my research. 
Also, I take this as an opportunity to thank my colleagues and friends Anjali, Avijit, Geeta and Lin Yan who have tremendously helped me cope up with the highs and lows of a doctoral life. Their support has no doubt strengthened my commitment and made me a stronger person than I was. I would also like to thank NBS PhD office for being so calm and helpful in all the administrative processes throughout these years.

Finally, this thesis has not been possible without the innumerous sacrifices made by my parents, my brother and my husband and their unconditional support all the way. Their comforting hand has always melted my worries and motivated me to move on. Thanks for always being there for me. This was just impossible without you. 


\section{TABLE OF CONTENTS}

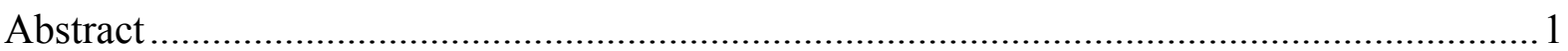

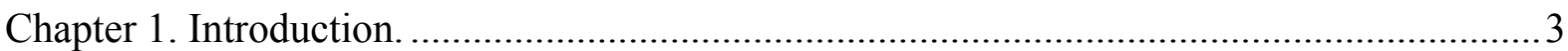

Chapter 2. Tensions in Ecosystem Evolution: Evidence from Integrated Care........................ 14

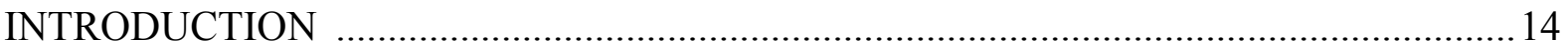

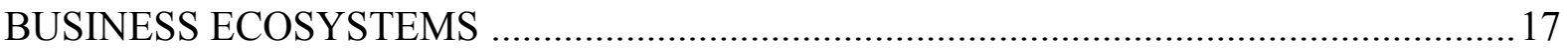

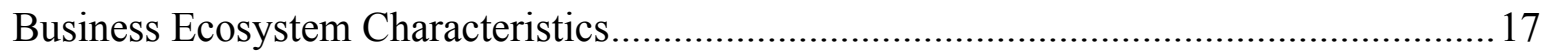

Business Ecosystem Evolution and Role of the Keystone Organization ............................ 18

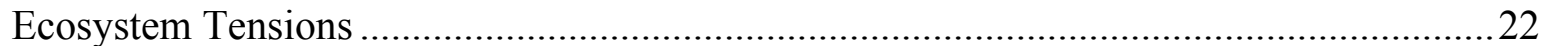

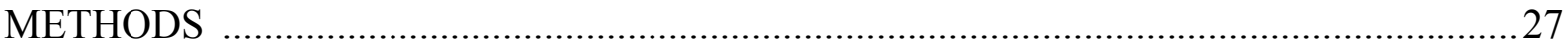

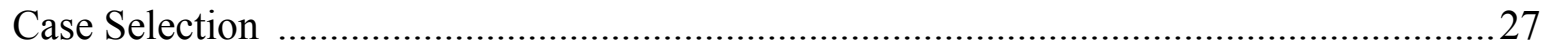

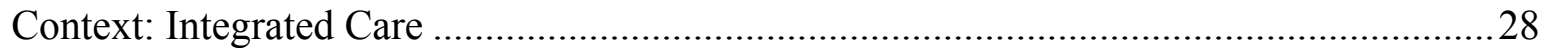

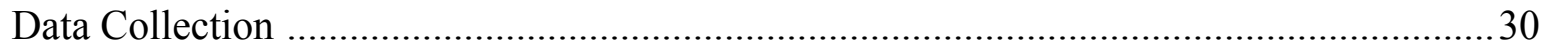

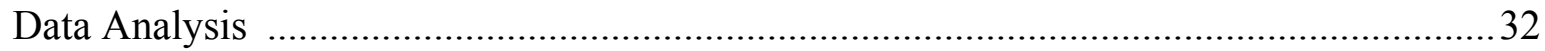

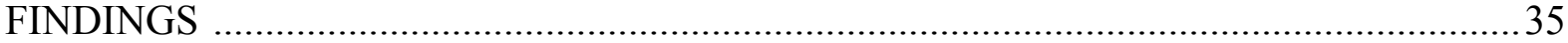

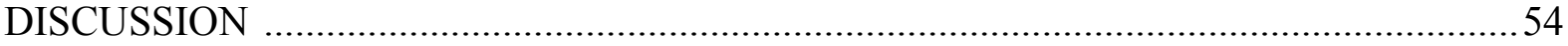

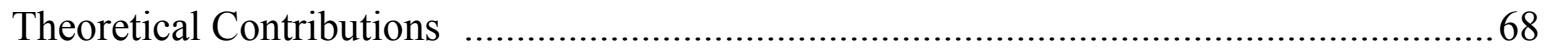

Practical Implications

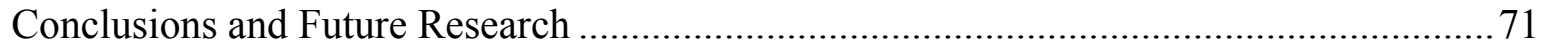

Chapter 3. Value Co-creation in Service Ecosystems: A Member's Perspective.....................73

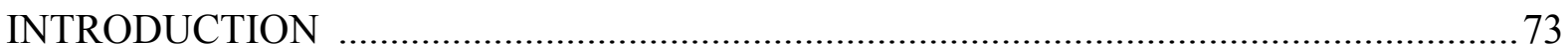

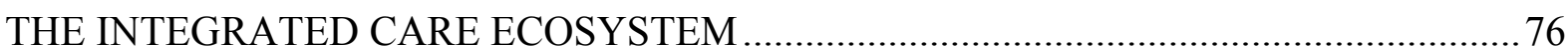


Service Exchanges and Value to Members

Resource Exchanges and Value to Members .83

Impact of Relational Exchanges on Service Exchanges and Resource Exchanges 85

METHODS AND MEASURES

Research Context 88

Data Collection

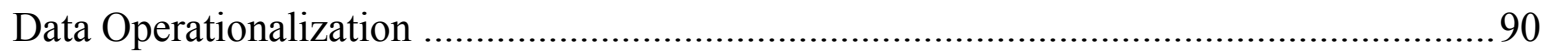

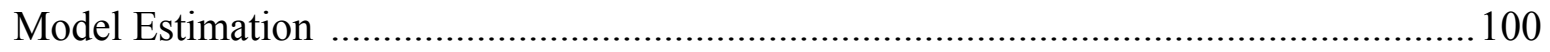

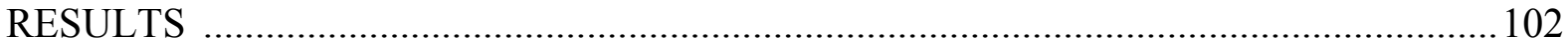

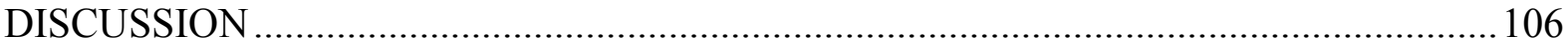

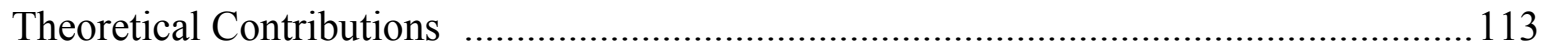

Practical Implications

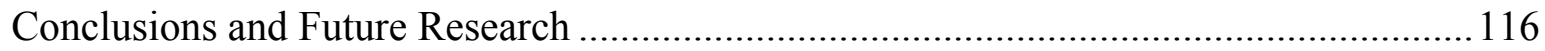

Chapter 4. Summary And Implications of Findings ...................................................... 118

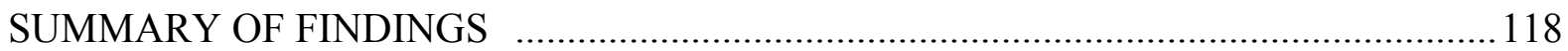

CONTRIBUTIONS TO THEORY

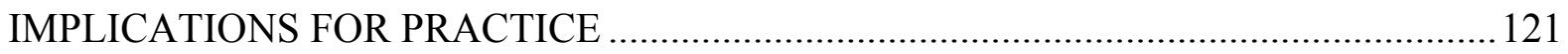

Implications for the Keystone Organization and the Members ...................................... 121

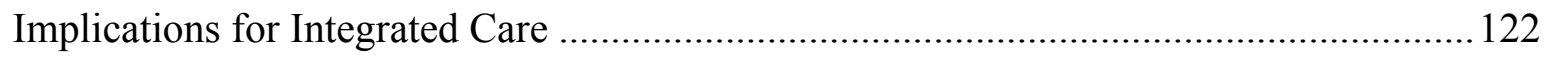

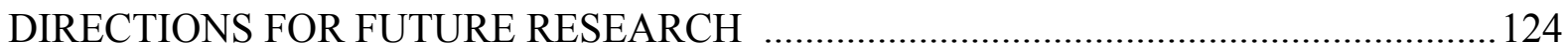

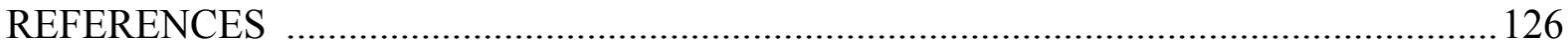




\section{List of Figures}

Chapter 2

Figure 1. Ecosystem Evolution

Chapter 3

Figure 1. Research Model

Figure 2. Research Model Results 106

\section{List of Tables}

Chapter 2

Table 1. Ecosystem Evolution and Keystone Organization's Orchestration Actions 21

Table 2. Interviews 32

Table 3. Member Enrollment: Tensions and Adaptive Orchestration 64

Table 4. Joint Capabilities Development: Tensions and Adaptive Orchestration 65

Table 5. Ecosystem Renewal: Tensions and Adaptive Orchestration 67

Chapter 3

Table 1. Summary of Descriptive Statistics 98

Table 2. Correlations 99

Table 3(a). Results- H1 \& H2- Clinical Outcomes 103

Table 3(b). Results- H1 \& H2- User Satisfaction 104

Table 3(c). Results - H1 \& H2- Cost Effectiveness 104

Table 4. Results- H3 \& H4 105

Table 5. Summary of Hypothesis 105 


\begin{abstract}
Organizations today are increasingly joining ecosystems to avert the threat of extinction as well as to generate more value by harnessing capabilities of other organizations. However, sustainability of such ecosystems has been an ongoing concern. On the one hand, there are challenges during ecosystem evolution which need to be addressed by the keystone organization and on the other hand, members have limited perspective on how their engagement in value co-creational exchanges with the keystone organization can result in continued value for them from the ecosystems. This dissertation takes a mixed methods approach to conduct two studies which explore these two critical aspects of ecosystemstensions in ecosystem evolution and value to members.

The first study conducts an in-depth case analysis to explore how the keystone organization manages the evolution of an ecosystem by addressing the emerging tensions. Our findings highlight three tensions that exist during three phases of ecosystem evolution member enrollment, joint capabilities development, and ecosystem renewal. Our findings also reveal that in addition to the routine orchestration actions which are initiated and driven by the keystone organization to orchestrate evolution, the keystone organization also engages in actions that are in response to the tensions and are taken in consideration with the members' feedback. These adaptive orchestration actions complement the routine actions for sustainable evolution of the ecosystem.
\end{abstract}

The second study conducts a panel data analysis with fixed-effects to examine how the value co-creation exchanges among the members and the keystone organization influence the value to members over time. Taking a service-dominant view to ecosystems, we explicate three value co-creational exchanges, namely, service exchanges, resource exchanges and relational exchanges that occur between members and the keystone organization and then examine the influence on value to members (economic and non economic). Our findings 
suggest that participation in different value co-creational exchanges, can bring a wider range of benefits to members, measured beyond changes in their market values.

Together, these studies highlight important considerations for building a sustainable ecosystem. While study 1 effectively brings out the role of keystone organization in orchestrating a sustainable ecosystem, study 2 surfaces how members can derive a wide range of value by engaging in value co-creational exchanges with the keystone organization. 


\section{CHAPTER 1.}

\section{INTRODUCTION}

Ecosystems are becoming increasingly prevalent as organizations are seeking collaborations to avoid threat of extinction as well as to generate value which they cannot create on their own (Adner 2006). A business ecosystem is defined as a "community of interacting entities - organizations and individuals (including customers) that coevolve their capabilities and roles and depend on one another for their overall effectiveness and survival" (Iansiti and Levien 2004; Lusch and Nambisan 2015, pp. 161; Moore 1993). Organizations such as Microsoft, Google, Alibaba and Amazon today are leading vast ecosystems by creating technology platforms which in turn are being leveraged by thousands of organizations across the world. By harnessing each other's capabilities, these organizations are co-creating value by accessing new markets and creating holistic solutions for the customers (Adner 2006; Iansiti and Levien 2004). For example, Alibaba, which started as a small e-commerce startup in China, has developed a robust ecosystem with millions of small and medium size organizations delivering services to more than 80 million users (Tan et al. 2015). Similarly, Amazon which started as a cyber book-selling platform in 1994 developed its own ecosystem through Amazon Associates Program allowing its affiliates to sell products on its existing technology platform. Not only did Amazon expand its product line, it continued to evolve by offering additional marketing and web services for its partners.

Ecosystems are characterized by diverse members which are highly interdependent, share symbiotic relationships and are held together by a shared purpose. While members are diverse in their visions, cultures and business processes, these share high interdependencies as performance of one member is often dependent on the capabilities of other members (Adner and Kapoor 2010; Iansiti and Levien 2004). To manage such diverse set of members, the ecosystem is orchestrated by a keystone organization which develops a shared purpose, 
joint capabilities and governance mechanisms to facilitate co-creation of value in form of products and services (Kandiah and Gossain 1998). The keystone organization often also develops a common platform to foster exchanges among different members. Members leverage these platforms to interact with other peer members for problem solving, knowledge sharing and learning (Jha et al. 2016; Tan et al. 2015). They can also seek collaboration with each other to develop more specialized skills or provide holistic solutions to their customers (Wareham et al. 2014). Better interactions among the members also benefits the keystone organization. For example, Alibaba fostered greater interactions among its merchants and buyers on its technology platform which in turn helped it to analyze and predict their future needs (Srivastava and Shainesh 2015; Tan et al. 2015).

Ecosystems operate in a highly complex environmental context as their members often span across different industries (Moore 1993; Reeves et al. 2016). As these members share high interdependencies, challenges such as changes in government regulations, consumer preferences or innovation in technology (Moore 1993; Reeves et al. 2016) in one part of the ecosystem can quickly cascade to the entire ecosystem giving rise to both opportunities and threats (Weill and Woerner 2015). While opportunities manifest in form of expansion to new markets and cross-selling of products and services, threats may result in loss of members or rise of competitive ecosystems.

Ecosystems also faces internal challenges due to their very inherent characteristic of member diversity and the need to align their goals and efforts to the shared ecosystem purpose (Banoun et al. 2016; Garud et al. 2002; Lindgren et al. 2015; Wareham et al. 2014). For example, profit seeking behavior from members may jeopardize the co-creation of value in ecosystems (Banoun et al. 2016; Garud et al. 2002; Lindgren et al. 2015; Wareham et al. 2014). 
Challenges from technological change, competition or internal diversity often reveal tensions in organizations (Garud et al. 2002; Lewis 2000). The broader organizational literature takes a paradox lens to define tensions as contradictory demands such as individual vs collective or collaboration vs control which need to be addressed simultaneously to meet long term sustainability (Banoun et al. 2016; Smith and Lewis 2011). However, these studies have exemplified tensions at organizational level. Following this, and the recent studies in ecosystems that have noted tensions (Garud et al. 2002; Lindgren et al. 2015; Wareham et al. 2014), we explore this concept in ecosystems where challenges from environmental context and internal diversity often manifest as tensions.

For example, an often noted tension in ecosystems is individual vs collective identifications which stems from the differences in expectations and identities of the members. The diverse members each have their own identity, yet need to simultaneously embrace the collective ecosystem identity. For instance, Banoun et al (2016) in their study of service ecosystems focus on the individual vs collective tension arising from different expectations of members over time. The findings show that in the first phase, tension becomes salient as some members focus on the technical aspects while others insist on traditional procedures. To balance this tension, the keystone organization had to clarify the relationships though communication and standardization of processes. As the ecosystem evolved to the next phase, tension arose again as now the members felt the standard industrial procedures were too rigid and lacked market orientation which adversely affected their clients. Hence again the keystone organization introduced temporary changes to meet client expectations. As the keystone organization responded to the tension, it led to further evolution of the ecosystem over time (Banoun et al. 2016; Garud et al. 2002; Lindgren et al. 2015). 
However, this evolution is challenging and if not addressed appropriately, may even result in the collapse of the ecosystem as all components of an ecosystem may not evolve at the same rate or some may choose not to evolve at all (Adner 2006; Reeves et al. 2016). For example, if the keystone organization is unable to provide continued value for its members or is unable to deal with environmental changes, the members may defect to other competing ecosystems. Perhaps, the most striking examples include those of Eastman Kodak and Symbian ecosystems which declined after failure to deal with some of these challenges. For instance in the case of Symbian ecosystem, some members like Samsung and ARM chose to evolve with the changing context, however Symbian as the keystone organization itself chose not to evolve (West and Wood 2013). This eventually led to the demise of the Symbian ecosystem. Hence, sustainability of ecosystems over time has become a matter of concern especially for the keystone organization which has to orchestrate the ecosystem development so that all the components co-evolve together through mutual and reciprocal actions.

Furthermore, this sustainability is also threatened when members are unable to derive value from participation in ecosystems. Since organizations in an ecosystem share high dependencies, ecosystems require reciprocal and mutual exchanges among the members and the keystone organization to co-create value. This suggests that merely joining an ecosystem would not necessarily culminate into benefits for the members. Rather, the ability of members to derive value from the ecosystem is shaped by their value co-creational exchanges with the keystone organization. It hence becomes a vital issue from member's perspective to understand how they can derive value from participation in an ecosystem.

Research in IS literature has started to explore these two critical aspects of business ecosystems. First set of studies explore the evolution, tensions or challenges and the role of the keystone organization in ecosystems (Jha et al. 2016; Leong et al. 2016; Srivastava and Shainesh 2015; Tan et al. 2015) and the second stream explores the co-creation of value and 
business performance of organizations participating in ecosystems (Adner and Kapoor 2010; Ceccagnoli et al. 2012; Han et al. 2012; Rai et al. 2012; Sarker et al. 2012). However, considerable gaps continue to exist on how ecosystems evolve by addressing accompanying tensions as well as on how member's participation in ecosystems affects the value they accrue over time.

This dissertation takes a mixed methods approach to address some of these gaps in the ecosystem literature. First, there is limited understanding on the tensions faced by ecosystems as they evolve over time. Prior research dealing with ecosystem evolution (Jha et al. 2016; Leong et al. 2016; Srivastava and Shainesh 2015; Tan et al. 2015) has identified three critical phases of ecosystem evolution, which we term, member enrollment, joint capabilities development and ecosystem renewal, based on our understanding of the key focus in each phase. A recent stream has also suggested that tensions exist in ecosystems often due to diversity of members (Garud et al. 2002; Lindgren et al. 2015; Wareham et al. 2014) such as individual vs collective identifications and autonomy vs control. These studies have either observed a single tension or tensions at only specific points of time. We however suspect that tensions unfold with evolution and may not be equally salient at all points in time. For instance, while diversity of members may be a critical tension in the earlier phases of ecosystem, latter phases may be challenged by external environmental changes. Many studies currently do not address this emergent view of tensions over time.

Second, since tensions have not been observed over time, how the keystone organization manages these tensions is also not well understood. Past research has highlighted that the keystone organization plays a critical role in the evolution of the ecosystem (Jha et al. 2016; Leong et al. 2016; Srivastava and Shainesh 2015; Tan et al. 2015; Zeng 2015). In each phase of the evolution, the keystone organization engages in a series of orchestration actions (purposeful and deliberate actions) such as monitoring ecosystem 
performance or expanding the ecosystem through new members or services that bring it closer to the shared purpose While these standard routine actions are necessary to evolve the ecosystem in the right direction, they may not be sufficient alone to deal with the accompanying tensions. For example, Tan et al (2015) in their study on Alibaba's ecosystem touch upon some aspect of such orchestration actions in one of the phases, where the keystone organization (Alibaba in this case) countered the threat from other ecosystems by increasing interactions among its members. This action was specifically taken to address the challenge faced by the ecosystem. There is hence need for focused research on how the keystone organization manages the emerging tensions during evolution. If these tensions remain unattended, these may result in unsustainability of the ecosystem (Reeves et al. 2016). In the first study, therefore we address the following research question

\section{RQ1: How does the keystone organization manage the evolution of an ecosystem by}

\section{addressing the emerging tensions?}

To study this research question, we adopted a case study methodology as it allowed us to surface emerging tensions during ecosystem evolution as well as the related keystone organization actions.

We examine this question in the context of integrated care where diverse medical providers form an ecosystem to deliver seamless healthcare to people with multiple care needs. The changes in healthcare context such as rising costs, rapidly aging population and manpower shortage have resulted in both opportunities (technology and innovation) and threats (high demands) providing impetus to integrated care (Kodner and Spreeuwenberg 2002). However, integrated care itself is fraught with further challenges due to misaligned incentives and lack of trusting relationships among members. Hence integrated care provides a suitable context to study ecosystem evolution. 
Specifically, we examined an integrated care ecosystem formed between an acute hospital (the keystone organization) and several partner nursing homes (members) to provide specialist care to elderly patients in nursing homes via telemedicine. Telemedicine "involves the exchange of medical information from one site to another via electronic communications to improve a patient's clinical health status" (Yeow and Goh 2015). Telemedicine hence allowed for a joint medical assessment where the patient's medical information is shared between the nursing homes and specialists over technology. We collected archival data and conducted 29 semi-structured interviews with both acute hospital and nursing homes. This data was then extensively analyzed by identifying key tensions and orchestration actions from the data and then iterating this with the literature.

Consistent with prior research, our longitudinal analysis revealed three broad phases of evolution- member enrollment, joint capabilities development and ecosystem renewal. We then identified three tensions most salient in each phase of evolution: individual vs collective identifications, actual vs expected capabilities and existing vs new commitments. More importantly, our findings further revealed the additional orchestration actions taken by the keystone organization to specifically manage these tensions which we label as adaptive orchestration actions - Identity adaptation, Capability adaptation and Boundary adaptation.

This study builds and extends prior research on ecosystem evolution and orchestration in many important ways. First, our process model explicitly highlights the evolution trajectory of an ecosystem along with the tensions emerging in each phase. This reconciles prior literature where the two aspects of evolution and tensions have been dealt with separately. Our findings suggest that specific tensions impact the ecosystem evolution in specific phases over time. Second, while prior studies have mostly assessed tensions in innovation and goods dominant ecosystems (Garud et al. 2002; Wareham et al. 2014), our findings reveal two tensions which occur especially in a service ecosystem- actual vs 
expected capabilities and existing vs new commitments. Third, we develop the concept of adaptive orchestration from our findings, distinguishing them from the more routine actions identified from the literature (Batterink et al. 2010; Dhanaraj and Parkhe 2006; Nambisan and Sawhney 2011; Prince et al. 2014). Adaptive orchestration actions differ from routine orchestration actions as these take into account member feedback and are emergent in response to the tensions whereas routine actions are initiated and driven by the keystone organization alone to facilitate evolution. In other words, adaptive orchestration actions complement the routine actions as they address the specific tensions as and when they arise during ecosystem evolution.

While Study 1 attempts to explicate tensions and the keystone organization's actions to manage them, Study 2 explores the second critical aspect of ecosystems - value derived by members from ecosystems. Recent research in ecosystems has started to explore the value members derive from participation in an ecosystem (Ceccagnoli et al. 2012; Han et al. 2012). However, there are important aspects that remain relatively unexamined.

First, prior research has examined value to members measured as changes in the market value (in form of abnormal stock returns or sales) when they associate themselves with an ecosystem (Ceccagnoli et al. 2012; Han et al. 2012). While this is a significant aspect of value to members, value in the form of member's financial performance (revenue or profits) or non-economic value (quality of service) still remains relatively understudied (Han et al. 2012; Sherer 2014). This partial assessment of value often leads to an underestimation of value that members derive from participation in ecosystems. We also believe this holds important consideration for members on their decision to join and remain associated with ecosystems. One motivation for members to join ecosystems is to create value along with the keystone organization that they cannot create on their own. If they are not able to benefit from partnership over time, they may leave and join other ecosystems. It is hence important 
to examine the value that members derive from their participation over time and not just from one-time association with the ecosystem.

Second, prior research suggests that value co-creation in ecosystems requires exchanges of critical resources and services and development of symbiotic relationships among the members and the keystone organization (Grover and Kohli 2012; Lusch and Nambisan 2015; Sarker et al. 2012). Members hence derive value from the ecosystems by participating in these exchanges over time. Value is seen unfolding and emerging with time through these exchanges. While studies have clearly indicated the importance of these value co-creational exchanges, yet how these result in value to members remains unexplored (Grover and Kohli 2012). We believe this holds significant importance since ability of an ecosystem to sustain members is dependent on the value it continues to generate jointly with the members. In the second essay, therefore we address the following research question

\section{RQ2: How do value-cocreation exchanges among the members and the keystone}

\section{organization influence the value to members over time?}

To study this research question, we adopt a quantitative approach of panel data analysis with fixed effects to observe the value to members over time in an integrated care ecosystem. Theoretically, we conceptualize the integrated care ecosystem as a service ecosystem where value is co-created through collaborative exchanges of services (or application of competencies) among diverse healthcare providers. Drawing on this service view and broader value co-creation literature, we explicate three value co-creational exchanges which we term as - service exchange, resource exchange and relational exchange (Grover and Kohli 2012; Lusch and Nambisan 2015; Sarker et al. 2012). We then propose a model for value co-creational exchanges and value to members measured in both economic (cost effectiveness) and non-economic forms (user satisfaction and clinical outcomes). 
We examine this question in context of a healthcare service ecosystem which was formed between an acute hospital and four partner nursing homes to provide specialist consultations to the elderly patients in nursing homes via telemedicine. We constructed panel dataset by collecting archival data from 2010 to 2015 for all four nursing homes. This data was then analyzed using two-stage least squares with fixed effects.

The pattern of findings revealed from our data analysis suggests important considerations for members in a service ecosystem. Our findings revealed that all three value co-creational exchanges are critical to the value members derive from ecosystem, albeit differently. While resource exchanges improved the clinical outcomes, service exchanges improved the user satisfaction and cost effectiveness of the members. Moreover, relational exchanges improved the willingness of members to engage in more resource exchanges. While prior research has noted the importance of resource exchanges in value co-creation, our findings suggest that although resource exchanges were necessary, they were not sufficient to provide the entire range of value to members. In fact, members accrued greater range of benefits when they were involved in service exchanges. Hence, participation in different value co-creational exchanges, can bring a wider range of value to members (both economic and non-economic).

In summary, both Study 1 and Study 2 together contribute to the literature on ecosystem evolution and value co-creation and have implications for both members and the keystone organization since both tensions and lack of value to members undermine the sustainability of the ecosystem. Hence, while the keystone organizations need to be vigilant in managing tensions, members also need to understand how their participation effects the value they derive from ecosystems over time. Together, this mixed methods investigation and perspectives from both the keystone organization and the members provides a holistic examination of both evolution as well as value co-creation in ecosystems. 
The rest of this dissertation is organized as follows. In Chapter 2 the first research question of how the keystone organization manages the evolution of the ecosystem while addressing the salient tensions in each phase is explored. Chapter 3 investigates the second research question of how value co-creational exchanges among the keystone organization and its members influence the value to members over time. Contributions and implications of are findings are also discussed in each chapter. Chapter 4 integrates and provides a summary of findings from both the studies and suggests avenues for furthering research. 


\section{CHAPTER 2.}

\section{TENSIONS IN ECOSYSTEM EVOLUTION: EVIDENCE FROM INTEGRATED CARE}

\section{INTRODUCTION}

Organizations today are increasingly developing ecosystems to avert the threat of dying in silos or to generate more value by leveraging capabilities of other members (Adner 2006). A business ecosystem is a "community of interacting entities - organizations and individuals (including customers) that coevolve their capabilities and roles and depend on one another for their overall effectiveness and survival" (Iansiti and Levien 2004; Lusch and Nambisan 2015, pp. 161; Moore 1993). Together, members of an ecosystem share symbiotic relationships and work collaboratively to deliver a plethora of complementary products and services (Kandiah and Gossain 1998). These members are often led by a keystone organization that forges partnerships, develops necessary capabilities and ensures the ecosystem's continued survival (Iansiti and Levien 2004; Jha et al. 2016; Moore 1993; Srivastava and Shainesh 2015; Tan et al. 2015).

Ecosystems themselves are also subjected to both external and internal challenges, which if not addressed, may cause them to collapse (Reeves et al. 2016). For example, changes in the technological landscape may result in the rise of competing ecosystems. This is illustrated by the decline of Eastman Kodak's traditional analog printing business with the rise of digital imaging. Although Kodak had developed an ecosystem that comprised of a wide range of manufacturers, film sellers and retail partners to support film based photography, the growth of digital imaging diverted many retailers to Fuji's rising ecosystem. Similarly, during 2000-2010 Symbian's ecosystem was the most popular smartphone 
operating system platform, shipping nearly 450 million mobile phones. However, the rise of competing ecosystems of Android and iOS eventually led to the extinction of the Symbian ecosystem as the keystone organization struggled hard to respond to the changes (West and Wood 2013). There are also internal threats to an ecosystem. A member with weaker capabilities or recurring bottlenecks due to differences in members' organization cultures and incentives may reduce the amount of value co-created by the ecosystem (Adner and Kapoor 2010; Garud et al. 2002; Iansiti and Levien 2004; Lindgren et al. 2015; Wareham et al. 2014).

Ecosystems must hence evolve in order to address these external and internal challenges. Analogous to a biological ecosystem, ecosystems evolve through mutual and reciprocal actions of the organizations in the ecosystem. These organizations share high dependencies for both effectiveness and survival (Iansiti and Levien 2004) and hence coevolve together as they respond to the changes in internal and external environment (Kim et al. 2010). With this, the ecosystem as a whole, evolves over time. While this evolution results in changes in depth and breadth of relationships and resources and services offered by the ecosystem (Kandiah and Gossain 1998; Paul 2006; Peltoniemi 2006; Srivastava and Shainesh 2015; Tan et al. 2015; Zeng 2015), this does not always result in ecosystem survival. Clearly, not all ecosystems evolve swiftly to resolve challenges or continue to move in the right direction, which is why they fail to survive. Often the keystone organization plays a critical role in building the direction and pace of evolution. Hence, it becomes imperative for the keystone organization to also address these challenges or tensions as they arise.

Recent literature has started to explore the ecosystem evolution and the role of the keystone organization (Jha et al. 2016; Leong et al. 2016; Srivastava and Shainesh 2015; Tan et al. 2015). These studies have identified phases of ecosystem evolution and suggested a range of orchestration actions taken by the keystone organization to lead this evolution. Another recent stream of research has started to examine the tensions that impact the 
ecosystem evolution (Garud et al. 2002; Lindgren et al. 2015; Wareham et al. 2014). Tensions refer to the competing demands in the ecosystem (Smith and Lewis 2011). For example, ERP ecosystems often face the competing demands of standards vs variety in technological components. However, most of these studies identify and study tensions at a specific point in time rather than taking a longitudinal perspective of how tensions unfold together with the evolution. The latter has stronger implications on the role of the keystone organization, since the keystone organization has to address these tensions as they emerge in each phase and then facilitate the ecosystem evolution.

In light of these gaps, our research question therefore is how does a keystone organization manage the evolution of ecosystem and address the emergent tensions? We examine this question in the context of healthcare which has seen the growth of ecosystems developed to provide cost effectiveness and improved continuity in care (Rajan et al. 2013; Singh et al. 2011; Srivastava and Shainesh 2015). Specifically, we examine an integrated care ecosystem formed between an acute hospital (the keystone organization) and several partner nursing homes (members). This ecosystem was formed to provide specialist care to elderly patients in nursing homes via telemedicine.

Through our longitudinal case analysis, we first identify the salient tensions in each phase of evolution and then conceptualize a set of orchestration actions which we label as 'adaptive orchestration actions' that the keystone organization enacted to manage the tensions. In addition, we propose a process model of evolution which encompasses a set of routine orchestration actions identified from prior literature together with the tensions and adaptive orchestration actions surfaced by our case.

In what follows, we first provide a review of the literature on ecosystem evolution, tensions and keystone orchestration actions. In the later sections, we describe our research methodology, followed by findings, discussion, contributions implications and conclusion. 


\section{BUSINESS ECOSYSTEMS}

In this section, we first briefly describe the characteristics of business ecosystems and how they evolve over time. We then focus on the role of the keystone organization in the orchestration of the ecosystem followed by the tensions faced by the ecosystem.

\section{Business ecosystem Characteristics}

Business ecosystems are characterized by a diverse set of members that are held together by symbiotic relationships and a shared purpose. Ecosystems extend beyond the traditional supply chain and may include competitors, customers and regulators such as government agencies (Iansiti and Levien 2004). Members in an ecosystem differ in terms of their cultures and business processes, roles and capabilities (Lusch and Nambisan 2015). These members however, are linked by symbiotic relationships as a member's performance is often dependent on the resources and capabilities of other members (Adner and Kapoor 2010; Iansiti and Levien 2004). Members hence work together by defining a shared purpose (mission and vision) which guides their activities over time (Lusch and Nambisan 2015).

However, challenges in the ecosystem often stem from this very diversity and interdependence as the ecosystem success is dependent on the member's ability to jointly deliver services (Adner 2006). Member actions therefore are usually orchestrated by a keystone organization which acts as a crucial hub in the ecosystem. The keystone organization facilitates the joint creation of value in the form of products and services (Kandiah and Gossain 1998). It also shares the value created with the members by investing in assets such as platforms and technology which act as a foundation for exchange (Cusumano and Gawer 2002; Tiwana et al. 2010). Further, the keystone organization also establishes governance to set rules for participation and resource sharing, to monitor fair distribution of value and to resolve conflicts if any in the ecosystem (Robertson and Choi 2010). 
However, it is important to note that ecosystem characteristics of shared purpose, symbiotic relationships, platforms and ecosystem governance are not developed overnight, but are nurtured over time in the ecosystem (Moore 1993; Peltoniemi 2006). Hence the keystone organization plays an important role in the evolution of the ecosystem.

\section{Business ecosystem evolution and role of the keystone organization}

Ecosystems evolve over time (Moore 1993). This evolution is triggered either from changes in internal decisions, motives and feedback of members, or changes in external environment (government regulations or customer preferences) which result in changes in depth and breadth of relationships, resources and services offered in the ecosystem (Kandiah and Gossain 1998; Paul 2006; Peltoniemi 2006; Srivastava and Shainesh 2015; Tan et al. 2015; Zeng 2015). The ecosystem responds to the evolution through mutual and reciprocal actions among the members, often led by the keystone organization (Iansiti and Levien 2004). The keystone organization engages in a set of orchestration actions defined as a "set of deliberate, purposeful actions undertaken to create value and extract value" (Dhanaraj and Parkhe 2006) to manage the evolution of the ecosystem.

Recent work in ecosystem has started to explore this evolution by conducting longitudinal studies (Jha et al. 2016; Leong et al. 2016; Srivastava and Shainesh 2015; Tan et al. 2015; Zeng 2015). These studies have examined evolution in different contexts such as healthcare (Srivastava and Shainesh 2015) and e-commerce (Jha et al. 2016; Tan et al. 2015). The focus has been on identifying different phases of evolution and the role of the keystone organization in the orchestration of the ecosystem. Our review suggests three broad phases of ecosystem evolution- member enrollment, joint capabilities development and ecosystem renewal (Jha et al. 2016; Leong et al. 2016; Srivastava and Shainesh 2015; Tan et al. 2015). See Table 1 
The member enrollment phase of the ecosystem refers to the genesis of the ecosystem where the idea for the ecosystem is conceptualized and potential partners are identified (Jha et al. 2016; Srivastava and Shainesh 2015; Tan et al. 2015). The keystone organization hence engages in two interrelated orchestration actions- assessing demand and developing the value proposition to potential members, and seeking potential partners (Jha et al. 2016; Srivastava and Shainesh 2015; Tan et al. 2015). For this, existing resources such as knowledge (Srivastava and Shainesh 2015) and capabilities such as market responsiveness and technical capabilities (Tan et al. 2015) are leveraged by the keystone organization to assess member needs and explore possible solutions. The keystone organization then reaches out to potential partners and starts building trusting relationships (Jha et al. 2016; Nambisan and Sawhney 2011). The keystone organization defines a shared purpose for the ecosystem by clearly charting the mission and vision statements (Robertson and Choi 2010).

For example, Tan et al (2015) in their study on Alibaba's ecosystem note that in the first phase, Alibaba (the keystone organization in this case) realized that in the e-commerce market, SMEs lacked the capabilities to go online as internet was not very prevalent at that time. Hence, through its market responsiveness and technical capabilities, Alibaba opened its platform and developed a B2B marketplace with a trusted online escrow service for merchants and buyers. Thus by mitigating the fears of online transactions and easing the process to participate online, Alibaba provided a unique value proposition for members to join its platform.

The joint capabilities development phase refers to the creation of operational infrastructure such as a technology platform to jointly deliver ecosystem services (Cusumano and Gawer 2002; Leong et al. 2016; Srivastava and Shainesh 2015; Tan et al. 2015). The keystone organization actions in this phase are focused on developing technology platform and resources for exchange and then establishing the joint processes. For this, the keystone 
organization invests in developing necessary resources such as technology (Srivastava and Shainesh 2015) \& knowledge and related processes such as deploying formal or informal forums to share knowledge (Dhanaraj and Parkhe 2006; Dyer and Nobeoka 2000; Zahra and Nambisan 2012). The keystone organization also ensures fairness and transparency for all members (Zahra and Nambisan 2012) through shared decision making to encourage participation.

For example, Jha et al, (2016) in their study of the evolution of an agricultural ecosystem suggest that after the member enrollment, the keystone organization started to focus on developing the technology platform for soil nutrient analysis. To encourage the use of the platform, the keystone organization then trained the members on the use of the technology and provided backup support. It also established farmer interest groups to increase interaction among members, share farming activities and jointly solve problems. By doing this, the keystone organization was able to establish a platform to jointly deliver value.

The ecosystem renewal phase refers to the continued growth of the ecosystem to increase its resilience against internal and external changes in the environment and prevent it from dying (Leong et al. 2016; Moore 1993). Once the platform starts delivering services, in this phase, the keystone organization is focused on diversification of ecosystem services and monitoring the ecosystem performance to identify internal weaknesses (Dhanaraj and Parkhe 2006; Gausdal and Nilsen 2011; Leong et al. 2016; Moore 1993). For this, the keystone organization may use institutional resources (Srivastava and Shainesh 2015) and leadership capabilities (Tan et al. 2015) to manage the extended diversity of the ecosystem.

For example, Leong \& Pan, (2016) in their study of evolution of a rural e-commerce ecosystem suggest that once e-commerce was established with an operational infrastructure to deliver agricultural products, in the ecosystem renewal phase, more retailers joined and started using the platform to sell diversified products such as stamps, shoe insoles, kids wear, 
flower tea to promote rural development. Increase in product offerings provided resilience from unexpected changes in internal and external environment. In addition, complementary industries such as infrastructure, and telecommunication also gained ground and provided better internet facilities making the ecosystem more self-reliant.

Put together, these studies suggest that ecosystems evolve through three phases of evolution- member enrollment, joint capabilities development and ecosystem renewal. Table 1 summarizes the keystone organization's actions in each phase of evolution. It is important to note here that each phase is distinct in terms of its objective and related orchestration actions. However, the ecosystem evolution through these three phases is unlikely to unfold without challenges. As ecosystems evolve, they are subjected to internal and external environmental challenges which, if not addressed, may cause the ecosystem to collapse. For example, an ecosystem may face difficulties in attracting members to its value proposition due to lack of trust (Jha et al. 2016) or it may be challenged by competing ecosystems and experience loss of members to other ecosystems (Moore 1993; Reeves et al. 2016). These challenges are also likely to influence the keystone organization's actions in the evolution of the ecosystem. To surface these challenges, we now move to another cluster of studies on ecosystems (Banoun et al. 2016; Garud et al. 2002; Lindgren et al. 2015; Wareham et al. 2014) that are specifically aimed to identify ecosystem tensions.

Table 1. Ecosystem Evolution and keystone orchestration actions

\begin{tabular}{|l|l|l|}
\hline Phases & \multicolumn{1}{|c|}{ Keystone Organization Actions } & \multicolumn{1}{c|}{ References } \\
\hline $\begin{array}{l}\text { Member } \\
\text { enrollment }\end{array}$ & $\begin{array}{l}\text { Assessing demand \& developing value } \\
\text { proposition } \\
\text { Seeking potential partners }\end{array}$ & $\begin{array}{l}\text { (Jha et al. 2016; Srivastava } \\
\text { and Shainesh 2015; Tan et al. } \\
\text { 2015) }\end{array}$ \\
\hline $\begin{array}{l}\text { Joint } \\
\text { capabilities } \\
\text { development }\end{array}$ & $\begin{array}{l}\text { Developing technology platform and } \\
\text { resources }\end{array}$ & $\begin{array}{l}\text { (Cusumano and Gawer 2002; } \\
\text { Leong et al. 2016; Srivastava } \\
\text { and Shainesh 2015; Tan et al. } \\
\text { 2015) }\end{array}$ \\
\hline $\begin{array}{l}\text { Ecosystem } \\
\text { renewal }\end{array}$ & $-\begin{array}{l}\text { Diversification of ecosystem services } \\
\text { Monitoring ecosystem performance }\end{array}$ & $\begin{array}{l}\text { (Dhanaraj and Parkhe 2006; } \\
\text { Gausdal and Nilsen 2011; }\end{array}$ \\
\hline
\end{tabular}




\begin{tabular}{|l|l|l|}
\hline & & $\begin{array}{l}\text { Leong et al. 2016; Moore } \\
1993)\end{array}$ \\
\hline
\end{tabular}

\section{Ecosystem Tensions}

We use the term tension in this study following (Garud et al. 2002; Lewis 2000) and (Banoun et al. 2016) to highlight the challenges or competing demands in the ecosystem. Tensions are defined as simultaneously existing competing demands or disagreement among the actors in an ecosystem (Banoun et al. 2016; Smith and Lewis 2011). While studies have labelled these tensions differently, these tensions often arise from either the differences in identities and expectations of members (Banoun et al. 2016; Lindgren et al. 2015) or the differing objectives in the use of the technology platform (Garud et al. 2002; Wareham et al. 2014). These tensions can be classified under three broad categories- individual vs collective identifications, standard vs variety, and autonomy vs control (Wareham et al. 2014).

The first tension is that of individual vs collective identifications. This tension highlights the competing demands between the existing identities and incentives of individual organizations and their constant need to be aligned to a new collective ecosystem identity and focus on the good of the ecosystem as a whole. For example, Lindgren et al (2015) explicate the identity aspect of this tension in the context of a mobile ecosystem which was formed among public and private organizations to deliver traffic information services. The collective identity of the ecosystem was aimed to provide digital traffic services by developing innovation and technology capabilities. However, the legitimation of this new collective identity entailed configuring of existing relationships with other members such as technology vendors and car manufacturers which had disparate mission and goals. As the keystone organization (public administrator of roads) initiated creation of this new collective identity it resulted in identity tension as it raised questions about the beliefs of the keystone organization itself which was not a service provider and rather an administrator of 
maintaining physical infrastructure and roads. These ambiguities on the role and beliefs of the keystone organization resulted in lack of cooperation from private organizations as they struggled with what the ecosystem stands for. While Lindgren et al (2015) focused on the differences in identities, in another study, Wareham et al (2015) took the context of ERP ecosystem to highlight the incentives aspect of this tension where profit seeking behaviour of members affected the greater collective needs of the ecosystem. In this case, the provider of an ERP software suite developed an ecosystem with third party development and solution partners to provide holistic and customized software to small medium enterprises. On one hand, these partners were to contribute to innovative solutions for the ecosystem and on the other hand these were also motivated towards their individual benefits like financial compensation. This disparity in individual and collective incentives required the keystone organization to establish incentives to promote both complementary applications as well as entrepreneurial responses to client needs. The keystone organization hence established governance mechanisms such as best practices, collaboration templates and changes in process definitions to balance the tension.

Another tension is that of standards vs variety. This tension highlights the need to increase innovation through exploration while maintaining the standards of the platform. While technology platforms are designed to increase innovations, a loose coupling between core and complement components of the platform increases fragmentation and tight coupling stifles innovation (Wareham et al. 2014). For example, in the context of an ERP ecosystem, Wareham et al (2014) suggest that having open source code or ease of customization increases the risk of fragmentation, however developing mechanisms such as verification of module compatibility or standard certifications for partners can help to constrain it.

This also raises the related tension of control vs autonomy which is reflected in the quality of services produced by the platform. The autonomy given to the members to produce 
innovations has to be balanced with control over the production quality of complements. For example, this tension is acknowledged by Garud et al (2002), albeit labelled differently, where they examine how Sun Microsystems (the keystone organization) sponsored Java to be the standard platform in the emerging ecosystem. Sun Microsystems had to balance the autonomy with control among the members in a way that ensured Java's evolution while avoiding the creation of incompatible versions by members. Again, standards had to be introduced to restrict the variety in components developed by the members.

Along with tensions, these studies have also suggested skills, and orchestration actions required to manage these tensions. For example, Garud et al (2002) suggest that to manage the tension of standards vs variety, the keystone organization should possess social and political skills. Social skills are required to instill cooperation among members and develop a standardized use of platforms. Political skills on the other hand are required to manage situations when members may deviate from the standards.

Other studies recommend a range of actions that the keystone can take. Banoun et al (2016) suggest three types of actions to manage tensions in general - clarification, local arrangement and compromise. Clarification involves finding a common purpose to resolve conflict. Local arrangement refers to an explicit arrangement between members after initial negotiation and compromise involves more durable agreement between members focused on the commonly derived benefit.

Lindgren et al (2015) and Wareham et al (2014) on the other hand, suggest some orchestration actions taken by the keystone organization to manage specific tensions. Lindgren et al (2015) in their study of traffic ecosystem development suggest that to manage the tension of individual-collective identifications the keystone organization had to redefine its role from just being a transport authority to a more powerful innovator and digital services provider. For this, new goals were developed around traffic safety, sustainability and 
efficiency. The keystone organization also laid down rules for ownership of traffic data and service delivery conditions. Wareham et al (2014) suggest that to manage the tension of standard vs variety, the keystone organization introduced governance mechanisms such as problem solving, technical support and certification of partner solutions to reduce variance in processes and outputs associated with the ERP platform.

While these studies (Garud et al. 2002; Lindgren et al. 2015; Wareham et al. 2014) have suggested that tensions exist in the ecosystem and that the keystone organization plays an important role in managing these tensions, it is still not clear if tensions only occur at specific points of ecosystem development or if there are different salient tensions at each phase of ecosystem evolution. Recent work by Banoun et al (2016) addresses this issue in part by suggesting that evolution of an ecosystem alternates between phases of tensions and solutions after which stability is achieved in the ecosystem. They study the evolution of a service ecosystem after the introduction of a platform. They particularly focus on the individual-collective identifications tension which arises due to different expectations of members over time. For example, in the first phase, tension arose as some members were more focused on the technical aspects while others on the traditional procedures. The differences in expectations affected the joint delivery of ecosystem services. Hence the relationships were clarified through communication and standardization of work procedures was increased. However, in the next phase, tension arose again as now members felt the standard industrial procedures were too rigid and lacked market orientation affecting adversely their clients. Hence again temporary changes were introduced to meet client expectations resulting in evolution of the ecosystem.

While prior literature has suggested that tensions exist in the ecosystem and also that keystone organization plays an important role in the evolution of the ecosystem, less is known about the ongoing relationship between tensions and evolution. The broader literature 
on tensions suggests that the keystone organization's responses to tensions shapes the evolution of the ecosystem and its eventual destiny (Smith and Lewis 2011). For example, during the inception, the ecosystem faces the tension of individual vs collective identifications due to competing incentives and identities of members. The keystone organization often engages in the orchestration action of forging a new goal for the ecosystem. This leads the ecosystem to a new phase where members collectively develop capabilities to meet the new shared goals. This suggests that while tensions stem from inherent ecosystem characteristics and changes in environmental context, when addressed by the keystone organization, these result in evolution of the ecosystem.

In sum, these studies suggest that tensions exist during the ecosystem evolution. The studies also suggest that the keystone organization plays an important role in managing these tensions. However, because the handful of studies on tensions were either cross sectional, or focusing on one or two specific tensions, little is known about whether tensions evolve over time, or whether different tensions arise at different points of ecosystem evolution. Further, relatively few studies (Banoun et al. 2016; Garud et al. 2002; Lindgren et al. 2015) examine the important issue of how the keystone organization can manage these tensions over time and how it results in the evolution of the ecosystem.

Given this, our research question is how does the keystone organization, through its orchestration actions, address the emergent and ongoing tensions along with the evolution of the ecosystem? We explore this question in the context of an integrated care tele-geriatrics ecosystem, formed between an acute hospital and several nursing homes to deliver specialist services to the elderly patients. In the next section, we present our methods followed by findings and discussion. 


\section{METHODS}

We adopted a case study methodology to study our research question. Case studies are a useful tool to investigate phenomenon which are complex or less researched (Dubé and Paré 2003; Yin 1994). Since the question in context required an in-depth investigation to surface tensions and related keystone organization actions over time, we chose a revelatory case study approach as it enabled us to collect and analyze a rich dataset over time (Yin 2003).

\section{Case Selection}

We selected a case that was theoretically relevant to our research question (Dubé and Paré 2003) - an ecosystem orchestrated by a keystone organization. We selected an acute hospital (Acute North) and its ecosystem of four nursing homes as the case site for following reasons.

First, Acute North, the keystone organization, played a vital role in the orchestration of the tele-geriatrics ecosystem with nursing homes as partners. Acute North was designated as an anchor hospital for other providers in the north region. However, since providers in the region were very disparate and shared arm's-length relationships, Acute North had to orchestrate the development of ecosystem from scratch. This hence provided us an opportunity to observe Acute North's actions more closely in developing the ecosystem.

Second, the context of integrated care suggested that tensions would arise from the diversity. While Acute North was a well-funded public hospital with state-of-the-art facilities, nursing homes were run by private religious welfare organizations with lower levels of resources. These nursing homes worked autonomously and maintained arm's-length relationships with acute hospitals which were considered instruments of the government.

Third, the tele-geriatrics ecosystem had successfully evolved over a period of five years and is now an operational ecosystem. This is in contrast with many studies in integrated 
care that have been pilot projects ranging six months to a year, with many cases of unsuccessful initiations. This hence provided a longitudinal context to study emerging tensions, orchestration actions over time, as well as the evolution of a relatively successful ecosystem.

Finally, we had access to both Acute North and nursing homes' perspectives to the ecosystem evolution process, the tensions faced, and the keystone organization's actions towards those tensions. This enabled us to obtain a rich and detailed account of events over time from different perspectives.

\section{Context: Integrated Care}

Healthcare today is facing formidable challenges of managing a rapidly ageing population, rising demands and costs of the services. This has increased the need for specialized services, which in turn has caused the delivery of care to become fragmented (Kodner and Spreeuwenberg 2002; Stange 2009; Stille et al. 2005) resulting in a lack of consistency and continuity in care (Kodner and Spreeuwenberg 2002). One frequently recommended solution for addressing these challenges is Integrated Care which aims to provide healthcare under a collaborative model. For instance, a chronic patient with multiple health needs may frequently need access to both specialists and general physicians. Since care provided by different providers is interdependent, integrated care is designed to manage such varying patient needs by providing better connectivity and alignment among different providers. This coordination often involves use of various information systems such as electronic health records (for sharing patient information), remote patient monitoring (for collecting patient statistics remotely) and telemedicine (for delivering care over video conferencing) (Beyer et al. 2004; Rajan et al. 2013; Singh et al. 2011).

One of the most widely used integrated care collaboration involves the use of telemedicine to provide specialist care. A recent report by Deloitte (2015) suggests that the 
global telemedicine market which was USD 14.2 billion in 2012 is expected to grow at 18.5 percent between 2012 and 2018 (Morris and Yoritomo 2015). This use of telemedicine is illustrated, for example, by Rajan et al (2013) where they study the telemedicine enabled integrated care between community hospitals (located near patients) and specialty hospitals (geographically distant from community hospitals and patients). While community hospitals were competent to provide day-to-day care, patients had difficulty in accessing specialist care. To build continuity, community hospitals opted for telemedicine as a means to help patients receive specialist care. Patients at community hospitals were hence able to access timely care and also reduce their costs of travel to distant specialist hospitals.

While integrated care makes extensive use of technology, it is nonetheless fraught with challenges such as organizational differences, misaligned incentives and lack of trusting and credible relationships among providers (Bevan and Janus 2011; Cebul et al. 2008; Leichsenring 2004; Ling et al. 2012b; Montenegro et al. 2011; Pate et al. 2010; Pike and Mongan 2014; Pomerantz et al. 2009) and hence its sustainability over time has become an ongoing concern among healthcare providers (Bevan and Janus 2011; Tsasis et al. 2012).

Integrated care provides a suitable context to study ecosystem evolution. Like ecosystems, integrated care collaborations are characterized by diverse members such as general physicians providing primary care, specialists providing focused care, home and day care centers providing social care and regulators. These members however are held together by a shared purpose which in the integrated care context is often continuity of care or efficiency of operations and resources. For example, Singh et al (2011) studied the ecosystem of tele-home health comprising nurses (skilled to provide care at patient's homes), physicians (with deeper medical expertise) and patients. This ecosystem was formed for efficient use of nurses and physicians in providing continuous care to patients at home. Second, members in integrated care collaborations share interdependent relationships and are often led by a 
keystone organization that drives other members towards the shared purpose and also invests in technologies and resources (Singh et al. 2011). Last, both ecosystems and integrated care collaborations evolve over time (Minkman 2012). For example, in their study on telemedicine, Srivastava et al (2015) suggest three phases of evolution- idea and launch of project, infancy and early growth and later growth and expansion.

\section{Data Collection}

To ensure reliability (Eisenhardt 1989) data was collected from multiple sources. Since the ecosystem had been initiated since 2010, all the archival data from 2010 to August 2014 was first collected to generate familiarity with the context. This archival data consisted of all weekly and quarterly meeting minutes, quarterly progress reports, technology updates, annual reports of nursing homes and Acute North, senior management presentations, news and research articles published by Acute North and training \& learning documents. This was a total of 227 documents comprising 1443 pages in various formats such as word documents, pdf files, excel sheets, graphical images and presentation decks.

The archival data was analyzed to create a project timeline. This timeline highlighted the major events through the years such as when the project proposal was written, funding approved, foundational technology was installed, training initiated, start of tele-consult sessions and so on. Within each of these major events, a detailed timeline was also created to highlight more granular episodes and actions such as pilot testing, vendor selection, technology assessment. All the gaps in understanding arising from this documentation were also noted separately for clarification during the interviews.

The archival data such as attendance list of meeting minutes was used to prepare an initial interview list of key people involved in the project. This list was shared with Acute North for verification and to ensure relevant people were not missed or to exclude people which have left the project or organization. With suggestions from Acute North a final 
interview list was prepared which consisted of people from both Acute North and the four participating nursing homes (NH1, NH2, NH3, NH4) as in Table 2. Given different roles of the organizations in the ecosystem, two sets of interview questions were prepared - one for Acute North and one for the nursing homes. This also helped in focusing on specific gaps with respect to each organization. Since Acute North was the keystone organization, questions focused on why it initiated the ecosystem, what were the challenges and how they were managed, how technology was implemented and what was the future vision for the ecosystem. For nursing homes, questions focused on why they joined the ecosystems, their relationship with Acute North and associated benefits and challenges from the ecosystem. Also, for each interviewee questions were tweaked depending on their role in the project. For example, For IT department the questions focused more on technology deployment, but for operations executives the questions were focused on processes and related KPIs. The interview questions served as a guide during the interview so that important issues were not missed. A more flexible approach was used during the interviews.

A total of 29 semi-structured interviews were conducted with representatives from both Acute North and nursing homes. Each interview lasted between one to two hours. For most of the interviews at least two researchers were present. Each interview was audio recorded and the researchers also took detailed notes. Post interviews, these detailed notes were shared and then a final interview transcript was prepared for each interview. Table 2 provides a detailed list of interviewees and data collected. Lastly, the researchers also attended a few ecosystem quarterly meetings to gain additional insights into the issues and interactions among the nursing homes and Acute North. 
Table 2: Interviews

\begin{tabular}{|c|c|c|c|c|c|c|}
\hline \multicolumn{7}{|c|}{\begin{tabular}{|l|} 
Interviews (Total 29) \\
\end{tabular}} \\
\hline \multicolumn{2}{|c|}{ Acute North } & Nursing Homes & NH1 & $\mathrm{NH} 2$ & NH3 & NH4 \\
\hline $\begin{array}{l}\text { Senior } \\
\text { Consultant }\end{array}$ & 1 & $\begin{array}{l}\text { Tele-geriatrics } \\
\text { Nurses }\end{array}$ & 4 & 3 & 5 & 2 \\
\hline $\begin{array}{l}\text { Senior } \\
\text { Operations } \\
\text { Manager }\end{array}$ & 1 & Nurse Manager & 1 & 1 & - & 1 \\
\hline $\begin{array}{l}\text { Operations } \\
\text { Executives }\end{array}$ & 2 & \multirow{3}{*}{$\begin{array}{l}\text { Management } \\
\text { (CEO/Executive } \\
\text { Director/Nurse } \\
\text { Director) }\end{array}$} & \multirow[t]{3}{*}{-} & \multirow[t]{3}{*}{2} & \multirow[t]{3}{*}{1} & \multirow[t]{3}{*}{1} \\
\hline $\begin{array}{l}\text { Clinical } \\
\text { Educators }\end{array}$ & 2 & & & & & \\
\hline IT team & 2 & & & & & \\
\hline Total & 8 & - & 5 & 6 & 6 & 4 \\
\hline
\end{tabular}

\section{Data Analysis}

In the first step of data analysis, phases of ecosystem evolution were identified. The timeline prepared earlier with the archival data was now used along with the insights generated from reading the interview notes to identify major events through the years. This was interpreted in the light of exiting literature on ecosystem evolution and three broad phases of ecosystem evolution were identified - member enrollment, joint capabilities development and ecosystem renewal.

Guided by the literature on ecosystems and orchestration, we then identified the key actions taken by the keystone organization in each of these three phases of the ecosystem. The literature was useful in generating a set of candidate actions usually taken by the keystone organization in an ecosystem. With this information, the interview transcripts were read several times to highlight the specific actions taken by the keystone organization in this study. For example, the literature suggested that in the member enrollment phase, the keystone organization assesses the demand and develops a value proposition (Batterink et al. 2010; Jha et al. 2016). It was noted in the interviews that Acute North initiated the idea of 
tele-geriatrics ecosystem after it faced a resource crunch due to unplanned admissions of the elderly patients from the nursing homes. This action was hence labelled as initiating the idea under orchestration actions in the first phase.

Similarly, in the second phase of developing ecosystem, the literature suggests that the keystone organization is focused on developing necessary platforms and resources (Cusumano and Gawer 2002; Zahra and Nambisan 2012). It was observed here that once the nursing homes were on the board, Acute North was focused on developing the necessary resources such as technology and knowledge and processes such as reporting and how to use the platform in the ecosystem. For example, the IT team at Acute North indicated that

"We helped in sourcing for the conferencing set. They needed to be able to support dual monitors, have I/O ports for medical devices, and ports for additional video recorder and secondary inputs. "- IT team, Acute North

For the third phase, the literature suggested that the keystone organization diversifies its services in this phase to build sustainability in the ecosystem (Dhanaraj and Parkhe 2006; Gausdal and Nilsen 2011). Guided by this, it was noted that Acute North introduced new services such as ad hoc consultations to allow quick access to specialist services. This is suggestive of the quote below.

"Staff will call me to ask if they can have ad hoc. We look for doctor who is around. I ask what the history is and findings and clinical details. This is referred to whatever doctor is around. "- Clinical Educator, Acute North

Next, we validated our analysis of phases and orchestration actions by preparing a chronological case and presenting the findings to Acute north as well as the nursing homes. These sessions were useful in validating and filling our gaps in the understanding of the case. The hospital management, nursing home administrators as well as nurses fed back that the preliminary case reflected their understanding of the events. They 
broadly agreed with the three phases and provided additional clarifications and reiterated that this development was challenging. The supplementary notes from these meetings were also added to the case analysis.

This validation was instrumental in driving our attention to the challenges that were faced by the ecosystem during the evolution. Hence, we undertook a more detailed analysis to surface the challenges or tensions faced by the ecosystem during evolution. Tensions are competing demands or disagreements faced simultaneously by the organizations in an ecosystem. For example, the literature suggests that the tension of standard vs variety is often seen in ERP ecosystems where implementing technology standards stifles innovation and allowing for variety increases fragmentation (Wareham et al. 2014). To identify such tensions, we simultaneously analyzed the keystone organization's actions and member's responses to them. For example, as noted previously, in the first phase, it was found that the Acute North initiated the idea of developing the ecosystem and then invited partnerships from the nursing homes. However, it was found that when Acute North initiated these actions, nursing homes were wary of differences in identities. While Acute North was a government funded and well resourced public hospital, nursing homes were voluntary and charitable organizations. These differences resulted in tension as Acute North and the nursing homes were unable to align to a collective identity of tele-geriatrics ecosystem. Following literature, we labelled this tension as 'individual vs collective identifications' to highlight the competing nature of the tension.

This detailed analysis of orchestration actions and tensions was then again presented to Acute North and a written case report was submitted. An important feedback from this meeting was that some of the Acute North's actions that were identified earlier, were in response to the tensions that emerged during the ecosystem evolution. For example, in the second phase when ecosystem moved into developing capabilities, tension emerged due to 
actual vs expected capabilities of the members. We found that when Acute North started developing materials for nurse training, the nursing homes found the training difficult to adopt as they found the training material to be 'too medically oriented'. This clearly displayed incongruence in the actual capabilities of members and what Acute North expected of them. This further affected the creation of ecosystem capabilities as members were not able to work together effectively. Acute North, hence started to response and adapt to member constraints and customized the course to suit the nursing home's needs.

This led us to understand that there was a conceptual difference among these orchestration actions. While some of the orchestration actions were aimed to facilitate ecosystem evolution, some were in response to the tensions that were developed in the ecosystem. In fact, the latter set of actions were found to be different from the candidate orchestration actions we identified from the literature. We differentiated and labelled these two set of orchestration actions as routine orchestration and adaptive orchestration. It was found that while routine orchestration actions were ones initiated and driven by the keystone organization alone to facilitate the development of ecosystem, adaptive orchestration actions were enacted specifically to manage the tensions and hence were suggestive of much stronger involvement of members.

In the next section we present findings from the case followed by discussion.

\section{FINDINGS}

With a rapidly ageing population and rising healthcare needs, the government of the Asian country in context recently initiated a restructuring of the healthcare sector into regional clusters to promote integrated care. Acute North was designated as the anchor hospital for the northern cluster. With almost 600 beds, Acute North was equipped with the state of the art technology and equipment, and offered a range of services such as acute and emergency care, geriatrics, cardiology, nursing and clinical support. Other providers in the 
northern cluster were step-down facilities such as nursing homes catering to the elderly and chronically ill patients; community hospitals providing intermediate healthcare (e.g. wound management), polyclinics and general practitioners providing primary care and family medicine services. These providers were hence very diverse in their areas of care, medical expertise, facilities and funding. Together, these catered to nearly 700,000 people in the north region.

Care for the elderly is an increasing area of concern. According to a report published by the country's department of statistics, the number of elders is expected to rise from $8.4 \%$ in 2005 to $18.7 \%$ in 2030 . To cater to this growing need, one important set of providers are the nursing homes. Each of these manages on average 200 elderly and chronically ill patients. These nursing homes are often charitable organizations with religious affiliations. However, due to lack of funds, these institutions are always underequipped in both manpower and permanent specialist care. Each nursing home has between 15-17 nurses, and about 40 nursing aides. But these nurses are not trained to provide diagnostics and treatments specifically for the aged. While these homes usually have a general practitioner visiting them 2 to 3 times a week, the majority of patients suffer from dementia or other chronic illnesses which requires specialist treatment. However, specialist visits are costly and hence the patients in these homes are highly dependent on acute hospitals to provide specialist services.

\section{Phase 1: Member Enrollment}

Acute North, being the anchor acute hospital for north region, provided specialist services to several nursing homes. Some of these nursing homes paid for costly specialist visits twice a month. However, since these visits were at fixed times, when nursing home residents fell ill and doctors were not available, the nursing homes rushed their residents to Acute North for emergency consultations. The contextual challenges of meeting the 
healthcare demands of their elderly residents and lack of specialized geriatric expertise within nursing homes, made them heavily reliant on acute hospitals for specialist care.

Over time, Acute North started experiencing a huge influx of patients from nursing homes. Often the patients were referred to the emergency department and then discharged within a day or two as their condition did not require hospitalization. While these unnecessary transfers were traumatic for the elderly patients, it also overstretched the resources in both nursing homes and Acute North.

\section{Routine Orchestration Actions}

To moderate this growing demand, in 2010, the then head and senior consultant of geriatrics department in Acute North, proposed the use of telemedicine to diagnose and treat patients at nursing homes. By using telemedicine, nursing homes could avoid unnecessary visits to hospitals and Acute North could relieve some burden on its constrained resources. Since nursing homes had a high density of elderly patients, they were likely to utilize the service. In addition, the senior consultant noted:

"We chose nursing homes as it's a bit safer. There diagnostics, assessment is not that acute. It's usually not a life and death kind of thing. Often, it is behavioral problems, where you need not touch the patients, you can see the patient."- Senior Consultant, Acute North

The senior consultant together with the senior operations manager prepared a proposal and submitted to the Ministry of Health. The proposal labeled as Northern SeniorCare outlined the use of telemedicine to provide collaborative clinical support to patients in nursing homes. This would be facilitated by a technology platform that would enable videoconferencing between patients at nursing homes and specialists at Acute North. The nurses would facilitate the diagnosis by being the 'hands for the doctor'. For this, nurses would be trained to conduct basic diagnosis that would be shared with the specialists at Acute North 
during consultations. This would raise the standard of care in nursing homes and also reduce the unnecessary transfers from nursing homes to the acute hospitals. Under the new collective identity, Northern SeniorCare, both nursing homes and Acute North would be equal partners in delivery of better quality of care to elderly nursing home residents through the innovation of tele-medicine. The Ministry of Health assessed the proposal and approved funds for USD $\$ 1.5$ million to be used over five years.

With funds in hand, Acute North scanned the northern region for potential nursing homes and started visiting these homes to discuss their needs. The first step was to get nursing homes interested in a formal contract where a specialist would visit nursing homes at a fixed rate of USD $\$ 220$ per hour. With this service level agreement, nursing homes would then be entitled to two free additional tele-consultation sessions a month.

\section{Tension: Individual vs Collective Identifications}

As Acute North engaged in the routine orchestration actions of bringing nursing homes to Northern SeniorCare ecosystem, it was confronted with the dominant tension arising from different individual identities and organizational histories of members and their need to align to Northern SeniorCare. Acute North's identity was of a government subsidized, highly resourced provider of acute short-term care. Nursing homes' identity on the other hand was anchored in their niche as step down facilities providing long term affordable healthcare. Many of the nursing homes that were supported by supported by voluntary (often religious-affiliated) organizations also reflected their affiliations. These nursing homes operated independently and were wary of any interference from government and acute hospitals, who were seen as representatives of the government. Even though some nursing homes were already contracting specialist services from acute hospitals, they usually maintained arm's-length distance in conducting their business. Nursing homes' lack of trust in acute hospitals had been exacerbated by the negative experiences of partnerships in the 
past. Hence acute hospitals were often perceived as instruments of interference by government. As noted by Acute North's senior operations manager,

"Many times, hospitals come in and do a bunch of things - (NHs) let them do it but the NHs have to change their processes. But the hospital pulls out and then takes everything away. From all their experience, they (NHs) tend to end up being distrustful of acute hospitals." - Senior Operations Manager

With such differences in identities and lack of trust, alignment towards the new collective identity of Northern SeniorCare which was to be one of close partnership and collaboration in delivering quality care to elderly nursing home residents, was challenging. Further, there were also differences in the incentives these organizations sought from the ecosystem. While Northern SeniorCare was aimed to reduce unplanned admissions, nursing homes looked forward to benefit their residents by providing them access to specialist care. These nursing homes had more relational approach to their residents, many of whom lived at these homes for years and hence their goal was to provide affordable long term care to their residents.

\section{Adaptive Orchestration Actions}

Acute North hence engaged in two adaptive orchestration actions- leveraging on existing relationships to address divergent individual identities and setting up ecosystem governance to build collective identity and align incentives.

Leveraging existing relationships: Since nursing homes shared a distrusting relationship with acute hospitals, Acute North decided to start with nursing homes that already had some formal arrangements with them. Some nursing homes were already contracting paid specialist services from Acute North, where a geriatrics specialist visited these nursing homes each month at fixed rate/hour. This cost was about USD220 per hour and accommodated up to four patient consultations per visit. To initiate communication, Acute North leveraged its 
senior operations manager who had previous experience of working with nursing homes. When Acute North approached NH1, this dual organizational experience was leveraged to vouch for Acute North's good intentions and allay the nursing home's fears of commitment. This was instrumental in breaking the ice and created the first partnership in the ecosystem.

"Being from a voluntary welfare organization, I leveraged this to show them (NH) that I could relate to them. I knew the dark side. Then I also vouched for doctors in Acute North showing them as true clinicians and the fact that they care."- Senior

\section{Operations manager}

With NH1 on board, Acute North identified that the previous nurse manager in NH1 was now a nursing director in $\mathrm{NH} 3$ and had experience of working with Acute North in the past. Acute North then approached her and proposed the use of technology to treat patients and training to uplift nursing skills. The nurse director at NH3 evaluated the proposal of training and free specialist consultations and readily agreed. She also persuaded the staff and management to join the tele-geriatrics ecosystem.

"I was earlier in another nursing home. I wanted to duplicate all the services we had there. They (Specialists) can see the complicated cases that the GP cannot manage. By bringing them (in through tele-consultation), we save much”. Nurse Director, NH3

Acute North then approached NH4 which was relatively well funded and contracted many services from Acute North such as pharmacy, geriatricians and lab services. By this time with two nursing homes already on board, NH4 was comfortable joining the ecosystem. This growing ecosystem also helped Acute North to build partnership with NH2 who themselves approached Acute North to join the tele-geriatrics ecosystem. Each of the partners were offered two physical visits and two complementary no-charge tele-consultations. 
Two additional nursing homes also signed up but subsequently dropped out during the training phase. One dropped out because of change in their management which resulted in lack of support for tele-geriatrics and the other dropped out since it had an existing geriatrics contract with another acute hospital and joining Northern SeniorCare ecosystem could result in a conflict of interest.

It is important to mention here that while the dominant tension was individual vs collective identity, it was also found that ecosystem faced a less salient tension arising due to existing vs new commitment from nursing homes. Joining the Northern SeniorCare ecosystem would require a new commitment of nursing resources and management time from the nursing homes. However, some of the nursing homes already had existing commitments to other ecosystems. For example, one of the nursing homes dropped out due to its commitment with eastern zone healthcare ecosystem led by a different acute hospital where it was sending its patients for specialist consultations. The nursing home felt collaboration with Northern SeniorCare would result in a conflict of interest.

Creating ecosystem governance mechanisms: While the initial tensions were addressed when Acute North leveraged its existing relationships, Acute North also desired to build an accountable and equality based partnership with nursing homes in Northern SeniorCare. Acute North realized a need for stronger formal and informal communication that would surface shared concerns and challenges of partners. Hence Acute North formed a joint working forum (tele-geriatrics workgroup) consisting of representatives from all nursing homes, doctors, clinical educators and operations team at Acute North. This forum would conduct meetings every quarter to steer the strategy and discuss the challenges pertinent to tele-consultation such as use of technology. Nursing homes extensively used these platforms to voice their concerns, learn from other nursing homes and participate in decision making. 
Acute North also displayed an attitude of equal partnership and helpfulness in their communication with nursing homes.

"They are very good... they give us better scan, resources, very nice people. When we ask for resource, they are very helpful.... very good attitude -- don't worry, I am not causing you trouble, I am here to help you.". Tele-geriatrics Nurse, NH4

Acute North also leveraged these meetings to develop a shared purpose for all the ecosystem members. For Acute North, the tele-geriatrics ecosystem was a means to reduce unnecessary burden on its resources such as beds and specialists by making nursing homes more self reliant with trained nurses and use of technology. However, for nursing homes, joining tele-geriatrics ecosystem was a means to access good specialist care and improve their nurse skills. Hence Acute North set a larger shared purpose for tele-geriatrics ecosystem, i.e. improving quality of care through innovation. By doing this, Acute North reduced the dissonance that members might have with the goals of the ecosystem, and increased their identification with the Northern SeniorCare ecosystem.

\section{Phase 2: Joint Capabilities Development}

Once, Acute North had secured funding and nursing homes had agreed to join the ecosystem, Acute North began developing the necessary capabilities (resources and processes) for conducting tele-consultation. For tele-consultations, two critical resources were identified. First, a technology platform that allowed for uninterrupted high definition video and audio streaming for the whole consultation session. Second, nurses who were clinically proficient to be able to examine the patient and relay the information to the doctor since specialists had no physical contact with the patients. For example, if the doctor required the nurse to check the patient's heartbeat or liver, nurses should be able to locate the organ, palpate or listen and convey the accurate information to the doctor. In short, nurses need to be 
the 'eyes, ears, mouth piece and hands' of the specialists. As noted by the senior consultant at Acute North,

“We don't expect them to perform like a doctor. But looking at a common rash they should tell us there is a rash. They must know where liver is and all. This is not taught in their nursing school."'-Senior Consultant, Acute North

In addition to resources, Acute North also identified the need of shared processes to enable smooth coordination between nursing homes and Acute North.

\section{Routine Orchestration Actions}

To start developing technology and clinical knowledge resources, Acute North first leveraged its IT team to assess and pilot technology-based solutions. The IT team visited nursing homes to assess their existing technology infrastructure. The IT team also conducted software based trials using skype on mobile broadband and $3 \mathrm{G}$ dongle. Meanwhile, Acute North leveraged its existing pool of doctors to craft a customized training course for nurses. This was a first in the country. The course was hence developed by adapting existing material on telemedicine from the United Kingdom and the United States. The course content was designed to focus on common medical conditions of nursing home patients such as dementia, pressure ulcers and falls. Once the course material was prepared, Acute North scheduled four-hour training sessions per week in liaison with nursing homes.

Acute North also established new shared processes of tele-consultation and reporting. The tele-consultation process was divided into three parts- pre-consult, actual consult and post-consult. In the pre-consult process, the nursing homes decided the cases that need to be seen by the specialists over tele-consultation session. The clinical information like blood pressure and temperature had also to be shared prior to the consult. Acute North also assigned several members from its operations team to coordinate with the nursing homes. Nursing homes also tested the technology equipment prior to the consult and any issues were sent to 
the IT team. In the actual consult which lasted for about 15 minutes, only specialist doctor, patient and 1-2 tele-geriatrics trained nurses were required to be present. If needed, clinical educators could be involved to support the nurses. However, Acute North encouraged independent handling of cases by the nurses. In the post consultation process, the session was rated by all the participants whether it was ideal or not. An ideal session was defined as one with no issues related to technology or process. Nursing homes then shared the patient case notes and submitted it to Acute North which then saved a digital copy. Any medicines prescribed by the doctor, could be brought by nursing homes from the pharmacy at Acute North.

In addition, Acute North also established a reporting process to capture key indicators such as number of hospitalizations, consults and trained nurses. Since the tele-geriatrics ecosystem was supported by the government grant, Acute North was required to submit key performance indicators on yearly basis. Hence Acute North established a baseline for each nursing home depending on their resources and set expected targets for each year. Nursing homes recorded these numbers on monthly basis and then shared with Acute North who in turn submitted it to the Ministry of Health.

\section{Tension: Actual vs Expected Capabilities}

The tension in the first phase - individual vs collective identifications, was partially addressed through the adaptive orchestration of implementing collaborative governance mechanisms that enabled all members in Northern SeniorCare to craft common directions. This collaborative governance continued to help strengthen the collective identity as the members moved into the second phase of developing new joint capabilities. The collaborative governance mechanisms from the first phase continued, and allowed the nursing homes to discuss their concerns. As noted by the senior operations manager at Acute North, 
"We hold quarterly meetings with the NHs and use this platform to show what we want and ask them for feedback... we have to customize to the pace and standards of the NH"-Senior Operations Manager, Acute North

However, as Acute North initiated the routine actions of creating requisite resources and processes, another tension arose from nursing homes' actual capabilities. First, the current technology infrastructure at nursing homes did not support software-based consultations such as skype due to limited connectivity. This resulted in dropping of video frames and loss of audio while conducting a video-conference. When Acute North assessed the situation, they found that it was a design issue very specific to nursing home buildings which had unusually thick walls which were restricting the signals.

"We also noticed that the place...walls especially thick, and normally where people put routers...on top they have metal beams. So it's like metal beams is all over the place...So it really restricts the signal."- IT Team, Acute North

Second, the training was very difficult for nursing homes to comprehend. These nursing homes had low clinical proficiency and had not specifically received any training in specialist care. While the training course had been simplified and developed using standard materials, nursing homes still found it to be too medically oriented. Further, staff at nursing homes were not native English speakers so found it difficult to follow the training. They also felt that four hours was too demanding, as they were tired after completing their nursing duties.

"It was like reviewing the system again. It wasn't so easy. We have to put in a lot of practice. Putting theory into practice was very challenging. "- Nurse manager, NH1

Lastly, with tele-consultation, nursing homes felt stretched as they had to additionally collect data, prepare case notes and share it with Acute North after each consult. Nursing homes already had limited staff to attend to the elderly patients. This additional work over- 
extended the nursing staff as they had to collect data in standard formats and share with Acute North.

These tensions, in part, also arose from the environmental context. With high manpower shortage in the country's healthcare sector, the nursing homes relied on international nurses who were not specifically trained for geriatrics. These nurses were less proficient in English, the language used for training and by the specialists during the consults.

\section{Adaptive Orchestration Actions}

Acute North hence engaged in two adaptive orchestration actions to reduce the tension of expected vs actual capabilities - customizing technology and knowledge resources and simplifying shared processes.

Customizing technology and knowledge resources: Acute North's assessment of technology had revealed that both wireless networks which suffered from signal loss, and mobile broadband where upload speeds were slow were not reliable solutions. For a good quality audio and video conference, internet connectivity of at least $512 \mathrm{kbps}$ was desired. Acute North hence contacted various telecom vendors and assessed their options. They then opted for laying a cable network in all nursing homes as it guaranteed an uninterrupted speed of 2 mbps.

"We need very good video and good sound that can capture silent speakers."- IT

\section{Team, Acute North}

However, nursing homes fed back that they had difficulty to operate laptops during pilot testing and they lacked IT skills suggesting a need for a dedicated and simplified system. The systems should allow multitasking (nurses can share documents along with the video conference) and also allow multiple screens (for more than one nursing home if needed). Acute North hence wrote a tender for tele-conference system and invited vendors to 
demonstrate their systems. The tenders were evaluated by Acute North by comparing the products on color, glare and video quality under insufficient light.

Thereafter, tele-conference hardware was procured from the selected vendor and installed in nursing homes. Based on the requirements of nursing homes, the teleconference system was modified so that it was as easy as turning on a TV set. The training was provided by the vendor and Acute North managed the backend processes. The maintenance was outsourced to vendors and a yearly review contract was signed for replacement of faulty equipment within two days. Acute North also plugged additional devices such as hand held cameras and electronic stethoscope for better care. A multi-call unit was also installed to allow more than one nursing home to communicate simultaneously with Acute North.

While this equipment was placed in a dedicated tele-conference room in nursing homes, nursing homes soon found that it was difficult for bedbound patients to be moved to the room for consultation. Hence, the system was adapted further to be mobile. Acute North's operations team fashioned a trolley and mounted the tele-conference equipment on it. However, the initial design was less than ideal, with wires protruding, and nursing homes finding it clumsy to move. The system was then refined, adding drawers which could hold the accessories, and tidying away the wires.

While technology was being customized, Acute North also worked towards restructuring the training course to make it more practical and nursing oriented. For this, Acute North hired clinical educators who would formally develop the course. The training was redesigned into four components- identify patient symptoms, diagnose problems through clinical photographs, develop communication skills with doctors, and learn nursing interventions. As nursing homes found it difficult to lend staff for four hours of dedicated training, the training sessions were also split into two 2-hour sessions per week and were scheduled according to nursing homes' availability. 
“They try to work into the NH nurses' schedule and try not to saturate them. We go down to NH for training. We ask them about their time availability"- Clinical Educators, Acute North

Simplifying shared processes: Nursing homes also felt burdened by the shared processes created for tele-consultation. Based on their feedback, Acute North positioned its operations team to work more closely with nursing homes. The operations team helped nursing homes with coordination of consults, scheduling of nurse training, collecting feedback and reporting of data. For example, after gaining feedback from nursing homes for the pre-consult process, Acute North redesigned the information form into a simple two-page format which could be emailed, faxed or delivered in person to the operations team prior to the consult. Overall, Acute North worked with nursing homes to reduce the effort required for the shared processes. The patient selection however was entirely handled by the nursing homes as they were more familiar with the day-to-day condition of their residents.

"I prioritize the cases which need tele-consult. I look at the nature of the problem. If [general practitioner] can handle (it is ok) else I screen through." Nurse Director, NH3

During actual tele-consult process, nursing homes also raised concern that they faced difficulty due to communication issues and differences in a doctor's way of consult. Acute North hence instructed its clinical educators to handhold and provide feedback to nurses on tele-consultation process till they were able to independently handle the session.

"Nurses have different language; patient have different dialect. Sometimes doctors go a different way even if there are guidelines. Some nurses lose focus when interrupted and they have to teach the nurses to (adapt to) the specific doctor who is conducting the Tele G session."- Clinical Educators, Acute North 
Acute North also eased the process of tele-consult reporting to the Ministry of Health for nursing homes. Nursing homes found it an additional task to collect and report numbers as most of these homes did not save all their data electronically. The operations team at Acute North hence worked closely with nursing homes to help them capture data such as number of residents, number of tele consultations and unplanned admissions on monthly basis. Acute North then submitted these numbers to the ministry. As noted by the senior operations manager,

"We need to take the administration off the NHs but also ensure accountability. We have access to their admissions and emergency numbers and we report it to the ministry."-Senior Operations manager, Acute North

These adaptations to shared processes and resources, provided ground to nursing homes to level their capabilities. Hence the ecosystem was able to deliver tele-consultations services to elderly patients.

\section{Phase 3: Ecosystem Renewal}

As the ecosystem started delivering tele-geriatrics through developed capabilities, Acute North started monitoring the use of tele-consultation across nursing homes. It was observed that while nursing homes $\mathrm{NH} 1$ and $\mathrm{NH} 3$ used tele-consultations extensively, $\mathrm{NH} 2$ and NH4 had over time reduced the use of tele-consultation. This triggered further orchestration actions by Acute North to sustain the ecosystem.

\section{Routine Orchestration Actions}

Acute North started expanding the ecosystem by inclusion of new members as well as diversification of its services. First, since Acute North had been providing tele-geriatrics for more than four years now, tele-geriatrics ecosystem had a strong brand reputation among the nursing homes. This was because Acute North had always maintained fairness in partnerships with nursing homes and had always shown a helping attitude. Acute North hence invited 
partnerships from four new nursing homes (including two private nursing homes) to join the tele-geriatrics ecosystem.

Second, Acute North diversified the services that could be delivered using the telegeriatrics platform. For elderly care, often the nursing homes conducted mortality audits (to discuss patient's dying conditions such as pain management or last wishes and areas of improvement) and multidisciplinary meetings (aimed to discuss patient's progress on areas such as discharge planning or ongoing social concerns). These meetings were conducted with a team that consisted of volunteer doctors, staff nurses, social or rehabilitation workers and specialists. Acute North offered these services to be conducted over the tele-geriatrics ecosystem. As noted by the nurse director at NH1

"My staff nurses will prepare 2-3 cases for discussion whether it is multidisciplinary or mortality audits. Usually we have very good discussions. We hope to improve our care in future, because when we discuss the case, we know where is the gap what we didn't do"'- Nurse manager, NH1

Acute North also introduced family tele-consultations whereby families of elderly patients could also speak to the specialists on the progress of their wards.

"It can be breaking bad news and updating family...when a patient died while consultation... the family was very happy. At least someone was there. It shows one of the possibilities. - Senior consultant, Acute North

Further, Acute North introduced ad-hoc consultations. Since, so far the sessions for tele-consultations were scheduled before hand, nursing homes could now request a session in case of emergencies. For this, the operations team at Acute North would arrange for the next available doctor. This helped nursing homes to access specialist service on demand and also reduced the hassle of transferring the patients to hospitals. However, ad-hoc consultations were being effectively used in NH1 only. As noted by the nurse manager, NH1 
"These are ad hoc cases, unplanned ones...we have done quite a number of ad hoc.

We do this. This is the whole purpose of having this tele-geriatric."- Nurse manager, NH1

While mortality audits, multidisciplinary meetings and tele-conferences were additional services delivered by the ecosystem, Acute North also introduced Tele CNE (continuing nurse education) as a means to build collective nursing homes learning capability. In such sessions, experts were invited to present lectures to the nurses through the tele-conference system and nurses could question and engage with other partner homes. Nursing homes also took turns to present cases from their homes to help other nursing homes learn from their experiences. As noted by executive director, NH4

"We have to trust and trust and mutual engagement takes time. You have to create opportunities to keep people engaged and we see potential in this" Executive Director, NH4

\section{Tension: Existing vs New Commitments}

While the focus of Acute North in this phase was to sustain the ecosystem by expanding its members and services, the addition of new nursing homes, revived both the tensions of individual vs collective identifications and expected vs actual capabilities albeit less strongly as Northern SeniorCare had developed joint workgroup forums to align the identities and was also now more familiar with the general level of capabilities in most nursing homes. For example, it was observed that the new private nursing homes had different incentives than those of existing voluntary funded nursing homes. While the older homes were focused on providing care to their patients, private nursing homes were relatively more business minded. It would require additional effort to assimilate the new nursing homes with the collective goals and values of Northern SeniorCare. As noted by the Operations Executive,

"The older nursing homes ask how to care for their patients, the private ones ask what the procedure is, how to get consent. They actually treat it like a business... for 
the private nursing homes it is free and they are making money out of this, it is a business for them"- Operations Executive Acute North

Similarly, the inclusion of more nursing homes also raised the need to enhance some capabilities. For example, it was noted by one of the clinical educators that addition of new nursing homes required more sophisticated technology for conducting joint nurse trainings (Tele CNEs) as current systems could not support eight nursing homes together.

"We have problem with the system now as it sometimes cannot connect... Unlike before we could hold five nursing homes in one set... now we are working on splitting to two sessions of four plus four-Clinical Educator, Acute North

However, Acute North was working to resolve these issues as the base capabilities were already established. Instead, in this phase, Acute North was confronted with even more critical challenges arising from both environmental context and from nursing homes. With the evolving healthcare in the country, government had recently increased its focus on the quality of care in the elderly segment. As nursing homes had a huge density of aging population, government had introduced new regulations for nursing homes where they were required to meet mandatory new standards to improve their level of care. The government had also initiated a digital initiative to push nursing homes to store information digitally.

Since both nursing homes and Acute North were members of such overlapping healthcare ecosystems, this put immense pressure on nursing homes. Nursing homes, with their limited resources, were struggling to continue to support the existing tele-geriatrics ecosystem and also the new initiatives arising outside the tele-geriatrics ecosystem. This was resulting in diminishing commitment from some nursing homes.

Similarly, Acute North being the anchor hospital for the northern region, was also a part of other evolving ecosystems arising from new government initiatives. With the opening of a new community hospital that aimed to provide rehabilitation and sub-acute services, 
some of Acute North's resources, such as the senior operations manager, were shifted from the tele-geriatrics ecosystem to run the new community hospital. Such changes resulted in the more dominant tension of existing vs new commitments for the entire ecosystem.

\section{Adaptive Orchestration Actions}

Acute North hence engaged in the adaptive orchestration action of aligning with external initiatives to reduce the tension of existing vs new commitments.

Aligning with external initiatives: Acute North started to explore ways to align itself with other initiatives. After a recent audit from the the government the nursing homes had received a detailed report card which had highlighted the key areas of improvement such as dignity of care, psychosocial and mental health. One such area was Advance Care Planning to help patients make their own decisions for future healthcare. Together with nursing homes, Acute North chartered training on how to communicate with end of life patients. Acute North thereafter set up a special clinic for nurses' training and piloted it across different departments such as geriatrics, dementia and diabetes center.

As noted by senior consultant at Acute North,

"Ministry of Health has now given a mandate so that nursing homes meet advanced standards. They have identified seven in priority. We help them develop guidelines.

\section{We also need them to be involved."- Senior Consultant, Acute North}

In addition, Acute North also started to involve itself in IT enablement ecosystem for nursing homes. This ecosystem would allow nursing homes to become more integrated across different ecosystems by ensuring timely flow of electronic information. Nursing homes would also be able to automate the processes and documentation which would reduce the constraint on nursing homes resources. Acute North sensed the increased operational efficiency by developing a link to this ecosystem. Acute North hoped to transfer some of the tele-geriatrics manual documentation to automation. 
"Government interventions are major game changers. Maybe once the electronic records system) is ready, they will have a game changer. It is still expensive and doctors have to realize the purpose..."- Senior Operations Manager, Acute North

As nursing homes were being pulled into these new initiatives, they struggled to meet these commitments with their limited capabilities. For example, in order that the elderly patients receive consistent quality of care, the government had initiated enhanced nursing home standards. For nursing homes to achieve these standards it was important that nursing staff was appropriately trained. Sensing an opportunity to be involved, Acute North extended its clinical educators to support nursing homes in these trainings. Since the nursing homes had already built relationships with clinical educators, they readily came on board for training. Acute North then formed an additional training steering committee with representatives from all nursing homes to particularly discuss nursing homes needs as they went through different trainings. As noted by Nurse director at NH3

"All nursing homes sit down and discuss which areas we want to focus first and where we need help. It's exciting. We are meeting every 2 weeks to see how training can be done"- Nurse director, NH3

With such changes in the healthcare landscape, Acute North kept itself aligned and more supportive of partner commitments.

\section{DISCUSSION}

This study examines in detail an integrated care case to explicate how the keystone organization manages the evolution of an ecosystem while addressing the emerging tensions.

Our findings show that the ecosystems evolve over time through three broad phases of evolution- member enrollment, joint capabilities development and ecosystem renewal (see Figure 1). In the first phase, the keystone organization engages in routine orchestration actions of initiating the ecosystem and seeking potential partners. With partners on board, in 
the second phase, the keystone organization focuses on developing the necessary capabilities to make the ecosystem operational. For this, the keystone organization engages in developing technology and knowledge resources and then establishes the relevant shared processes. Thereafter, in the third phase the keystone organization sustains the ecosystem by increasing its resilience against internal and external changes. For this, the keystone organization monitors the ecosystem performance and expands the ecosystem by addition of new members or products and services.

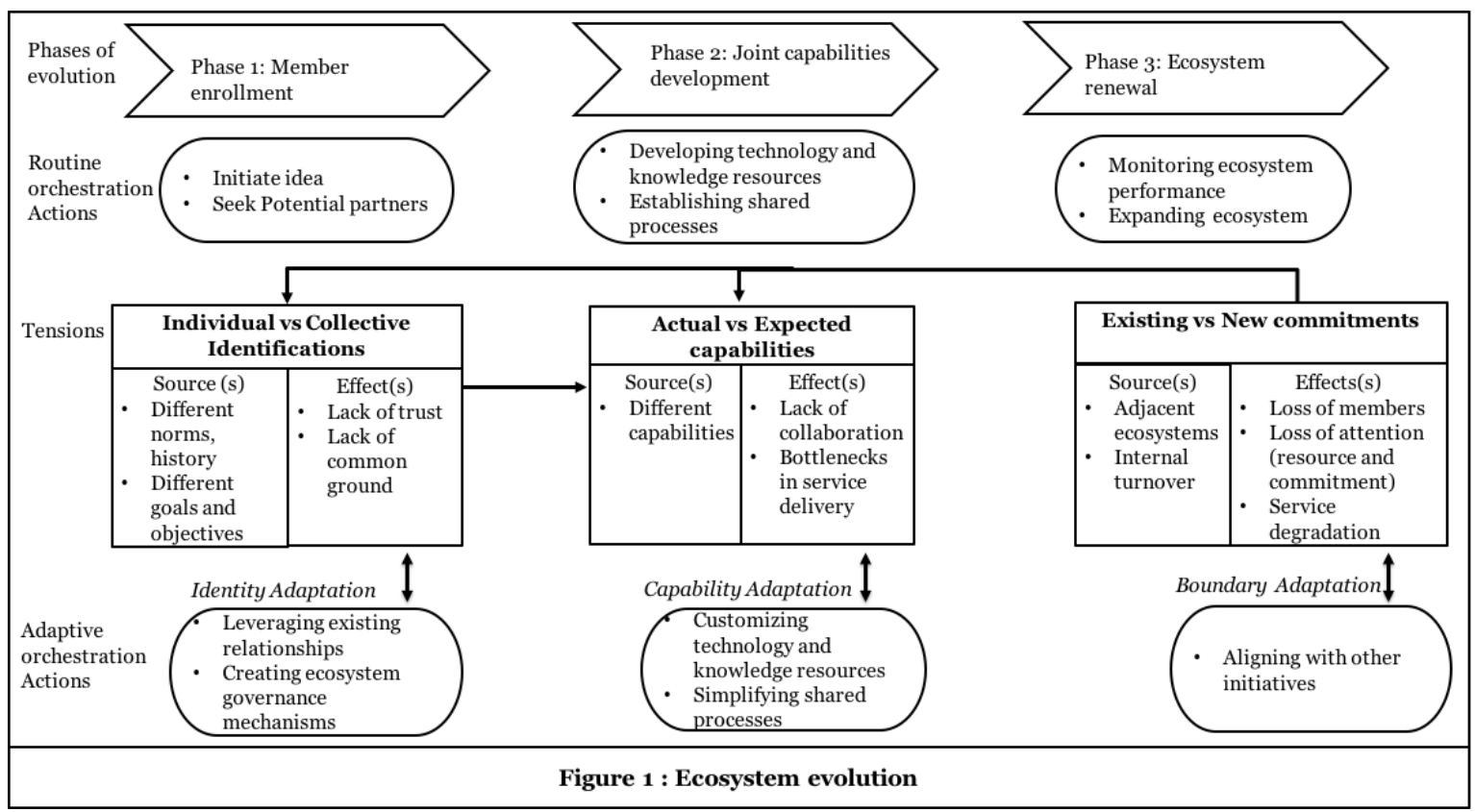

The three phases and routine orchestration actions observed are consistent with prior research (Jha et al. 2016; Leong et al. 2016; Srivastava and Shainesh 2015; Tan et al. 2015). However, our process model shows that as the keystone organization engages in these routine orchestration actions, things do not proceed smoothly. Challenges arising from external environmental context and internal diversity surface in the ecosystem. These challenges manifest as tensions or competing demands among members in the ecosystem. While a few studies have noted that tensions exist in ecosystem (Garud et al. 2002; Lindgren et al. 2015; Wareham et al. 2014), they have not specifically highlighted the dynamics of tensions during evolution, nor how tensions influence the evolution of the ecosystem. We found different 
tensions being more salient at different phases of evolution. Our findings reveal that as the keystone organization responds to these tensions, it engages in another set of adaptive orchestration actions involving ecosystem members, which in turn leads the ecosystem into the next phase of evolution.

We found three tensions: individual vs collective identifications, actual vs expected capabilities, and existing vs new commitments. While each tension was present throughout the ecosystem evolution, specific tensions were more salient in each phase. In the first phase of member enrollment, the members' disparate identities and lack of identification with the ecosystem had to be addressed in order for the ecosystem to get off the ground. In the second phase, the tension between actual and expected capabilities among members had to be addressed in order for joint capabilities to be effectively developed. In the third phase, as the ecosystem settled into an operating rhythm, the pull from new commitments arising from shifts in the external environment became salient, threatening the ongoing evolution of the ecosystem if left unaddressed.

The keystone organization responded to these tensions by engaging in corresponding adaptive orchestration actions in each phase of evolution. We labelled these actions Identity Adaptation, Capability Adaptation and Boundary Adaptation, reflecting the nature of the tensions that the actions sought to address. Conceptually, our findings suggest that adaptive orchestration actions differ from the routine orchestration actions both in their purpose and nature. While routine orchestration actions are standard actions focused on developing the ecosystem (Dhanaraj and Parkhe 2006), adaptive orchestration actions are dynamic and iterative focused on resolving the paradoxical tensions (competing demands). As these competing demands have to be simultaneously addressed, adaptive orchestration actions entail more creativity and flexibility on part of the keystone organization (Smith and Lewis 2011).These are also suggestive of stronger involvement of member feedback as the sources 
of tensions often lie with the partners. By resolving the tensions, the adaptive orchestration actions not only enable a smooth transition of the ecosystem from one phase to another, but also enrich the ecosystem by furthering opportunities as these enable learning (Smith and Lewis 2011). For example, the boundary adaptation actions in the third phase of evolution has moved the ecosystem beyond only provision of tele-geriatrics services, to being an ecosystem of equipping members with skills through its established training processes for meeting new regulatory and shifting industry norms. In the following sections, we now discuss in detail two critical findings of ecosystem tensions and adaptive orchestration actions.

\section{Ecosystem Tensions}

We now examine the three salient tensions in detail along with their source and effects. Prior literature on tensions (Smith and Lewis 2011) suggests that tensions are triggered from plurality (competing goals leading to uncertainty), change (availability of new options and competing opportunities) and scarcity (allocation decisions on scarce resources). Consistent with this, our findings reveal specific sources of tensions related to plurality, changes and scarcity in the internal, and external context of the ecosystem. At the same time, our findings also show the effect of these tensions which created bottlenecks in the evolution of the ecosystem.

In the first phase, the key tension is individual vs collective identifications. This tension occurs between the existing identities and incentives of individual organizations and their constant need to be aligned to a new collective ecosystem identity and incentive. This tension has been noted in prior studies where Wareham et al (2014) suggest that while organizations are driven by their individual incentives, they still need to jointly deliver services to the end customer. Lindgren et al (2015) particularly explicate the identity aspect of this tension which stems from differences in organization's history and mission. This in 
turn affects cooperation in the ecosystem. We elaborate on this by explicitly identifying sources and effects of this tension.

Consistent with prior research (Wareham et al. 2014) we found that this tension is first triggered due to plurality reflected in the diverse identities (history, mission and vision) and incentives of organizations. In this study, Acute North was a public sector, government subsidized hospital offering extensive range of medical services and healthcare. Nursing homes on the other hand were non-profit charitable organizations affiliated to different religious institutions providing long term care to the elderly people. There were also differences in the incentives these organizations sought from the ecosystem. While Acute North was more concerned on reducing unplanned admissions, nursing homes looked forward to benefit their residents by providing them access to specialist care. In addition, it was also observed that the tension was also triggered due to changes in external environmental context as rising healthcare needs and lack of skilled nursing resources pushed the nursing homes to rely on acute hospitals despite sharing different identities.

Our findings further show that, this tension resulted in both lack of trust and lack of common ground among the members. When Acute North approached nursing homes to join the ecosystem, nursing homes were highly skeptical of Acute North's intentions. For them, Acute North was a government-supported service provider with sophisticated resources, whereas they were charitable organizations with relatively low resources, providing long term care. They were apprehensive that Acute North might behave authoritatively and might also be insensitive to their historical vision of helping elderly people. Also, their main incentive to join the ecosystem was to benefit the free specialist consultations, Acute North however was fixated on reducing the burden on its resources. The relationships in this phase were hence very fragile as seen in the case of one nursing homes which dropped out citing lack of management support. 
In the second phase, the salient tension is that of actual vs expected capabilities.

This tension occurs due to incongruence between actual capabilities of members and the capabilities required to make the ecosystem operational. Since the members need to be complementary providers of services, their existing capabilities may be insufficient to meet ecosystem demands. Past research has extensively highlighted the role of capabilities in ecosystem development (Sarker et al. 2012; Tan et al. 2015). However, their focus has been on suggesting the critical capabilities required in an ecosystem from all members. There has been relatively little consideration of the dissonance resulting from differences in actual member's capability vs required ecosystem capabilities.

This tension becomes particularly salient in the second phase when the ecosystem needs to develop the necessary capabilities to become operational. While the members bring in the complementary resources and capabilities, often these have to be further developed in combination with the keystone organization's capabilities to meet ecosystem needs. For example, we found that Acute North was initially aware that nursing homes lacked clinical capabilities to diagnose and treat their patients, however when Acute North started developing materials for nurse training, the nursing homes found the training difficult to adopt. This clearly displayed the lack of understanding members had of each other's capabilities. At the same time, resource scarcity arising from the external context also rendered the tension salient. For example, in this study, we saw that the lack of geriatrics trained nurses widened the gap between actual and expected capabilities.

If the tension is not addressed, it effects the creation of ecosystem capabilities which in turn will not allow members to work together effectively. In this study, we found that it was imperative for Acute North to take a more customized approach in the creation of needed capabilities by developing a more simplified set of knowledge and technology resources in order to jointly deliver tele-geriatrics services. 
In the third phase, the key tension is existing vs new commitments. Members in an evolving industry may simultaneously be involved with multiple ecosystems (Eisenmann et al. 2006). For example, our study showed that in addition to the tele-geriatrics ecosystem, the nursing homes were also a part of an evolving healthcare industry comprising of other nursing homes and the Ministry of Health. The recent changes in the environmental context where the government introduced multiple initiatives to raise nursing homes standards resulted in competing opportunities and threats for nursing homes. An existing ecosystem faces an envelopment threat when adjacent ecosystems expand and attract its members. Although ecosystem threat is more common in the context of competing platforms, ecosystems may still find it difficult to retain member commitment with their increased participation in other ecosystems.

This also raises the additional concern of scarcity as members have scarce resources but multiple ecosystem commitments. For example, in this study, when the Health Ministry initiated an ecosystem of enhanced nursing standards, nursing homes faced the dilemma of how to commit scarce resources to multiple ecosystems. If the tension is not addressed, it may result in loss of members or degradation in existing services. In fact, the number of teleconsultations took a dip in two nursing homes as their participation in adjacent ecosystems increased. Together, both changes in external environmental context and resource scarcity were crucial sources of tension in this phase.

In addition to the key tensions that were salient in each phase, our findings also suggest the presence of other tensions although in a slightly lower magnitude. It was observed that in the second phase while the key tension was actual vs expected capabilities, the tension of individual vs collective identifications also impaired the process of capability development. For example, when Acute North started developing joint capabilities with the nursing homes, the differences in cultures and history of members created bottlenecks. While 
nursing homes did not have standard ways of collecting data or managing patients, Acute North reflected a more process oriented way of delivering services. Hence a new set of common procedures for the ecosystem had to be developed from scratch.

Similarly, in the third phase when the ecosystem sought expansion with inclusion of new members and services, it revived both the tensions of actual vs expected capabilities and individual vs collective identifications. For example, the new private nursing homes were found to have different individual goals and their alignment to collective identity of the ecosystem was necessary. Also, with eight nursing homes, the technology capabilities had to be enhanced to support all the homes for online trainings at the same time. However, these tensions were of slightly less intensity now as Northern SeniorCare had developed joint workgroup forums to align the identities and was also now more familiar with the general level of capabilities in most nursing homes (See arrows connecting the tensions in Figure 1).

While the prior research (Banoun et al. 2016; Garud et al. 2002; Lindgren et al. 2015; Wareham et al. 2014) has identified tensions of individual vs collective identifications, standard vs variety and autonomy vs control, our findings affirmed the first tension of individual vs collective identification, and revealed two other tensions of actual vs expected capabilities and existing vs new commitments. We believe that the additional tensions observed were due to the fact that this was a service-based ecosystem (Lusch and Nambisan 2015). The tensions of standard vs variety, and autonomy vs control, identified in the prior research were more salient in the context of goods-based ecosystems (Wareham et al. 2014) where the focus is on separation and control of organizations (Lusch and Nambisan 2015). In goods-based ecosystem, standards vs variety tension arose from the need to balance the standard core platform with complement components developed by the members. This also raised the tension of autonomy vs control where the autonomy given to organizations to create innovations had to be balanced with the control over quality of innovation. 
Service ecosystem on the other hand, requires organizations to exchange their respective capabilities to jointly deliver services. For example, in this study, nursing homes' initial assessment of patients is exchanged with more sophisticated diagnosis of Acute North. Together this exchange provides joint consultation to the patients. Even innovation in the form of ad-hoc consultations requires members to jointly exchange capabilities. This exchange hence raises the tension of actual vs expected capabilities in the service ecosystem as lack of expected capabilities results in inability to jointly deliver services.

This also necessitates member's commitment to the service exchange as dilution of both resources and capabilities may result in low quality of exchange and eventually loss of joint service delivery. For example, in this case, we saw that as nursing homes started committing resources to newer initiatives, it resulted in diminishing tele-geriatrics services to the patients. Again, this becomes a salient issue in service ecosystems, as the output of collaboration is determined by the commitment of members.

While these two tensions became salient in this study, we believe tensions of standard vs variety and autonomy vs control could also arise in service ecosystems. For example, members may contest over the need to standardize resources or processes and may also seek control over conducting shared processes, and have autonomy over their own processes. However, in this study, these tensions did not become salient. We believe this was partly because Acute North had proactively orchestrated the ecosystem by giving voice to members in shared processes as well as by giving them autonomy over certain processes such as selection of patients for tele-consultation.

In summary, our findings suggest that salient tensions exist in each phase of ecosystem evolution and tension in one phase may revive the tension in another phase of evolution. Second, while prior literature recognizes three tensions inherent in ecosystems, our findings reveal that as service becomes essential to ecosystems, there are additional tensions 
that are critical to ecosystems- actual vs expected capabilities and existing vs new commitments. These tensions arise when members exchange service which requires both member capabilities and commitment.

\section{Adaptive Orchestration Actions}

Our review of the literature led us to identify a set of orchestration actions taken by the keystone organization. We label these actions as routine orchestration actions as these are necessary to steer the ecosystem during each phase of evolution. In this study, we extend this to highlight the additional adaptive orchestration actions taken by the keystone organization in response to the tensions in each phase of evolution.

These orchestration actions are adaptive as these are particularly enacted to respond to tensions and are emergent from interaction among the members and the keystone organization. As the keystone organization responds to the tensions, it shapes the evolution of the ecosystem. For example, if the tension of individual vs collective identifications is not balanced in the first phase, the keystone organization would not be able to evolve to the second phase of joint capabilities development due to lack of trust arising from the tension. Adaptive orchestration actions subsume the effect of tensions and steer the ecosystem to the next phase of evolution.

In the first phase of member enrollment, the literature suggests that the keystone organization first assesses the demand, develops a value proposition and then seeks potential partners for the ecosystem (Jha et al. 2016; Srivastava and Shainesh 2015; Tan et al. 2015). While these actions are necessary, they are not sufficient to address the tension of individualcollective identifications. This is because routine orchestration actions are purposed to evolve ecosystem towards its shared purpose. In this case we saw that the keystone organization assessed demands of care from the nursing homes, developed a tele-geriatrics proposition and then invited partnerships from nursing homes. However, the keystone organization also 
engaged in another set of adaptive orchestration actions that we label Identity Adaptation.

These actions were specifically taken to manage the tension of Individual vs Collective identifications. This included leveraging existing relationships to reduce the mistrust between members and the keystone organization and then creating ecosystem governance mechanisms such as decision making forums to steer strategy and maintain transparency in the ecosystem. Together, these actions aligned the members towards a collective identity and aligned their incentives. Table 3 summarizes the tension and the adaptive orchestration actions

Table 3: Member Enrollment: Tension and Identity Adaptation

\begin{tabular}{|c|c|}
\hline Tension & Adaptive Orchestration (Identity Adaptation) \\
\hline \multirow[t]{2}{*}{$\begin{array}{l}\text { Individual vs Collective } \\
\text { identifications } \\
\text { (Acute North was highly } \\
\text { resourced provider of } \\
\text { acute short-term care } \\
\text { and nursing homes were } \\
\text { step-down facilities } \\
\text { providing affordable } \\
\text { long-term care) }\end{array}$} & $\begin{array}{l}\text { Leveraging existing relationships } \\
\text { - Enrolling partners with prior relationship } \\
\text { - } \text { Identified existing contracts with nursing homes } \\
\text { - } \text { Bring in trustful resources (operations manager with } \\
\text { prior experience in nursing homes) to pitch talks } \\
\text { - Prioritizing partners (more likely to agree and } \\
\text { succeed) } \\
\text { - Approach NH1 } \\
\text { - Offer Tele- geriatrics as a free add on service } \\
\text { - NH1 evaluates benefits of proposal \& accept } \\
\text { Using early member influence to bring new } \\
\text { members } \\
\text { - NH2 approached Acute North for tele-geriatrics } \\
\text { - NH3 and NH4 approached by Acute North (existing } \\
\text { service contract \& accept the proposal } \\
\text { - } 2 \text { more NH approached ( no service contract but } \\
\text { dropped out) }\end{array}$ \\
\hline & 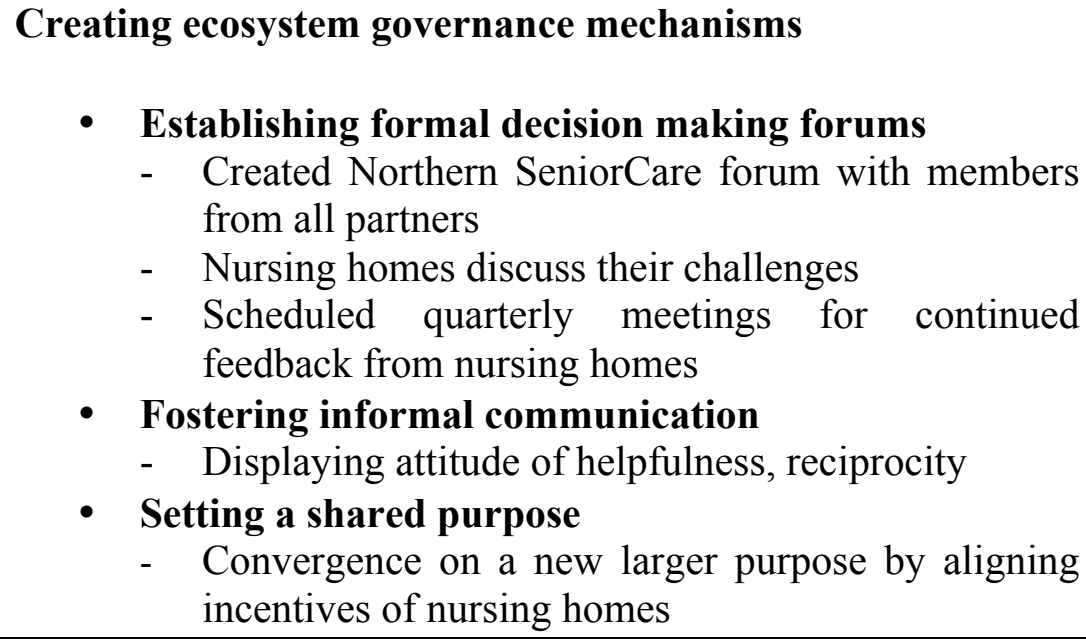 \\
\hline
\end{tabular}


In the second phase of joint capabilities development, the literature recommends that the keystone organization engages in the development of technology platform, resources and shared processes (Cusumano and Gawer 2002; Leong et al. 2016; Srivastava and Shainesh 2015; Tan et al. 2015). The keystone organization develops the knowledge routines and also develops common technology platforms to increase modularity and interoperability among the members. Our case similarly showed that Acute North engaged in providing nursing homes necessary training and piloting solutions to develop the infrastructure for conducting tele-consultations. However, vanilla implementation of standard resources and processes was difficult in this case due to members' limited capabilities. We saw, for example how nursing homes found the standard training materials to be too medically oriented and way above their level of comprehension. Acute North hence engaged in more adaptive set of orchestration actions which we term as Capability Adaptation. These actions were particularly directed to reduce the challenges members faced in leveling-up their capabilities. For example, to train nursing homes, Acute North hired clinical educators who revised the training content to make it more nurse oriented. Thereafter, the training sessions were split into two sessions to make it easier for nursing homes. Further, more handholding was done as English was not the first language for many nurses. Therefore, by enacting two specific actions of customizing technology and knowledge resources and simplifying shared processes, the keystone organization was able to balance the tensions. Table 4 provides a summary of tension and adaptive orchestration actions.

Table 4: Joint Capabilities Development: Tension and Capability Adaptation

\begin{tabular}{|l|c|}
\hline Tension & \begin{tabular}{c} 
Adaptive Orchestration (Capability Adaptation) \\
\hline $\begin{array}{l}\text { Actual vs Expected } \\
\text { capabilities }\end{array}$
\end{tabular} \\
$\begin{array}{r}\text { Customizing technology and knowledge resources } \\
-\quad \text { Developing customized technology resources } \\
-\quad \text { Gathered user requirements from nursing homes }\end{array}$ \\
\hline
\end{tabular}




\begin{tabular}{|c|c|}
\hline $\begin{array}{l}\text { (Acute North's } \\
\text { expectation of nursing } \\
\text { homes capabilities was in } \\
\text { contrast to nursing } \\
\text { homes' ability to deliver) }\end{array}$ & $\begin{array}{c}\text { - } \text { Nursing homes fed back their technology limitations } \\
\text { - } \text { Installed cable broadband for internet } \\
\text { - } \text { Pilot tested low cost technology for consultation } \\
\text { - } \text { Nursing homes requested simplified version (TV set) } \\
\text { - } \text { Procured tele-conference equipment for consultation } \\
\text { - } \text { Nursing homes raised design issues in mobility } \\
\text { - } \text { Introduced trolley design for bedside patients } \\
\text { - Developing customized knowledge resources } \\
\text { - } \text { Nursing homes requested less medical training course } \\
\text { - } \quad \text { Revised course to increase practical component, and } \\
\text { - } \text { simplify theoretical aspects } \\
\text { Simplifying shared processes } \\
\text { Tele-consultation process } \\
\text { - Operations team at Acute North established point of } \\
\text { - } \text { contact with nursing homes } \\
\text { - } \text { operations team } \\
\text { Shared responsibilities with nursing homes for the } \\
\text { Repocesses } \\
\text { - Nursing homes collected KPIs in flexible formats } \\
\text { - Operations team at Acute North formatted and } \\
\text { submitted reporting numbers to government }\end{array}$ \\
\hline
\end{tabular}

In the third phase of ecosystem renewal, prior literature suggests that the keystone organization engages in monitoring the ecosystem performance and diversification of ecosystem services (Dhanaraj and Parkhe 2006; Gausdal and Nilsen 2011; Leong et al. 2016; Moore 1993) to make the ecosystem more valuable for the members. In this case, we saw that Acute North indeed expanded its portfolio to offer nursing homes services such as mortality audits, multidisciplinary meetings over tele-geriatrics ecosystem. Acute North also monitored the ecosystem performance and found that two nursing homes had reduced their number of tele-consultations. However, while these actions were necessary, these were not sufficient to manage the emerging tension of existing vs new commitments. Hence we found that the keystone organization engaged in more adaptive orchestration actions which we term as Boundary Adaptation. The rise of related ecosystems affected nursing homes commitment to 
tele-geriatrics ecosystem more so because of their limited resource base. Hence Acute North started to explore ways to align its ecosystem to these newer initiatives. For example, Acute North looked forward to connect to the complementary digital information ecosystem in order to make tele-geriatrics processes more efficient. In addition, Acute North also leveraged its existing capabilities to ease the alignment. Acute North took cognizance of member challenges and discussed these in governance forums. Acute North then offered the services of its clinical educators for other training programs of nursing homes. Table 5 summarizes the tension and adaptive orchestration actions.

Table 5: Ecosystem Renewal: Tension and Boundary Adaptation

\begin{tabular}{|c|c|}
\hline Tensions & Adaptive Orchestration (Boundary Adaptation) \\
\hline $\begin{array}{l}\text { Existing vs new } \\
\text { commitments } \\
\text { (Nursing homes } \\
\text { commitment was } \\
\text { required for both } \\
\text { existing tele- } \\
\text { geriatrics } \\
\text { ecosystem and } \\
\text { new external } \\
\text { initiatives) }\end{array}$ & 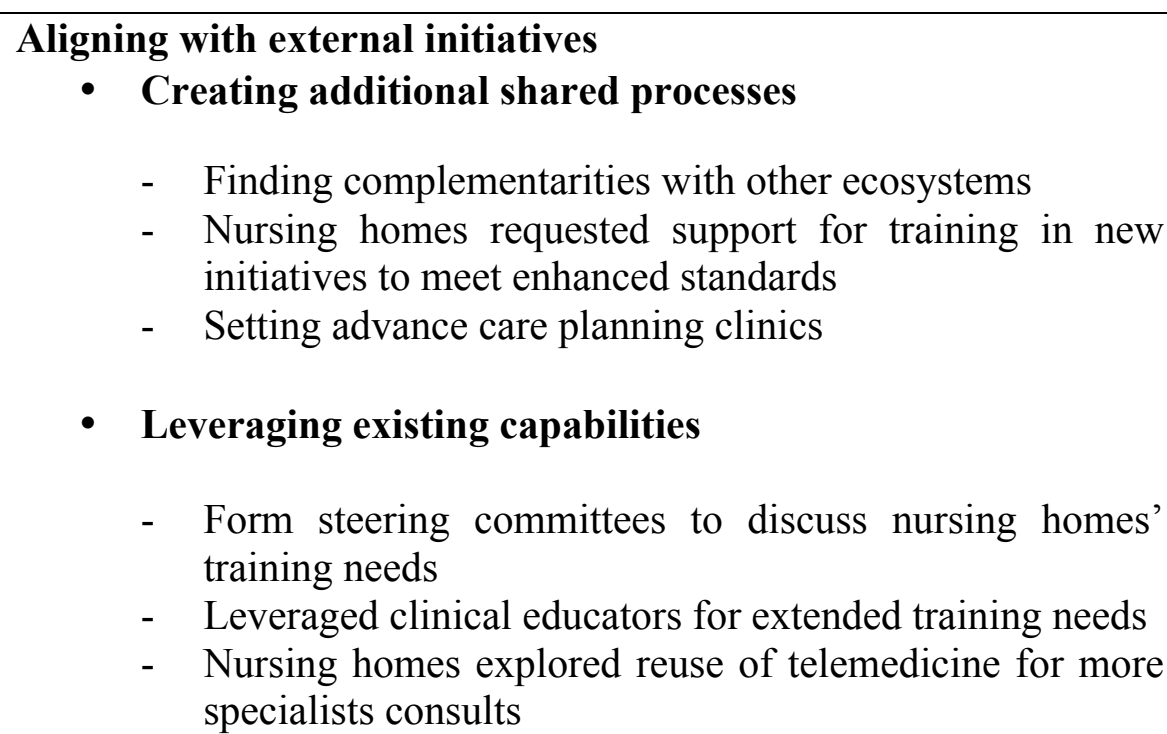 \\
\hline
\end{tabular}

In summary, adaptive orchestration actions are enacted in response to the tensions and are suggestive of much stronger involvement of members through feedback and interactions. These are adaptive as they incorporate feedback loops which result in emergent resources and processes. This adaptation was also noted by Garud et al, 2002 in their study on Sun Microsystems where Java emerged as a standard through actions taken by the keystone 
organization in response to member concerns. For example, to avoid fragmentation, members using Java to develop applications had to undertake several compatibility tests. However, this resulted in trust issues among the members and many deflected from the standard as they suspected excessive control of Sun Microsystems. Sun Microsystems hence reorganized its business and separated standardization effort from application development. It also modified its licensing model to provide flexibility to members. This adaptation to member feedback was critical in managing the tensions. These adaptive orchestration actions are different from routine actions which are initiated and driven by the keystone organization alone to facilitate the development of ecosystem. While the literature recognizes these routine actions (Batterink et al. 2010; Dhanaraj and Parkhe 2006; Nambisan and Sawhney 2011; Prince et al. 2014), we additionally provide a toolkit of adaptive orchestration actions which are emergent in response to tensions and complement the routine actions. Together both routine and adaptive orchestration actions guide the keystone organization on how to orchestrate a sustainable development of ecosystem.

\section{Theoretical Contributions}

This study contributes to literature in ecosystems evolution in the following ways. First, prior research has suggested that ecosystems evolve (Jha et al. 2016; Leong et al. 2016; Srivastava and Shainesh 2015; Tan et al. 2015), often through the actions of the keystone organization (Dhanaraj and Parkhe 2006; Iansiti and Levien 2004). It has also been highlighted that this evolution is subjected to challenges or tensions arising from internal and external environment (Garud et al. 2002; Lindgren et al. 2015; Wareham et al. 2014). However, these two aspects have been dealt with separately. Our study reconciles these together to provide a longitudinal view of tensions during evolution. In doing so, it identifies three salient paradoxical tensions, namely, individual vs collective identifications (member enrollment phase), actual vs expected capabilities (joint capabilities development phase), and 
new vs existing commitments (ecosystem renewal phase), their sources and effects on the ecosystem. Our process model of ecosystem evolution provides a more compact view of ecosystem evolution.

This study extends the concept of tensions identified in the broader organization literature in a single organization to more complex level of ecosystems. Consistent with prior research, we observe that the triggers of plurality, change and scarcity (Smith and Lewis 2011) arise not only from external environment, but also from inherent ecosystem characteristics of diversity and interdependence. In doing so, we identity two specific tensions of actual vs expected capabilities and existing vs new commitments which are more relevant in the context of ecosystems.

The study also adds to the recent stream of research that has started to examine the tensions that impact the ecosystem evolution (Garud et al. 2002; Lindgren et al. 2015; Wareham et al. 2014). In addition to the tension highlighted in the previous literature such as individual vs collective identifications (Wareham et al. 2014), this study suggests specifically two more tensions which become prevalent especially in the case of service ecosystems (Lusch and Nambisan 2015). First is the actual vs expected capabilities which occurs due to incongruence between actual capabilities of members and the capabilities required to make the ecosystem operational. While the previous literature does suggest that members bring in complementary resources to the ecosystem (Sarker et al. 2012) which are then used jointly to deliver services, less is known on the issues arising during this integration. Our study suggests that member's expectations of each other capabilities and the actual on ground capabilities might result in tensions which could be resolved though levelling up of capabilities. Second is the existing vs new commitment tension which occurs due to member's commitment to multiple ecosystems. While this has been suggested as a challenge to sustainability in the context of platform competition (Eisenmann et al. 2006), our findings 
suggest that this challenge may exist whenever members are part of multiple ecosystems. The tension becomes even more salient in service ecosystems, where member commitment to service exchange is critical to quality of service being delivered.

Last, very importantly, the study also adds to and extends the literature on ecosystem orchestration (Batterink et al. 2010; Dhanaraj and Parkhe 2006; Nambisan and Sawhney 2011; Prince et al. 2014) which has mostly focused on the routine orchestration actions that are initiated by the keystone organization alone to evolve the ecosystem. While most studies have suggested a varying list of routine orchestration actions, our findings add to the recent stream of work in orchestration (Batterink et al. 2010; Dhanaraj and Parkhe 2006; Nambisan and Sawhney 2011; Prince et al. 2014) that has started to propose some broad sequences in the activities that an orchestrator performs. Our study specifically highlights this sequence of routine orchestration actions in each phase of evolution.

Second, our research reveals that along with necessary routine orchestration actions, the keystone organization enacts another set of adaptive orchestration actions to address the tensions that arise in each phase of evolution. These adaptive orchestration actions are in response to the tensions and are adaptive in nature as these incorporate member concerns and feedback over time. By highlighting both the routine and adaptive orchestration actions together, our study provides both a clear distinction between two types of orchestration actions and also an explanation on how these interact during evolution.

\section{Practical Implications}

Practically, our findings have several implications for the keystone organization. First, it sensitizes the keystone organization to the notion of specific tensions that are salient in each phase of ecosystem evolution, and how these could be addressed with adaptive orchestration actions. Our findings reveal that these tensions are likely to be observed in contexts where members differ significantly in their identities, goals and capabilities and are 
part of multiple ecosystems. Specifically, we found that the keystone organization needs to be emphatic and observant of member needs lest they damage the ecosystem stability. A possible approach taken by the keystone organization could be to facilitate communication with members to discuss challenges faced by them. Also, the keystone organization needs to vigilant to rising ecosystems in the industry and continue to maintain a valuable proposition (through diversification of services if needed) for its members.

Our findings also have implications for integrated care. With diversity in providers and scarcity of resources, integrated care is fraught with continuous challenges of differences in organizational identities and incentives and lack of trusting relationships (Bevan and Janus 2011; Cebul et al. 2008; Leichsenring 2004; Ling et al. 2012a; Montenegro et al. 2011; Pate et al. 2010; Pike and Mongan 2014; Pomerantz et al. 2009). By considering implementation of adaptive orchestration actions such as developing governance mechanisms to align incentives and leveraging existing relationships to develop trust and collective identity, the keystone organization can address some of the challenges. Also, while this study is focused on telemedicine enabled integrated care, our process approach to ecosystem evolution, related tensions and findings may also be applicable to other types of integrated care settings which face challenges of resources and commitments.

\section{Conclusion and Future Research}

This research is motivated to ascertain how the keystone organization manages the evolution of an ecosystem by addressing the emergent tensions. We draw on an integrated care case to surface the salient tensions and related orchestration actions in each phase of ecosystem evolution. The study has strong implications on the role of the keystone organization in enacting a set of adaptive orchestration actions along with the necessary routine orchestration actions to manage the tensions. Our findings reveal a process model of evolution as a framework to identify and manage tensions. 
Future research can extend our findings and also address limitations of this study. While we explore this in the context of healthcare which is characterized by a resource scarce environments and public private partnerships, future studies must consider this to strengthen the generalizability of our process model. For example, in case of platform driven ecosystems which have thousands of partners, the keystone organization might enact additional set of adaptive orchestration actions. Further studies could build on the repertoire of adaptive orchestration actions that we have identified. 


\section{CHAPTER 3.}

\section{VALUE CO-CREATION IN SERVICE ECOSYSTEMS: A MEMBER'S \\ PERSPECTIVE}

\section{INTRODUCTION}

There has been a considerable shift across the globe from a producer centric and goods-based economy to a more consumer-centric and service based economy (Barrett et al. 2015; Lusch and Nambisan 2015; Rai and Sambamurthy 2006; Vargo and Lusch 2004). Given this paradigm, service has become fundamental to economic exchange among organizations (Lusch and Nambisan 2015; Vargo and Lusch 2004). Service here refers to the specialized competencies that each organization brings in the ecosystem. These are then exchanged to provide mutual benefits. For example, an organization may exchange its ability to market a product with another organization that can provide logistics for delivery in rural areas. These service exchanges enable organizations to co-create value together by forming a service ecosystem and accessing critical resources and capabilities that lie outside their boundaries (Adner 2006; Lusch and Nambisan 2015).

A service ecosystem is defined as "a relatively self-contained, self adjusting system of loosely coupled social and economic actors connected by shared institutional logic and mutual value creation through service exchange" (Lusch et al $2015 \mathrm{p} \mathrm{161).} \mathrm{Members} \mathrm{in} \mathrm{a}$ service ecosystem co-create value by engaging in service exchanges over time. This is often facilitated by a keystone organization that together with the members develops necessary processes and resources. Service ecosystems are also characterized by service platforms which enable the members to interact and coordinate their exchanges (Adner 2006; Lusch and Nambisan 2015). For example, organizations such as IBM have launched several 
partnership programs which allow members to join their ecosystems, access their technology platform and further build solutions by sharing their competencies (Ceccagnoli et al. 2012).

Organizations are increasingly participating in such ecosystems to improve their business performance (Adner 2006). While participation in ecosystems brings value to the keystone organization by increasing its portfolio and reach of services (Jha et al. 2016; Leong et al. 2016; Sarker et al. 2012; Tan et al. 2015), recent research has started to specifically explore the value to members from participation in an ecosystem (Ceccagnoli et al. 2012; Han et al. 2012). For example, member participation in an innovation ecosystem was found to improve member's market valuation (Ceccagnoli et al. 2012; Han et al. 2012).

There are however still important aspects of member value from ecosystem participation that remain relatively unexamined. First, only limited aspects of value have been studied. Value to members is mostly measured as changes in the market value of members when they join ecosystems (Ceccagnoli et al. 2012; Han et al. 2012). However, value in the form of member's financial performance (revenue or profits) or non-economic value (quality of service) still remains under-studied (Han et al. 2012; Sherer 2014). Second, while value co-creation is a process that unfolds over time, past empirical studies have largely been conducted with cross-sectional data viewing participation as a one-time event (Ceccagnoli et al. 2012; Han et al. 2012). Third, the influence of coordination of the exchange of resources and services and the development of symbiotic relationships among the members and the keystone organization has not been empirically examined. While studies have clearly indicated these exchanges as critical components to value co-creation (Grover and Kohli 2012; Sarker et al. 2012), yet how these result in value to members remains unexplored (Grover and Kohli 2012).

Understanding what influences member's value from participation in ecosystems is important for several reasons. First, for the ecosystem as a whole to thrive, it needs to attract 
and retain an adequate number of members. A key motivation for organizations to join ecosystems is to create value that they cannot develop on their own (Adner 2006). However, if members are unable to benefit from the partnership over time, they may leave and join other ecosystems. Second, it is also important for the members to understand how their participation with the keystone organization in the ecosystem can enable them to derive value from the ecosystem. Merely joining an ecosystem may not always result in value for them if they do not engage in ongoing value co-creation exchanges.

Hence in this study, we examine the question of how member participation in exchanges within the ecosystem influences the value to members. We do so in the context of integrated care (IC) in the healthcare sector, where diverse providers form a service ecosystem to provide continuity of care for patients. We analyze four-year panel data collected on the IC ecosystem, which was formed to deliver tele-consultations to geriatrics patients. This ecosystem comprised of an acute hospital (the keystone organization) and four nursing homes (participating members). We go beyond economic measures of value (e.g. cost effectiveness), to include other aspects of value appropriate to the IC context (e.g. clinical outcomes, user satisfaction).

Theoretically, we conceptualized the IC ecosystem as a service ecosystem where value is co-created through collaborative exchanges of services (or application of competencies) among diverse healthcare providers. For example, a primary care physician may exchange its preliminary diagnostics with a secondary care hospital's in-depth examination to provide holistic care to the patient. Taking this service view and broader value co-creation literature, we argue that member participation in three value co-creational exchanges (service, resource and relational) influences the value they receive. Service exchanges occur when member and the keystone organization exchange their respective application of competencies through joint processes (Lusch and Nambisan 2015; Vargo and 
Lusch 2008). Resource exchanges occur when members and the keystone organization exchange complementary (dissimilar) or supplementary resources (similar) such as knowledge and human capital (Sarker et al. 2012). Relational exchanges are communications and interactions (both formal and informal) among the members and the keystone organization which allow for development of a shared purpose, sharing of opportunities and resolution of disputes (Lusch and Nambisan 2015; Sarker et al. 2012).

The acute hospital in this case developed a tele-geriatrics platform which allowed nursing homes to receive specialist consultations for their geriatrics patients. We observe resource, relational and service exchanges in this service ecosystem, and then measure its effect on the value members derive from participation (clinical outcomes, user satisfaction and cost effectiveness).

In the following sections, we first provide an overview of integrated care ecosystem. We then take a service view of ecosystems to develop our hypotheses. This is followed by methods, results and discussion.

\section{THE INTEGRATED CARE ECOSYSTEM}

Many countries are experiencing rapidly aging populations and concomitant rise in multiple chronic illnesses (Curry et al. 2013). Diverse providers catering to different patient needs have arisen over time, resulting in fragmentation in delivery of healthcare services (Kodner and Spreeuwenberg 2002; Stange 2009; Stille et al. 2005). For example, primary care is often provided by general practitioners, secondary care is led by specialists, and tertiary care by hospitals providing highly intensive services usually for inpatients. In addition, social care services, rehabilitation and counselling service centres also serve patient's other related needs. This has led to increased healthcare costs as well as gaps in the delivery of care when patients need to receive care from multiple providers (Stange 2009). 
Integrated care has frequently been recommended as a solution to provide consistency and continuity in care by creating collaboration among different providers (Kodner and Spreeuwenberg 2002; Stange 2009; Stille et al. 2005). We conceptualize such integrated care collaborations as service ecosystems. First, similar to service ecosystems, integrated care collaborations comprise of loosely coupled actors (free to exit and enter into collaborations) who mutually co-create value by exchanging their services (Norman 2011; Pike and Mongan 2014). Services here refer to specialized competencies that each actor brings to the ecosystem such as specialist diagnostics or nursing care for chronically ill patients. Second, service ecosystem members develop a shared worldview and shared goals over time (Lusch and Nambisan 2015). In the integrated care context, these are usually continuity in care and/or efficiency in service delivery. To achieve this, the keystone organization often develops architectures for participation which facilitates coordination and alignment of incentives (Lusch and Nambisan 2015). Third, similar to service ecosystems, actors in integrated care usually integrate their resources to create more beneficial resources (Sarker et al. 2012; Srivastava and Shainesh 2015). For example, critical resources such as nurses and doctors may be integrated to deliver more forms of personal care. Fourth, these IC collaborations, like service ecosystems, are also self-adjusting which implies that actors continue to sense and respond to changes for continued sustainability (Minkman 2012; Srivastava and Shainesh 2015). Last, service platforms are often used to facilitate exchanges and interactions among all actors in a service ecosystem (Lusch and Nambisan 2015).

Integrated care ecosystems often rely on technology platforms such as telemedicine and electronic health records. For example, an integrated care ecosystem may utilize a telemedicine platform to deliver specialist services to rural areas (Srivastava and Shainesh 2015) or implement an electronic health records system to provide continuity in care to patients as they move from one provider to another (Pike and Mongan 2014). Telemedicine is 
one of the most widely used integrated care models, for providing specialist services such as geriatrics, cardiology to remote and/or community-based patients (Finkelstein et al. 2006; Roine et al. 2001). Telemedicine "involves the exchange of medical information from one site to another via electronic communications to improve a patient's clinical health status" (Yeow and Goh 2015). In other words, telemedicine allows for a joint medical assessment where the patient's medical information is shared between the nurses (usually with the patient) and doctors (distant specialists) over technology.

With the growth of telemedicine enabled ecosystems, research has started to assess the value from use by designing different methodologies such as randomized controlled designs(Finkelstein et al. 2006), surveys (Balasubramanian and Spurgeon 2012; Chae et al. 2001; Shahpori et al. 2011), experimental designs (de Stampa et al. 2014) cross sectional studies and meta-analysis (Nordheim et al. 2014; Roine et al. 2001). For example, Finkelstein et al, (2006) establish a randomized control design to monitor clinical outcomes and costs for a home healthcare system over a period of six months. Their results suggested that use of telemedicine improved clinical outcomes at lower costs than face-to-face visits. Similarly, in another study Chae et al (2001) conducted a survey on fifty elderly patients who were provided care through telemedicine. Their findings suggested that after telemedicine was introduced there was reduction in number of clinical visits as well as improved patient satisfaction.

Our review of various studies of telemedicine IC ecosystems suggests that members derive value in three forms - improved clinical outcomes (improvement in quality of life), user satisfaction and increased cost effectiveness (Balasubramanian and Spurgeon 2012; Busse and Stahl 2014; Chae et al. 2001; Goran 2012; Greaves et al. 2013; Jacobs et al. 2012; Larcher et al. 2003; Mayhew 2009; Sandoval Garrido et al. 2014). While there has been significant research that has evaluated the changes in these value indicators after telemedicine 
has been implemented (Balasubramanian and Spurgeon 2012; Becevic et al. 2015; Rojas and Gagnon 2008; Whittaker and Wade 2014), relatively few studies have considered the impact of complex exchanges among the members of the ecosystem, which might influence the value to members in such healthcare settings (Ignatowicz et al. 2014; Ruesch et al. 2012; Sherer 2014). With this understanding of the IC context, we now present the service dominant view of value co-creation to explicate value co-creational exchanges in a service ecosystem, and then develop a set of hypotheses on the relationship between such exchanges and value to members.

\section{SERVICE ECOSYSTEM AND VALUE CO-CREATION}

Value co-creation is a fundamental reason for economic exchanges among organizations. A service dominant view of value co-creation suggests that organizations cocreate value by forming a loosely coupled (members are free to enter and exit) service ecosystem where they engage in exchange of service (Lusch and Nambisan 2015; Vargo and Lusch 2004; Vargo and Lusch 2008). Service exchange here refers to the exchange of application of competencies by organizations. For example, an organization's ability to manufacture a product may be exchanged with another's competency to sell it in the rural markets. Together, by exchanging the application of their respective competencies, organizations mutually co-create value for the end customer. However, this service exchange also necessitates that organizations share symbiotic relationships with each other by developing shared goals, exchange resources such as knowledge and adjust to changes in internal and external environment together (Lusch and Nambisan 2015).

A service dominant logic to value co-creation hence is focused on exchanges between organizations and subsequent unfolding of value (Merz et al. 2013). Taking this service perspective and broader value co-creation literature, we explicate three exchanges - service exchange, resource exchange and relational exchange (Grover and Kohli 2012; Lusch and 
Nambisan 2015; Sarker et al. 2012) - that enable the co-creation of value in a service ecosystem.

Service exchanges involve the exchange of application of competencies by the members (Lusch and Nambisan 2015). Here, members mutually exchange their skills and capabilities for each other's benefits. Through this exchange, members jointly deliver value for the end customer. In the healthcare context, Srivastava \& Shainesh (2015) studied an ecosystem formed between a hospital and several rural vision centres to deliver eye care over telemedicine to rural areas in India. For each consultation, the hospital provided a specialist diagnosis which was then coupled with the patient examination provided by the rural vision centre. Together, each organization exchanged its application of competencies to facilitate effective delivery of telemedicine services.

Along with service exchange, organizations in an ecosystem also exchange resources which may augment value co-creation (Lusch and Nambisan 2015; Sarker et al. 2012; Srivastava and Shainesh 2015). We explicitly term this as resource exchanges to define the mutual actions taken by members (including keystone organization) in an ecosystem to access each other's resources. While some resources may be passive, resources such as knowledge and human capital when exchanged may be modified and become more idiosyncratic over time. For example, Sarker et al (2012) specifically examine the resource exchanges in the context of an ERP ecosystem which was formed between the ERP company and several partners. While the ERP company brought in resources such as brand and technology platform, partners brought in strong client relationships, deep industry knowledge and even development staff. These resources were then harnessed through appropriate exchange mechanisms to create more enhanced version of ERP product and hence more value for the clients. 
Third, since service ecosystems comprise of diverse organizations which have varied interests and incentives, these often require members to interact to develop shared purpose and coordinate their service and resource exchanges (Lusch and Nambisan 2015). We term these as relational exchanges to define the actual interactions and communications that steer the members towards shared purpose, aid in evaluation of future opportunities and resolution of conflicts (Lusch and Nambisan 2015). These also enable members to coordinate their resources and service exchanges by developing transparent rules of participation. As multiple organizations join the ecosystem, communication helps them to establish a common ground, enhance their relationships and share relevant information required to achieve shared goals (Neghina et al. 2014). Hence the frequency of such interactions and discussions among the members is important to the collaboration among the actors (Neghina et al. 2014). For example, Jha et al (2016) studied the growth of agricultural ecosystem formed to deliver services such as soil nutrient analysis to the farmers. For strengthening strategic planning and facilitating member interactions, farmer interest groups were formed which met at regular intervals to solve problems and plan activities such as procurement and marketing.

We next develop our hypotheses on the relationships between these three value cocreational exchanges and value to members in integrated care ecosystem.

\section{Service Exchanges and Value to Members}

Services are application of competencies (Lusch and Nambisan 2015) such as an organization applying its marketing skills to promote a product. Members engage in exchange of such services to jointly co-create value for the customers. In a typical telemedicine enabled ecosystem, service exchanges comprise of members (usually step down facilities such as community hospitals) exchanging their initial diagnosis of patients with the keystone organization (usually specialist hospitals) which then provides more in-depth knowledge and treatment recommendations. These exchanges enable delivery of a complete 
diagnostic consultation to the patient. These service exchanges are enabled through use of technology enabled service platforms such as remote patient monitoring, electronic health records and video-conferencing systems.

As members engage in exchange of services in an integrated manner with the keystone organization, value to members is enhanced on several fronts. First, quality of care is improved through greater continuity of care across multiple providers (Knight et al. 2016). With continued service exchanges, members become more efficient in handling cases (Hailey and Crowe 2000), respond more quickly to managing patient conditions (Schwamm 2014). Service exchanges can reduce the need for physical face-to-face visits, for example, for the elderly who are frail and find it challenging to physically go to hospitals (Mayhew 2009). This results in improved clinical outcomes or quality of care for members as their patients receive access to holistic care without undergoing the hassle of waiting in queues or further worsening of health conditions due to delay in care. Second, members satisfaction is increased as their confidence in use of technology and in managing different consultation cases grows with increased service exchanges (Becevic et al. 2015). Finally, costs such as transportation and staff time may be saved, as service exchanges also help members to achieve higher efficiency (Hailey and Crowe 2000). As members become efficient in delivering care over technology, physical visits to acute hospitals are avoided. We hence posit that

H1: More service exchange among members and key organization will be associated with increased value for members over time

Specifically, H1(a): More service exchanges among member and keystone organization will be associated with improved clinical outcomes for members over time 
H1(b): More service exchanges among member and keystone organization will be associated with improved satisfaction for members over time

H1(c): More service exchanges among member and keystone organization will be associated with cost effectiveness for members over time

\section{Resource exchanges and Value to members}

One motivation for members to join ecosystems is to access critical resources such as knowledge, technology or human capital which lie with other members or the keystone organization of the ecosystem (Lusch and Nambisan 2015). Members bring in resources that can be both complementary or supplementary to the existing ecosystem resources (Sarker et al. 2012). For example, in telemedicine-enabled integrated care ecosystems members often provide nursing staff, which when complemented with the specialist doctors of the keystone organization, result in delivery of the tele-consultation service. Members may also bring in supplementary resources such as beds or ambulances which may be utilized to achieve economies of scale. Over time these resources become co-specialized with changing customer needs (Dyer and Hatch 2006; Dyer and Singh 1998; Gnyawali and Madhavan 2001; Podolny and Page 1998) and hence their development is a crucial value co-creating mechanism (Dyer and Hatch 2006; Lusch and Nambisan 2015; Sarker et al. 2012).

Telemedicine-enabled ecosystems usually comprise of a keystone organization and several members who join to access specialist medical services (Paul 2006). Teleconsultation is facilitated by nurses and specialists who work collaboratively to examine a patient. Member usually provide the nursing staff. However, members' nurses are usually less qualified than those working in acute hospitals, and need to be clinically trained to perform basic assessment of the patient and to then communicate it to the specialist. Hence the keystone organization needs to transfer clinical knowledge and skills to members. This is 
usually done through clinical training programs which equip members' nursing staff with the necessary clinical knowledge and skills for preliminary diagnosis of patients.

Studies have found that increased nurse knowledge contributes to improved clinical outcomes through better clinical assessment (Knight et al. 2016) and reduction in the possibility of medication errors (Goran 2012). As nurses develop the ability to diagnose patients, members are in a better position to manage patients in-house and hence can avoid some unnecessary visits to the hospitals. Nurse training also leads to improved satisfaction as it uplifts their skillset and promotes critical thinking, communication and ability to use technology (Sevean et al. 2008). This increases nurse confidence in diagnostics as well as improved sense of professionalism (Toh et al. 2015). Finally, nurse training increases nursing home nurses' ability to diagnose patient conditions, and this can lead to reduced costs as patients get treated in a more timely manner, and unnecessary treatments and admissions to acute hospitals are avoided. Hence more resource exchanges among the member and the keystone organization over time will result in a clinically trained staff for members which in turn will help members derive value from the ecosystem. This set of arguments leads to the following hypotheses relating resource exchanges to value to members.

H2: More resource exchanges among member and keystone organization will be associated with increased value for members over time

Specifically,

H2(a): More resource exchanges among member and keystone organization will be associated over time with improved clinical outcomes for members over time

H2(b): More resource exchanges among member and keystone organization will be associated with improved satisfaction for members over time

H2(c): More resource exchanges among member and keystone organization will be associated with cost effectiveness for members over time 


\section{Impact of relational exchanges on service exchanges and resource exchanges}

While service exchanges and resource exchanges directly impact the value to members in an ecosystem, we argue that relational exchanges affect value to members through their influence on resource and service exchanges. Since relational exchanges are focused on developing necessary communication between members and the keystone organization, these allow for development of necessary mechanisms, rules and protocols around the ecosystem use (Lusch and Nambisan 2015).

Relational exchanges help members and the keystone organization to design effective ways to monitor and evaluate ongoing service exchanges to meet the shared value proposition (Stewart et al. 2013). For example, a poor fit between ecosystem's shared purpose and actual operational practices may result in less use of ecosystem services (Beech et al. 2013). Relational exchanges help define the roles and responsibilities shared by the members and the keystone organization. They also provide a platform for members to discuss issues encountered during actual service exchange. Together, through relational exchanges members and the keystone organization can engage in resolution of conflicts and coordination of service exchanges.

Telemedicine enabled ecosystems generally require members and the keystone organization to exchange services during the tele-consultation session. This requires nurses to exchange their first diagnosis with in-depth examination by the specialist. However, this service exchange requires coordination among the members and the keystone organization. Relational exchanges create an architecture of participation among the partners by defining roles, responsibilities and rules for exchange such as what information needs to be shared by the nurses and how the case report needs to be prepared. It also provides a platform for members to voice their concerns. For example, in case of an integrated care ecosystem, absence of relational exchanges was found to have negative effects on delivery of service as 
nurses had difficulty in communicating with other collaborators. This alienated them further feeling that their views are not been accounted for in the joint delivery (Beech et al. 2013). Since members and keystone organization have to jointly deliver the tele-consultation, we propose that:

\section{H3: More relational exchanges among members and keystone organization will be associated with more service exchanges over time}

With relational exchanges, members can also engage in strategic planning regarding the use of critical resources (Balasubramanian and Spurgeon 2012; Jha et al. 2016). For example, new ways can be devised to encourage knowledge sharing among members and the keystone organization. With transparency and protocols in place on resource use, members may be more willing to participate or increase their level in exchange of resources in the ecosystem over time.

Telemedicine enabled ecosystems generally require members and the keystone organization to engage in resource exchanges so that members are trained appropriately for tele-consultations. By engaging in relational exchanges, members can discuss issues they face during such trainings and can also decide on the allocation of nurse resources to these trainings. The keystone organization may also use these relational exchanges to communicate training requirements to the members that they may need to fulfill for tele-consultations. Relational exchanges hence increase transparency among both the members and the keystone organization which may increase their willingness to exchange resources. Hence we propose that,

H4: More relational exchanges among members and keystone organizations will be associated with more resource exchanges over time 
Figure 1 summarizes our proposed model for value co-creation exchanges and value to members in a telemedicine-enabled integrated care ecosystem

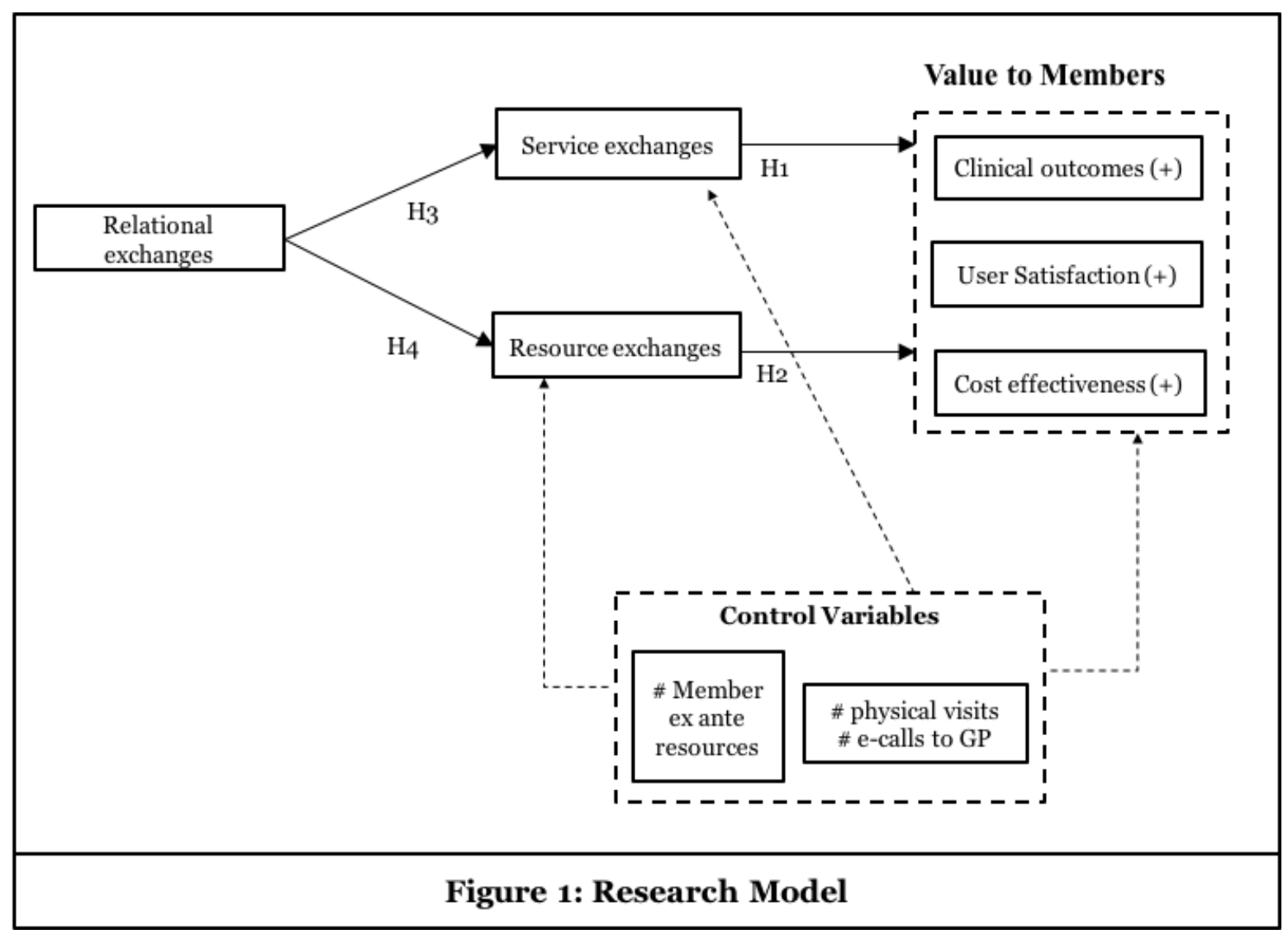

\section{METHODS AND MEASURES}

As we intended to explore the value to members when they engage in value cocreational exchanges in a service ecosystem, we selected a telemedicine enabled integrated care ecosystem as the research site for following specific reasons. First, the tele-geriatrics ecosystem we studied had successfully evolved for over five years now from its start in Dec 2010, with sustained partnerships with four members. This stable and longitudinal context provided a reliable context to test our hypothesis over time. Second, as the keystone organization had been monitoring and maintaining data rigorously for reporting purposes, we were able to access a rich dataset on not only value to members but also on efforts put in by members in developing service, resource and relationship exchanges with the keystone 
organization over time. With this rich dataset, we chose to conduct a panel data analysis to test our research model over time. Specifically, we used two-stage least square (2SLS) with fixed effects as it allowed us to control for unobserved member characteristics which do not change with time and may bias the coefficients as well as control for potential endogeneity concerns.

We now move on to provide a detailed overview of our research context followed by data collection and operationalization of variables. We then explain our proposed estimated model and methods.

\section{Research Context}

The research context is an integrated care tele-geriatrics ecosystem, which was initiated by a public funded hospital (Acute North) to provide specialist care to elderly patients in step down facilities (nursing homes). Acute North is a provider of community and specialist care services in the northern region of an Asian country. In line with the government's vision for delivering integrated care, Acute North's geriatrics department sought partnerships with nursing homes, which have a high density of elderly residents. These homes are non-profit voluntary organizations managing an average of 200 geriatrics residents per home. Each nursing home has between 15-17 nurses, and about 40 nursing aides all of whom are not trained to specifically manage the elderly health conditions. As these homes lack resources, they cannot afford specialist care and are dependent on acute hospitals for specialist services. The tele-geriatrics ecosystem was hence developed to reduce unnecessary visits to hospitals from these homes and improve care at homes.

A funding proposal was outlined to the Ministry of Health in 2010 to set up a videoconferencing system that would allow for specialist care through remote teleconsultations. By this, geriatricians in Acute North could communicate with patients without being physically present at the nursing home's premises. The nurses would act as hands for 
doctors to facilitate the diagnosis. As this required joint delivery of care, the proposal also included provision for nurse training programs to improve nurse's skillset for conducting clinical examinations with the specialist. The Ministry of Health assessed the proposal and approved funds for $\mathrm{S} \$ 2 \mathrm{~m}$ to be used over 5 years. As of year 2015 , the integrated care ecosystem comprised of Acute North and four partner nursing homes (NH1, NH2, NH3, NH4).

\section{Data Collection}

We constructed our panel dataset by collecting data from multiple sources. This included key performance indicator reports (monthly indicators on number of teleconsultation sessions, number of visits to acute hospitals, length of stay in hospitals, number of physical visits by doctors and nurse trainings), tele-consultation records (detailed information of each tele-consultation such as date of consult, start and end time of consult, user rating of the session), patient bills (bills incurred by patients in acute hospitals), presentation decks ( quarterly joint committee meetings), publically available annual reports of nursing homes ( number of beds, staff) and estimates of costs (prepared by Acute North and verified through an independent study). Apart from presentation decks, the rest of the datasets were quantitative in nature. The data was collected for each nursing home.

However, each nursing home had joined the tele-geriatrics ecosystem at a different time. For example, NH1 had joined in December, 2010 whereas NH3 came on board in December 2012. Hence for each nursing home we also collected pre tele-geriatrics data such as number of visits to acute hospitals, length of stay in hospitals, patient bills six months prior to their joining the ecosystem. This served as a baseline data for analysis. For example, for NH1 the baseline period was June 2010- November 2010. The baseline period was verified by Acute North for each nursing home. 
The uneven panels were hence harmonized by marking the joining month of each nursing home as the start of tele-consultation. For example, NH1 the first month of teleconsultation was December, 2010 and for NH3 it was marked as December 2012. A month dummy variable (month_dummy) was introduced and coded as 0 for pre-consultation period and $(1,2,3 \ldots \mathrm{n})$ for post consultation period. Hence for NH1, month_dummy was coded as 0 from June, 2010- November, 2010 and starting December 2010 till December 2015 it was coded as $(1,2,3 \ldots n)$.

With this, we created master dataset for each nursing home with monthly observations. However, data collected from multiple sources were on different timeframes. For example, performance indicator reports provided monthly indicators whereas, teleconsultation records and patient bills were on a daily basis, and joint committee meetings on quarterly basis. We therefore first matched the data to create monthly indicators in each category. For example, for the tele-consultation dataset, we prepared a monthly record for total time spent in consultation by aggregating the values from days for that month. These monthly values were added as new variables in the nursing home data file. Each observation was uniquely identified through year and month. Variables in columns indicated measures for that particular month. We then combined the four master files of nursing homes together. We introduced a dummy variable to uniquely identify each nursing homes and coded it as 1 for $\mathrm{NH} 1,2$ for $\mathrm{NH} 2,3$ for $\mathrm{NH} 3$ and 4 for $\mathrm{NH} 4$.

\section{Data Operationalization}

From the data collected, we operationalized the three value co-creating exchanges service, resource and relational as well as the value to members for each nursing home.

Service exchanges: These refer to the exchange of actual application of competencies by organizations (Lusch and Nambisan 2015). In this study, service exchange occurs during tele-consultations where the nurse presents each patient's case, doctor asks questions, nurse 
answers the questions (sometimes examining the patient further), and then the doctor provides the diagnosis and prescriptions. As this service exchange was necessary for each patient consulted, we operationalized service exchange as the number of patients treated using tele-consultation each month (ServiceX). This indicated a key aspect of how both the keystone organization and members exchange their respective competencies to deliver a service to the end customer.

Resource exchanges: Resource exchanges specify the mutual and reciprocal actions taken by organizations to harness each other's resources for joint delivery of services. In this study, a critical resource exchange was the members' provision of nurses and the keystone organization's transfer of clinical knowledge to nursing homes in order to jointly conduct tele-consultation. This resource exchange was primarily done via training sessions, conducted by trainers employed by Acute North, using material developed by Acute North. These training sessions focused on developing nurse's critical thinking skills so that they can identify patient symptoms, diagnose problems through clinical photographs, develop communication skills with doctors and learn nursing interventions. We therefore operationalized resource exchange as the number of nurses trained as a percentage of total nursing staff in nursing homes in each month (ResourceX).

Relational exchanges: These refer to the actual communications among the members and the keystone organization on various issues such as development of a shared purpose, sharing of opportunities and resolution of disputes during implementations (Lusch and Nambisan 2015; Sarker et al. 2012). With discussions of various issues, members and the keystone organization can develop clarity and work collaboratively in the ecosystem. One key forum for relational exchanges in our study were the joint committee meetings (held quarterly), chaired by the keystone organization, and attended by representatives from all the member nursing homes. At these meetings, issues important to the functioning and evolution 
of the integrated care ecosystem were raised and discussed. Nursing homes participated in these meetings to discuss issues such as nurse trainings, KPI numbers or technology infrastructure.

We operationalized relational exchanges as the total number of issues discussed by a nursing home at each committee meeting. Our iterative coding of the presentation decks of the quarterly committee meetings led us to develop seven domains that required member and keystone organization's inputs and decision-making in the tele-geriatrics ecosystem. These domains were organizational, technology, platform use, future planning, goals, training and setting processes. We coded each instance of nursing homes' participation in each of these domains as 1. For example, if NH1 participated in KPI discussions, it was coded as 1 for NH1. However, if any nursing home did not participate in the meeting discussion in any of the domains, it was coded as 0 . Discussions relevant to all nursing homes, for example, on shared purpose of tele-geriatrics, were coded as 1 for all nursing homes. Hence for each nursing home, we operationalized relational exchanges as the total number of discussions participated in by the nursing home (RelationalX).

\section{Value to Members}

From prior research, three key aspects of value from integrated care were identifiedclinical outcomes, user satisfaction, and cost effectiveness (Balasubramanian and Spurgeon 2012; Busse and Stahl 2014; Chae et al. 2001; Goran 2012; Greaves et al. 2013; Jacobs et al. 2012; Larcher et al. 2003; Mayhew 2009; McLean et al. 2011; Sandoval Garrido et al. 2014).

Clinical outcomes: Clinical outcomes are an indicator of improvement of care for patients. Prior studies measure this improvement either through generic measures such as reduction in hospitalization and mortality rate (Darkins et al. 2008; Goran 2012; Weinberger et al. 1996) or specific measures such as number of falls, pressure ulcers, pain severity, 
depression, dehydration levels (Balasubramanian and Spurgeon 2012; Goran 2012; Greaves et al. 2013; Sandoval Garrido et al. 2014).

In this study, nursing homes are step down facilities managing chronically ill patients, lacking skills required to manage patients in-house. Hence patients are often transported via ambulance to the nearest acute hospital for specialist consultation. However, often it was found that patients were sent back to nursing homes after waiting for long hours as their conditions did not warrant hospitalization. This was traumatic for the elderly patients as they had to wait for hours in the unfamiliar and stressful environment of the A\&E department, only to be assessed as not needing hospitalization and to return to the nursing home with prescribed medication. Also, staying for longer hours in hospitals made them vulnerable to further infections. Reducing these unnecessary visits to the hospitals was one of the key goals of the tele-geriatrics ecosystem. With tele-geriatrics, elderly patients could avoid the stress of waiting and risk of further catching infections in an unfamiliar hospital environment. As noted by the Nurse Manager in NH1,

"But the hassle of transport, hassle of going through that admission process, sometimes, they wait in A\&E for days before getting a bed due to bed crunch in the hospitals... so why put them through all this, especially when we are talking about quality of life...Tele $G$ comes and helps us with lot of this, talks about dignity of dying”- NH1, Manager

Similarly, if nursing homes benefited from the improvement in quality of care, it also reduced the burden on Acute North's resources as lesser patients are referred to the hospitals. As noted by the senior consultant at Acute North

"The goal to the hospital administration is to reduce hospital admissions"- Senior Consultant, Acute North 
We hence operationalized clinical outcomes as number of visits to acute hospitals each month. We could not include specific clinical measures such as number of falls, pressure ulcers as Acute North had not been monitoring these during course of our research.

User Satisfaction: Second, the use of telemedicine also provides value to members by increasing patient and user satisfaction (Balasubramanian and Spurgeon 2012; Becevic et al. 2015; Chae et al. 2001; Jacobs et al. 2012; Larcher et al. 2003). Hence, measures such as employee satisfaction or clinician satisfaction from tele-consult session have often been used to evaluate value to members from the use of telemedicine.

In this study, one motivation for nursing homes to join the tele-geriatrics ecosystem was to improve care for their patients by providing access to specialist services. Since most of the patients suffered from chronic illness like dementia or behavioral issues which could be assessed without touching the patient, tele-consultation provided an equivalent alternative to physical consultations. However, it was important that a tele-consultation be conducted smoothly over technology without any technical or process issues so that it could be at par to a physical visit. If not, this could result in lower satisfaction for members as they would face the hassle of interrupted communication with the specialists. As noted by the nurse director at NH3,

"We find it very irritating as we cannot hear them. It's not stable. Now they have gone much more changes. It has become more stable now."- Nurse Director, NH3

A similar concern was raised by the users at Acute North on process of tele-consultation "Nurses way of case presentation is a problem like accent becomes an issue. If they are really trained then they run it on their own... else doctors have to call clinical educators if they don't trust the nurses."- Operations Executive, Acute North Therefore, in this study, we measure satisfaction from the overall experience of members with a tele-consultation session. After each tele-consultation session, nurses 
together with the doctors, rated the session on issues arising from technology, process or person. A session was rated as an ideal session when no such issue was encountered during the consultation else was rated as non-ideal. In case the tele-consultation session was dismissed, it was labeled aborted. An ideal session indicated satisfaction and value to members as they could provide uninterrupted care to their patients. These ratings were used to operationalize user satisfaction. For each session, we coded Ideal session as 1 , Non ideal and aborted as 0 . Average of these ratings were taken as an aggregate for each month.

Cost effectiveness: With telemedicine, members also seek cost effectiveness by avoiding clinical and administrative costs and providing timely interventions (Balasubramanian and Spurgeon 2012; Franzini et al. 2011; Rojas and Gagnon 2008; Whittaker and Wade 2014). Indicators such as total healthcare cost, cost per patient or hospitalization costs have often been used extensively to evaluate cost associated with telemedicine (Rojas and Gagnon 2008).

In this study, to evaluate cost effectiveness, we estimated two types of costs incurred to nursing homes - outpatient costs and inpatient costs. First, we estimated outpatient costs which are costs incurred when nursing home patients visit acute hospitals to see specialists. For every month, using the patient billing data, total number of outpatients were flagged. A patient was marked an outpatient when there were no ward charges in the bill and discharge date was same as the admission date. For each outpatient visit, we applied an estimate of S\$ 286 per hour. These estimates were provided by Acute North (calculated by estimating cost and time of specialist, ambulance and staff involved in each outpatient visit) after validation at Common Wealth Fund Symposium 2015 and Ministry of Health. Each outpatient visit lasted for two hours.

Second, we estimated, inpatient costs which are costs incurred to nursing homes when patients are admitted to acute hospitals. While some of these admissions are planned for e.g. 
a surgery to remove cataracts or fix a fracture, telemedicine is aimed to reduce the additional ad-hoc costs from unplanned admissions as planned admissions are out of its purview. We hence, used the number of unplanned admissions in each month and average length of stay (inpatient days) of patients in a month to compute total length of stay of patients in acute hospitals. On this value, we applied an estimate of $\$ \$ 503$ per day. This estimate was provided by the Ministry of Health and validated by Acute North.

We measured cost effectiveness by calculating the medical cost incurred when nursing home patients access acute hospital services. This was computed as the total of outpatient and inpatient costs (Medical cost). Citing the expected cost savings from the ecosystem, the nurse director at one of the nursing homes noted that,

"We know the benefit of this. There is another mode of residents can be seen by doctors. Most important this project is free... Two hours' visit can cost about 450\$600\$."- Nurse Director, NH3

Likewise, from Acute North's end, a similar expectation was noted by the Senior operations manager,

"Then we also made sure that the RF funding is $100 \%$...the doctors' time at NH is all paid for by the project. So it is money savings for the NH when they join the project as they don't have to pay for the geriatric services. Tele G is free."

\section{Control Variables}

We control for two sets of variables that can potentially affect both exchanges and value to members. First are the member ex ante resources. In this study, we found that nursing homes differed in their portfolio of resources such as staff, beds and residents. If some nursing homes are well resourced, it is likely that it will affect their exchanges with Acute North. For example, if nursing homes have limited staff nurses, it may impact their resource exchanges as they may have less staff available for training. Also, presence of these resources may 
affect the value to members. For example, number of beds and staff in a nursing home may affect its use of tele-consultation. If a nursing home has more staff, they are likely to take better care of the patients in the home, which in turn will affect the value to members. We hence operationalized member ex ante resources as number of residents (nh_residents), staff (nh_staff) and beds (nh_beds) in nursing homes. Second, we also controlled for alternative options available to nursing homes to access care. Apart from tele-consultations, nursing homes could also access specialist care by physical consultations where geriatrics specialists from Acute North visit nursing homes. We controlled for both number of physical visits and the number of patients seen in those visits. In addition, nursing homes could make e-calls to general practitioners (GP) to discuss resident cases. We control for the total e-calls to GP as it is likely these affect value to members. Last, we also control for unplanned admissions as a percentage of total residents when evaluating cost effectiveness.

Table 1 and 2 provide summary of descriptive statistics and correlations. 


\begin{tabular}{|l|l|l|l|}
\hline \multicolumn{2}{|l|}{ Table1: Summary of descriptive statistics } & Mean & S.D. \\
\hline Variable name & Variable Description & & \\
\hline & & 4.668 & 4.049 \\
\hline ServiceX & Patients consulted per NH per month & 0.156 & 0.209 \\
\hline ResourceX & Percentage of staff trained per NH per month & 3.716 \\
\hline RelationalX & $\begin{array}{l}\text { Participation of each NH in quarterly joint } \\
\text { meetings }\end{array}$ & 8.043 & 5.339 \\
\hline Clinical outcomes & $\begin{array}{l}\text { Number of visits to Acute Hospitals each } \\
\text { month }\end{array}$ & 7.9565 & .4177 \\
\hline User satisfaction & Overall rating of the tele-consultation session & .4617 & 22537.73 \\
\hline Medical Cost & Total inpatient and outpatient costs & 35453.75 & \\
\hline Controls & & \multicolumn{2}{l|}{} \\
\hline Phyvisits & Number of physical visits by specialists & 0.829 & 0.820 \\
\hline Visits_pat & $\begin{array}{l}\text { Number of patients seen in these physical } \\
\text { visits }\end{array}$ & 4.725 & 5.862 \\
\hline Gp_calls & Number of calls to general practitioner & 0.168 & 0.608 \\
\hline Nh_residents & Number of Residents per NH & 168.048 & 45.513 \\
\hline Nh_staff & Number of staff per NH & 78.184 & 29.064 \\
\hline Nh_beds & Number of beds per NH & 187.163 & 47.846 \\
\hline Unplanned_adm & Percentage of unplanned admissions & 0.03918 & 0.0242 \\
\hline
\end{tabular}

Note: Panel data from June 2010 to December 2015 for four nursing homes Observations $(n=184)$ 


\begin{tabular}{|c|c|c|c|c|c|c|c|c|c|c|c|c|c|c|}
\hline & & (1) & (2) & (3) & (4) & (5) & (6) & (7) & (8) & (9) & (10) & (11) & (12) & (13) \\
\hline 1 & ServiceX & 1.000 & & & & & & & & & & & & \\
\hline 2 & ResourceX & \begin{tabular}{|l|}
-0.003 \\
\end{tabular} & 1.000 & & & & & & & & & & & \\
\hline 3 & RelationalX & 0.019 & 0.252 & 1.000 & & & & & & & & & & \\
\hline 4 & Clinical Outcomes & 0.001 & 0.064 & 0.023 & 1.000 & & & & & & & & & \\
\hline 5 & User Satisfaction & 0.475 & -0.207 & 0.015 & 0.108 & 1.000 & & & & & & & & \\
\hline 6 & Medical Cost & -0.101 & 0.227 & 0.108 & 0.798 & 0.023 & 1.000 & & & & & & & \\
\hline 7 & Phyvisits & 0.160 & 0.025 & 0.019 & -0.347 & -0.080 & -0.368 & 1.000 & & & & & & \\
\hline 8 & Visits_pat & 0.110 & 0.053 & 0.079 & -0.241 & -0.096 & -0.254 & 0.824 & 1.000 & & & & & \\
\hline 9 & Gp_calls & -0.041 & 0.165 & 0.242 & $\begin{array}{l}0.021 \\
9\end{array}$ & $\begin{array}{l}0.001 \\
6\end{array}$ & 0.189 & -0.077 & -0.178 & 1.000 & & & & \\
\hline 10 & Nh_residents & 0.332 & -0.210 & -0.140 & $\begin{array}{l}0.071 \\
2\end{array}$ & $\begin{array}{l}0.433 \\
2\end{array}$ & 0.035 & -0.024 & 0.051 & -0.016 & 1.000 & & & \\
\hline 11 & Nh_staff & 0.114 & -0.170 & 0.017 & -0.201 & -0.246 & -0.173 & 0.116 & 0.088 & 0.055 & 0.033 & 1.000 & & \\
\hline 12 & Nh_beds & 0.341 & -0.115 & -0.010 & $\begin{array}{l}0.064 \\
5\end{array}$ & 0.447 & $\begin{array}{l}0.057 \\
9\end{array}$ & -0.025 & 0.036 & -0.016 & 0.821 & 0.127 & 1.000 & \\
\hline 13 & Unplanned_adm & -0.047 & 0.207 & 0.204 & 0.864 & -0.008 & 0.761 & -0.250 & -0.159 & -0.025 & -0.157 & -0.208 & -0.105 & 1.000 \\
\hline
\end{tabular}




\section{Model Estimation}

To test our hypothesis, we build our model by specifying member outcomes as a function of two constructs- resource exchanges and service exchanges in this equation where $\mathrm{S}$ is service exchange, $\mathrm{R}$ is resource exchanges, and $\mathrm{C}$ is the list of exogenous controls.

$$
O=\mathrm{f}(S, R, C)
$$

Further, we define resource and service exchanges as a function of relational exchanges (I) and controls (X)

$$
\begin{aligned}
& S=\mathrm{f}(I, X) \\
& R=\mathrm{f}(I, X)
\end{aligned}
$$

Specifically, we estimate the following equations. For service and resource exchanges we use 4(a) and 4(b)

$$
\begin{aligned}
& S_{i t}=\delta_{0}+\delta_{1} I_{i t}+\delta_{2} X_{i t} \delta_{6}+\omega_{i}+\epsilon_{i t} \\
& R_{i t}=\gamma_{0}+\gamma_{1} I_{i t}+\gamma_{2} X_{i t}+v_{i}+\xi_{i t}
\end{aligned}
$$

where $v_{i} \& \omega_{i}$ are error idiosyncratic to nursing homes, $\xi_{i t} \& \epsilon_{\text {it }}$ are stochastic error to nursing home and time and $\gamma \& \delta$ are parameters.

We next estimate the value to members in equation (5)

$$
O_{i t}=\beta_{0}+\beta_{1} S_{i t}+\beta_{2} R_{i t}+\beta_{3} C_{i t}++\mu_{i}+\varepsilon_{i t}
$$

where $O_{i t}$ is the value for nursing home $i$ at time $t, S_{i t}$ are service exchanges for nursing home $i$ at time $t, R_{i t}$ are resource exchanges for nursing home $i$ at time $t, \beta$ is the parameter, $\mu_{i}$ is error idiosyncratic to nursing homes and $\varepsilon_{i t}$ is stochastic error to nursing home and time. 
Equation (5) which estimates the value to members has two endogenous variablesresource exchanges and service exchanges. A variable is endogenous when it is function of other variables present in the system. In this case if we estimate equation (5) the coefficients of endogenous variables will be unbiased only if error term $\varepsilon_{i t}$ is uncorrelated with $\xi_{i t} \& \epsilon_{i t}$. If these errors are correlated it implies that outcome variable will be correlated with the error term and hence estimates of $\beta_{1}$ and $\beta_{2}$ will be biased.

One way to deal with endogeneity is to use the predicted values of equations 4(a) and 4(b) rather than actual values while estimating equation (5). By using predicted values of equations, error terms $\xi_{i t} \& \epsilon_{i t}$ will be removed from the model in equation (5). Second, we also use one additional instrumental variable which is correlated with the service exchanges but not with the value to members. For nursing homes, the likelihood of engaging in service exchanges is dependent on the number of trained staff they have. We regress the service exchanges on the number of trained staff and store the residual as the instrumental variable. The rationale for this variable as instrumental variable is that it measures the natural propensity of nursing homes to engage in service exchanges. While this is correlated with service exchanges, it is unlikely to be correlated with the value to members.

We hence use 2SLS with fixed effects to estimate the model. In the first stage, the predicted values are generated for equations 4(a) and 4(b). Then in the second stage, these values are used to estimate the outcomes. Using a fixed effects analysis helps us to remove the unobserved nursing home characteristics which do not change with time and may bias the coefficients $^{1}$.

\footnotetext{
${ }^{1}$ We conducted further robustness checks for serial auto-correlation, over-identification restrictions (Hansen J tests), interaction effects. The findings reported are robust to these tests.
} 
Multicollinearity is not a significant issue as VIF is less than 6.2 for all independent variables. (VIF less than five or ten is often used as a threshold to test multicollinearity; (Ménard 1995; Myers 1990). We also test for heteroskedasticity using Wald test (Prob $>$ chi2 $=0.000$ ) suggesting presence of heteroskedasticity. Hence robust standard errors were calculated. We also test for serial correlation. Usually it is not a problem in small panels but Langram- multiplier test is performed which confirms that serial correlation is not present $(\mathrm{F}(1,3)=1.03$, prob $>\mathrm{F}=$ $0.3849)$.

\section{RESULTS}

First, we examine how service and resource exchanges effect value to members (Hypothesis 1 and 2). The results of two stage least squares with fixed effects are shown in Table 3(a), (b) and (c). Table 3(a) and 3(b) shows the results for clinical outcomes and user satisfaction. Table 3(c) shows the results for cost effectiveness.

$\mathrm{H} 1 \mathrm{a}$ and H1b suggest that more service exchanges are associated with improved clinical outcomes and improved user satisfaction respectively over time. While our results do not support H1a, we find support for $\mathrm{H} 1 \mathrm{~b}\left(\beta_{1}=0.05308, p\right.$-value $\left.<0.01\right)$ suggesting that user satisfaction has improved with more service exchanges over time $\left(\beta_{\text {month_dummy }}=0.01034, p\right.$-value $\left.<0.01\right)$. H1c suggests that more service exchanges are associated with decrease in costs over time. We examine the cost effectiveness as a sum total of inpatient and outpatient costs (Medical cost). The findings support H1c $\left(\beta_{1}=-668.449, p\right.$-value $\left.<0.05\right)$ suggesting that with service exchanges overall inpatient and outpatient costs have decreased.

$\mathrm{H} 2 \mathrm{a}$ and $\mathrm{H} 2 \mathrm{~b}$ suggest that more resource exchanges are associated with improved clinical outcomes and improved user satisfaction respectively over time. The findings support $\mathrm{H} 2 \mathrm{a}\left(\beta_{2}=\right.$ 
$-2.7789, p$-value $<0.05)$ suggesting that number of visits to acute hospitals has reduced with more resource exchanges over time $\left(\beta_{\text {month_dummy }}=-0.0957, p\right.$-value $\left.<0.01\right)$. However, we did not find support for $\mathrm{H} 2 \mathrm{~b}$. We also did not find for $\mathrm{H} 2 \mathrm{c}$ which suggests that more resource exchanges are associated with decrease in costs over time.

Last, the results of fixed effects estimation for the second set of hypotheses ( $\mathrm{H} 3$ and $\mathrm{H} 4$ ) are presented in Table 4. The results support $\mathrm{H} 4\left(\gamma_{1}=0.013622, p\right.$-value $\left.<0.05\right)$ suggesting that an increase in relational exchanges between members and the keystone organization is associated with greater resource exchanges. The time dummy variable however is negative $\left(\gamma_{\text {month_dummy }}=-\right.$ $0.0050, p$-value $<0.05$ ) suggesting that over time less nursing staff has been undergoing training. We, however did not find support for H3. Table 5 provides a summary of our findings.

Table 3 (a): Controlling for endogenity (H1 \& H2)- Clinical outcomes

\begin{tabular}{|l|l|l|l|l|}
\hline & \multicolumn{2}{|l|}{ Clinical outcomes } & \multicolumn{2}{l|}{} \\
& Coefficient & Std. error & \multicolumn{2}{l|}{ 95\% confidence interval } \\
\hline ServiceX & 0.02962 & 0.06677 & -.1012512 & 0.16049 \\
\hline ResourceX & $-2.7789^{* *}$ & 1.1694 & -5.071029 & -0.48694 \\
\hline Month_dummy & $-0.0957^{* * *}$ & 0.01976 & -0.1345 & -0.0570 \\
\hline Phyvisits_nbr & 0.23181 & 0.43390 & -0.6186 & 1.0822 \\
\hline Visit_pat & -0.0051 & 0.0631 & -0.1289 & 0.11862 \\
\hline Gp_calls & 0.06486 & 0.3062 & -0.53546 & 0.66519 \\
\hline NH_residents & $0.03799^{* *}$ & 0.01600 & 0.00661 & 0.0693 \\
\hline NH_staff & $-0.1258^{* * *}$ & 0.0252 & -0.1752 & -0.0764 \\
\hline NH_beds & 0.0093 & 0.01320 & -0.0165 & 0.03519 \\
\hline F value (p $>0.0005)$ & 5.78 & & & \\
\hline R-square (total) & 0.2106 & & & \\
\hline Note & & & & \\
\hline
\end{tabular}

Note ${ }^{* * *}$ represents p-value $<0.01 ; * *$ represents $\mathrm{p}$-value $<0.05 ; *$ represents $\mathrm{p}$-value $<0.1$. 
Table 3 (b): Controlling for endogenity (H1 \& H2)- User satisfaction

\begin{tabular}{|l|l|l|l|l|}
\hline & \multicolumn{2}{|l|}{ User satisfaction } & \multicolumn{2}{l|}{} \\
& Coefficient & Std. error & 95\% confidence intervals \\
\hline ServiceX & $0.05308^{* * *}$ & 0.0078 & 0.03762 & 0.06854 \\
\hline ResourceX & -0.18898 & .14413 & -0.47147 & 0.09351 \\
\hline Month_dummy & $0.01034^{* * *}$ & 0.0021 & 0.00617 & 0.014512 \\
\hline Phyvisits_nbr & 0.0409 & 0.0507 & -0.05843 & 0.014036 \\
\hline Visit_pat & -0.0064 & 0.0060 & -0.018324 & 0.00542 \\
\hline Gp_calls & 0.5406 & 0.3565 & -0.015811 & 0.123935 \\
\hline NH_residents & 0.0008 & 0.0020 & -0.00484 & 0.00322 \\
\hline NH_staff & $-0.0058^{* *}$ & 0.0025 & -0.01080 & -0.00088 \\
\hline NH_beds & 0.0034 & 0.0017 & 0.00056 & 0.006769 \\
\hline F value (p>0.0005) & 22.51 & & & \\
\hline R-square (total) & 0.4186 & & & \\
\hline
\end{tabular}

Note $* * *$ represents $\mathrm{p}$-value $<0.01 ; * *$ represents $\mathrm{p}$-value $<0.05 ; *$ represents $\mathrm{p}$-value $<0.1$.

Table 3 (c): Controlling for endogenity (H1 \& H2)- Cost effectiveness

\begin{tabular}{|l|l|l|l|l|}
\hline \multirow{2}{*}{} & \multicolumn{2}{|l|}{ Medical Cost } & \multicolumn{2}{l|}{} \\
\cline { 2 - 5 } & Coefficient & Std. error & \multicolumn{2}{l|}{$95 \%$ confidence interval } \\
\hline ServiceX & $-668.449^{* *}$ & 337.9588 & -1330.837 & -6.0628 \\
\hline ResourceX & 5447.5 & 3590.363 & -1590.658 & 12485.66 \\
\hline Month_dummy & -59.7399 & 121.909 & -298.6789 & 179.199 \\
\hline Phyvisits_nbr & -745.9284 & 2419.587 & -5488.232 & 3996.375 \\
\hline Visit_pat & 19.3540 & 274.981 & -523.5205 & 562.228 \\
\hline Gp_calls & $5841.90^{* *}$ & 2324.04 & 1286.864 & 10396.95 \\
\hline NH_residents & $144.9362^{* *}$ & 61.6995 & 24.0073 & 265.865 \\
\hline NH_staff & -106.192 & 102.672 & -307.4264 & 95.0415 \\
\hline NH_beds & 57.6992 & 40.6433 & -21.9602 & 137.3588 \\
\hline Unplanned_adm & $587098.7^{* * *}$ & 74421.7 & 441234.7 & 732962.7 \\
\hline F value (p $>0.01)$ & 16.05 & & & \\
\hline R-square (total) & 0.5321 & & & \\
\hline & & & & \\
\hline
\end{tabular}

Note $* * *$ represents $\mathrm{p}$-value $<0.01 ; * *$ represents $\mathrm{p}$-value $<0.05 ; *$ represents $\mathrm{p}$-value $<0.1$. 
Table 4: Fixed effects Estimation H3 \& H4

\begin{tabular}{|l|l|l|l|l|}
\hline \multirow{2}{*}{} & \multicolumn{2}{|l|}{ Service exchanges (H3) } & \multicolumn{2}{l|}{ Resource exchanges (H4) } \\
\cline { 2 - 5 } & Coefficient & Std. error & Coefficient & Std. error \\
\hline RelationalX & 0.18853 & 0.11918 & $0.013622^{* *}$ & 0.00355 \\
\hline NH_beds & & & & \\
\hline NH_staff & 0.03727 & 0.02457 & 0.00199 & 0.00087 \\
\hline NH_residents & 0.01343 & 0.03138 & -0.0045 & 0.00096 \\
\hline GP_calls & 0.02062 & 0.03095 & -0.0028 & 0.00166 \\
\hline Month_dummy & 0.04165 & 0.37969 & 0.03549 & 0.03163 \\
\hline Constant & -0.03883 & 0.01910 & $-0.0050^{*}$ & 0.00183 \\
\hline R-square (within) & $3.94320^{* *}$ & 1.02611 & $0.14565^{* *}$ & 0.33435 \\
\hline R-square (total) & 0.1527 & & 0.2410 & \\
\hline Note: & 0.0829 & & 0.1982 & \\
\hline
\end{tabular}

Note: $* * *$ represents $\mathrm{p}$-value $<0.01 ; * *$ represents $\mathrm{p}$-value $<0.05 ; *$ represents $\mathrm{p}$-value $<0.1$.

Table 5: Summary of Hypothesis

\begin{tabular}{|l|l|}
\hline Hypothesis & Result \\
\hline $\begin{array}{l}\text { H1(a): More service exchanges among member and keystone organization will } \\
\text { be associated with improved clinical outcomes for members over time }\end{array}$ & Not supported \\
\hline $\begin{array}{l}\text { H1(b): More service exchanges among member and keystone organization will } \\
\text { be associated with improved satisfaction for members over time }\end{array}$ & Supported \\
\hline $\begin{array}{l}\text { H1(c): More service exchanges among member and keystone organization will } \\
\text { be associated with cost effectiveness for members over time }\end{array}$ & Supported \\
\hline $\begin{array}{l}\text { H2(a): More resource exchanges among member and keystone organization } \\
\text { will be associated over time with improved clinical outcomes for members over } \\
\text { time }\end{array}$ & Supported \\
\hline $\begin{array}{l}\text { H2(b): More resource exchanges among member and keystone organization } \\
\text { will be associated with improved satisfaction for members over time }\end{array}$ & Not supported \\
\hline $\begin{array}{l}\text { H2(c): More resource exchanges among member and keystone organization will } \\
\text { be associated with cost effectiveness for members over time }\end{array}$ & Not supported \\
\hline $\begin{array}{l}\text { H3: More relational exchanges among members and keystone organizations } \\
\text { will be associated with more service exchanges over time }\end{array}$ & Not supported \\
\hline $\begin{array}{l}\text { H4: More relational exchanges among members and keystone organizations } \\
\text { will be associated with more resource exchanges over time }\end{array}$ & Supported \\
\hline
\end{tabular}




\section{DISCUSSION}

In this study, we examine the value to members in a service ecosystem when they participate in value co-creational exchanges. We test our hypothesis in an integrated care context and measure both economic (cost effectiveness) and non-economic (improved quality of care and user satisfaction) value to members when they engage in three value co-creational exchanges- service, resource and relational. While prior research has mostly emphasized on the role of resource exchanges in value co-creation (Sarker et al. 2012; Srivastava and Shainesh 2015), our findings suggest that in addition to resource exchanges, service exchanges and relational exchanges also affect the value to members. We now discuss here in detail our results and how value to member is affected by the value co-creational exchanges. Figure 2 summarizes our proposed model and results.

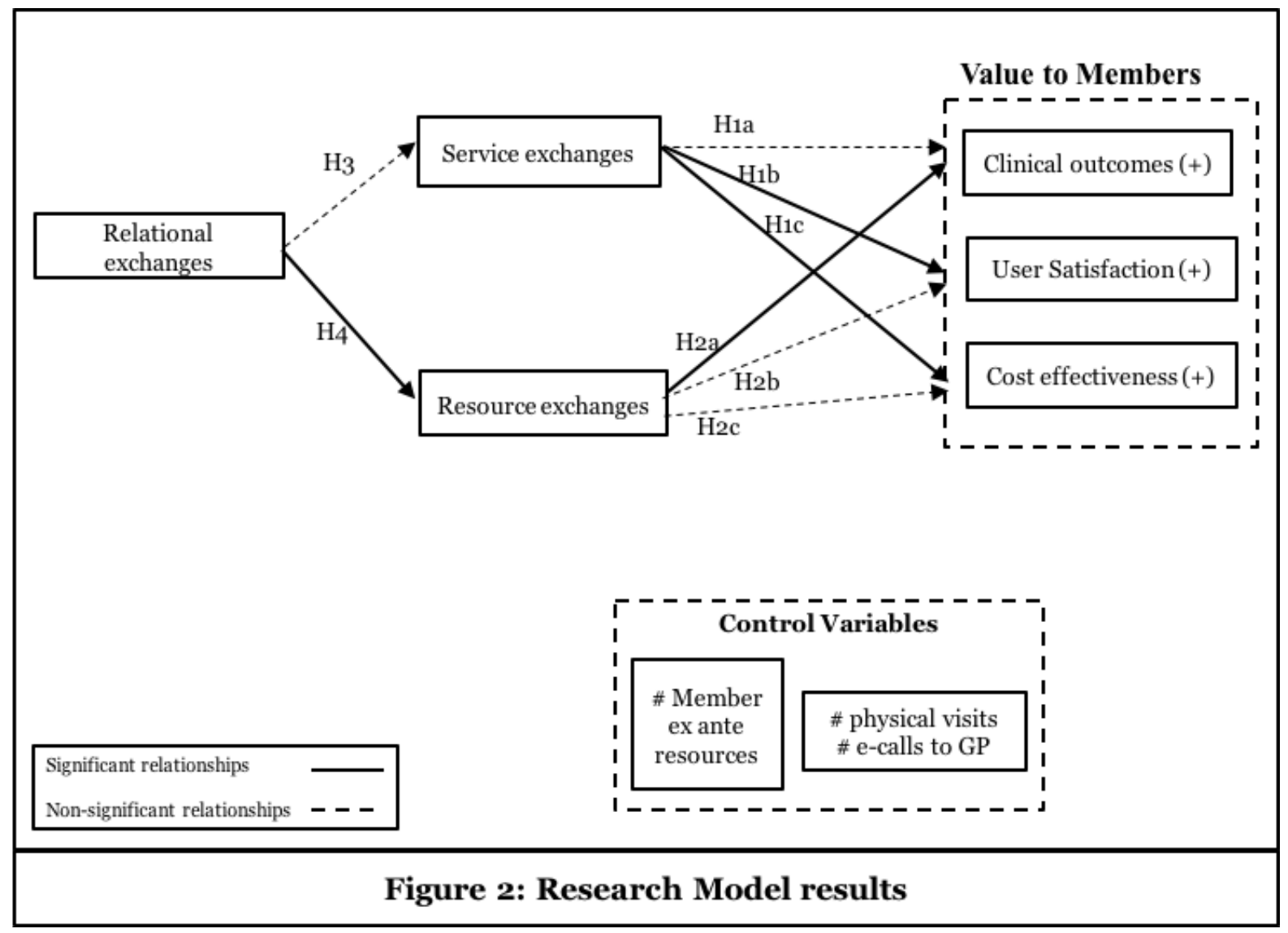




\section{Clinical Outcomes}

In Hypothesis 1(a) and 2(a) we argue that more service and resource exchanges will be associated with improved clinical outcomes (measured as visits to acute hospital). Consistent with our hypothesis $\mathrm{H} 2(\mathrm{a})$, we found that with increased resource exchange, in terms of having more trained nurses, clinical outcomes were improved for nursing homes. Prior to joining telegeriatrics, members relied extensively on acute hospitals when their patients fell ill, as their nurses lacked clinical skills to assess patient conditions. In fact, many of these patients were often discharged from acute hospitals after simple antibiotic treatments as their conditions did not warrant hospitalization. With tele-geriatrics, nurses were now specifically trained by Acute North on how to assess patient symptoms and then decide whether to send the patient to acute hospital or not. With better training, nursing homes were able to avoid unnecessary visits to the hospitals. As noted by the nurse manager at NH2

"They are much more confident in making judgments. They know whether they should send the patient to hospital or wait for assessment."

In H1(a) we argued that with more service exchanges, clinical outcomes would be improved for nursing homes since patients could access specialist consultations through telemedicine which would avoid visits to acute hospitals. As noted earlier, transfers to hospitals were traumatic for the elderly patients as they had to wait for long hours to access care. With their frail conditions, they were also at higher risk of contracting infections in hospitals. With telemedicine, patients could access timely care in-house which would reduce their stress of visiting hospitals and eventually improve quality of care. However, we did not find support for this hypothesis. 
On further consideration of the data, we realized the relationship between service exchange (i.e. patients seen through tele-consult sessions) and clinical outcomes, as measured by number of hospital admissions may not be as straightforward. Prior to tele-geriatrics, a range of patients were being referred to acute hospitals as nurses were not able to make an informed judgment of their medical conditions. However, with tele-geriatrics, nurses were now being specifically trained to monitor patient conditions and perform basic interventions. With quicker response to patient conditions, nurses now may be selecting patients for tele-consults whose symptoms might have been missed by the nursing staff before. These cases tend to be the ones that nurses cannot easily manage themselves. As noted by the nurse manager in NH2-

"We see changes in health and behavior daily. Doctor treat the patient that need more attention."

These more difficult cases may eventually be either cleared by the specialist during teleconsult or referred to acute hospital for further follow up. Hence, more tele-consults need not necessarily result in a reduction in hospital admissions.

Taken together, the findings for $\mathrm{H} 1 \mathrm{a}$ and $\mathrm{H} 2 \mathrm{a}$ suggest that, while tele-consultations did provide a timely access to care to nursing homes resulting in improved diagnosis, it was not necessarily being reflected in visits to hospitals. Possibly, the effect of consultations on clinical outcomes could be better examined through more specific measures of quality of care such as reduction in pain or healing time of ulcers.

\section{User satisfaction}

In Hypothesis 1(b) and 2(b) we argue that more service and resource exchanges will be associated with improved member satisfaction. Consistent with $\mathrm{H} 1(\mathrm{~b})$, we found that member's satisfaction improved with more service exchanges. First, with increased tele-consultation 
sessions, nurses increased their confidence in use of technology and managing different cases. With more service exchanges, nurses' confidence of conducting assessments increases as they learn from the specialists which in turn increases their satisfaction. As noted by the nurse director at $\mathrm{NH} 3$,

"They are confident and much more comfortable talking with doctors."

Second, an ideal telemedicine session (where there is no person, technical or process issue) also provides a satisfactory experience for members as they are able to access specialist care with minimum hassles.

In hypothesis 2(b) we also expected that more resource exchanges will result in improved satisfaction for members as it uplifts their skills and ability to assess their residents. However, we did not find support for this argument. Our further analysis of the case revealed that while resource exchanges are critical for members to develop the necessary skills, it was the application of knowledge and continued practice during tele-consults (i.e. the service exchanges) which increased member's satisfaction in providing care. One of the nurses at NH1 reported that

"If we check the heart, it was very difficult to differentiate whether it is normal or abnormal. When we do the Tele G, we have a doctor beside us to guide us. We ask the doctor to check if we are doing it right... When next time we encounter the case, we do even better. So it is a way of learning and improving ourselves"

Taken together, the findings from $\mathrm{H} 1 \mathrm{~b}$ and $\mathrm{H} 2 \mathrm{~b}$ suggest that while resource exchanges were important for members to uplift their nurses' skills, it was the application of the competencies which resulted in improved satisfaction. As members became involved in service exchanges, it improved their nurse's satisfaction as they were able to handle their residents over technology, avoiding hassles faced during physical visits. 


\section{Cost effectiveness}

In hypothesis $1(\mathrm{c})$ and 2(c) we argue that more resource exchanges and service exchanges will be associated with cost effectiveness for the members. Consistent with our hypothesis H1(c) we found that more service exchanges resulted in cost effectiveness for members. Prior to telegeriatrics, all patients had to be rushed to acute hospitals for consultations resulting in increased reliability on acute hospitals. This was costly to members as cost of accessing specialists, cost of transportation and unnecessary costs of admission in acute hospitals increased cost to members. In addition, a nurse had to accompany the patients which further increased cost to the members as these resources were in shortage and worked for fixed hours. As nursing homes started engaging in more tele-consultations, they were able to provide timely care to patients in-house whereby some unnecessary admissions and outpatient visits were avoided which resulted in cost savings for the nursing homes.

In hypothesis $\mathrm{H} 2(\mathrm{c})$ we argued that with more resource exchanges, nurses would become more trained in diagnosing patient conditions and unnecessary visits to hospitals would be avoided which in turn would improve cost effectiveness. However, we did not find support for this hypothesis suggesting that more trained resources would not necessarily result in cost effectiveness. Cost effectiveness was achieved only through service exchanges when patients were treated by the specialists.

Overall, the pattern of findings across service and resource exchanges and the three aspects of value to members suggests that while participation in resource exchanges uplifts nurse's skillset resulting in improved clinical outcomes, further participation in service exchanges with the keystone organization brings in additional value to members measured in form of user satisfaction and cost effectiveness. 


\section{Impact of Relational Exchanges on Service and Resource Exchanges}

In hypothesis $\mathrm{H} 3$ and $\mathrm{H} 4$, we argued that more relational exchanges will be associated with more service and resource exchanges among the members and the keystone organization over time. Consistent with our hypothesis H4, we found that as nursing homes started participating in relational exchanges, it increased their willingness to engage in resource exchanges. Nursing homes, on average have nearly 200 residents who are managed by a relatively small pool of 15-17 nurses and about 40 nursing aides. These nurses are however not clinically proficient to manage patients' medical conditions. The tele-geriatrics ecosystem required the nursing homes to attend nine-month long clinical training program and undergo various assessments in order to become qualified for conducting tele-consultations. However, since nursing staff were critical resources for nursing homes, training required that the nursing homes' commit their nurses to undergo training beyond their normal work hours. By engaging in relational exchanges with Acute North, nursing homes were able to voice their concerns on various issues such as the timing of the training sessions, or difficulties with understanding the training content. Acute North in turn responded to nursing homes' concerns in these meetings by making adjustments to training content and working around the nursing homes' work schedules, to fit in the training sessions. Relational exchanges hence increased willingness of nursing homes to pool their critical resources for tele-geriatrics as their concerns were being appropriately addressed. Infact, our results suggest that while clinical outcomes are improved through participation in resource exchanges by $\beta_{2}=-2.7789$, participation in relational exchanges $\left(\gamma_{1}=\right.$ 0.013622 ) can further enhance the clinical outcomes by a magnitude of -2.680 .

In Hypothesis H3, we argued that as service exchanges required coordination among members and the keystone organization, more relational exchanges will result in more service 
exchanges as members will be able to define roles, responsibilities and protocols for exchange (such as what information needs to be shared prior to the start of consultation or after the consultation) with the keystone organization. Relational exchanges will also provide a platform for members to resolve issues faced during service exchange. For example, it was reported that while nursing homes had been trained to examine a patient in a certain standardized manner, doctors sometimes insisted on their own approach, which in turn led to interruptions during consultation. By engaging in relational exchanges, nursing homes could resolve such issues which would increase their willingness to engage in service exchanges. However, we did not find support for this hypothesis.

On further analysis of the data, we found that service exchanges were also affected by the turnover in staff faced by nursing homes that undermined the mutual understanding built from prior relational exchanges. For example, we found that $\mathrm{NH} 2$ had been facing tremendous turnover which resulted in loss of several trained nurses. In addition, $\mathrm{NH} 2$ also underwent changes in management resulting in loss of continuity built previously in relationships. Turnover was a major concern for all nursing homes as it resulted in loss of continuity. This impacted service exchanges as these were more sophisticated in nature and required experienced nursing staff to conduct the tele-consultation session.

The overall pattern of findings revealed from our data analysis suggests important considerations for members in service ecosystems. First, in service ecosystems, the early assertion is that joining the ecosystem will result in benefits for the members. Our findings however suggest that it is the engagement in value co-creational exchanges- service, resource and relational, that in fact results in value to members. Second, our results further demonstrate that through engagement in value co-creational exchanges, members can derive value both in 
economic (cost effectiveness) and non-economic (quality of service measured as clinical outcomes and user satisfaction) forms. Third, our results show that while resource exchanges are necessary, they are not sufficient to generate all forms of value to members. In a service ecosystem, members are further required to engage in service exchange in order to meet customer needs. This contrasts from a goods-dominant ecosystem, where focus is on the tangible goods that are exchanged among the members. As service exchanges are more sophisticated requiring application of competencies, it is only when members engage in service exchanges, they derive wider forms of value.

\section{Theoretical Contribution}

This study contributes to the literature of value co-creation in service ecosystems in three ways. First, by taking the service-dominant view, we specifically define the three value cocreational exchanges (service exchanges, resource exchanges and relational exchanges) that occur among members and the keystone in a service ecosystem. As organizations today are increasingly seeking partnerships to co-create value which they cannot develop individually on their own, this joint co-creation of value holds significant importance. However, there has been less conceptual as well as empirical work on the very joint nature of value co-creation (Goran 2012; Lusch and Nambisan 2015; Sarker et al. 2012). Our study provides a theoretical conceptualization of this value co-creation and then examines how value to members is affected by their participation in these exchanges. In doing this, the study enriches the literature by revealing several important findings for value co-creation in service ecosystems.

Second, this study evaluates different forms of value to members - economic (cost effectiveness) and non-economic value (quality of service). Prior studies that have examined value to members have not considered the value to members when they engage in value co- 
creational exchanges and have mostly examined market value of members (Ceccagnoli et al. 2012; Han et al. 2012). However, this alone may result in an under-estimation of value to members. It is only by capturing the process of value co-creational exchanges, that the true value to members can be assessed. By assessing value that members derive by participation in value co-creational exchanges, this study provides a richer view of value that members derive from a service ecosystem.

Third, our proposed model on value to members in a service ecosystem provides a novel view of relationships between the three value co-creational exchanges. Our findings reveal that while service and resource exchanges directly affect the value to members in an ecosystem, relational exchanges are important to encourage members to engage in more resource and service exchanges. Although in our case, service exchanges were significantly impacted by the turnover in staffing, we believe theoretically, service exchanges would benefit from participation in relational exchanges. Further research on different value co-creational exchanges can validate this theoretical propositions.

Finally, our research context of telemedicine enabled integrated care ecosystem contributes to the growing literature on integrated care collaborations. Prior research in integrated care has sufficiently highlighted the different forms of value to members in an ecosystem. It has also examined how integrated care interventions have impacted these various forms of value (Balasubramanian and Spurgeon 2012; Becevic et al. 2015; Rojas and Gagnon 2008; Whittaker and Wade 2014). However, very few studies (Beech et al. 2013; Ruesch et al. 2012; Sandoval Garrido et al. 2014) have now started to recognize a handful of factors (number of nurses, integrated governance) which may influence the value co-creation in such collaborations. This study provides a comprehensive framework for organizations to understand 
the different value co-creational exchanges in a service ecosystem and how these impact the value to members. Specifically, our context provides evidence of what exchanges impact what form of value to members in an integrated care ecosystem.

\section{Practical Implications}

Our results also have several implications for both the members and the keystone organization. First, with a growing shift towards a consumer centric service economy, value is now co-created through reciprocal exchanges among the organizations. Hence value co-created is idiosyncratic to meet customer needs and is seen emerging and unfolding over time through these exchanges. This is a significant change from a goods-based perspective where actors were independent and focused on exchange of tangible goods whose value was determined by the price customer was willing to pay (Vargo and Lusch 2008). This change has shifted the focus towards mutual and reciprocal value co-creational exchanges among the keystone organization and the members.

This study provides a detailed framework for the keystone organization to decipher how it can co-create value together with members. For example, a keystone organization may invest in relational exchanges to create transparency on sharing of critical resources in the ecosystem. The keystone organization may hence be able to maintain a sustained value proposition for its members.

Second, by explicating three exchanges that occur between members and the keystone organization, our study provides a framework for members to assess how their participation effects the different forms of value they draw from an ecosystem. Since value co-creation requires collaborative exchanges with the keystone organization, members can closely assess the impact of their exchanges over time. For example, by participating in resource exchanges, 
members' resources may become more co-specialized and improved in form whereas participation in service exchanges may improve cost effectiveness for members. As exchanges may impact different forms of value, it provides a clearer view to members on how their actions impact the value they draw from an ecosystem.

Last, our results also have implications for integrated care ecosystems which have been facing challenges of sustainability over time (Bevan and Janus 2011; Tsasis et al. 2012). This mainly arises from diversity of providers and lack of trusting relationships among them (Bevan and Janus 2011; Cebul et al. 2008; Leichsenring 2004; Ling et al. 2012b; Montenegro et al. 2011; Pate et al. 2010; Pike and Mongan 2014; Pomerantz et al. 2009). Our study reveals three value co-creational exchanges that the integrated care ecosystems may focus on to deliver healthcare services.

\section{Conclusion and Future Research}

This study is motivated to empirically examine the value to members in a service ecosystem. For this, we first propose a model by explicating three value co-creational exchanges among members and the keystone organization. We then empirically test our model on a longitudinal dataset in the context of a telemedicine enabled integrated care ecosystem. Our findings reveal that members accrue value (both economic and non- economic) by participating in these value co-creational exchanges. Our results have implications for both members and the keystone organization.

Future research can extend our findings and address some limitations of our study. First, this study takes a longitudinal dataset to examine value co-creation which comprises of a keystone organization and four members. While this presents an opportunity to scrutinize value co-creational exchanges closely, it also raises concerns of generalizability as we are restricted by 
our context and number of members. Further studies can build on our findings and examine our model in larger ecosystems which comprise of thousands of members. These contexts may unravel new ways by which organizations engage in value co-creational exchanges.

Second, we examine our model in an integrated care context where value to members (economic and non-economic) is usually explained through clinical outcomes, cost effectiveness and user satisfaction. However, contexts such as service innovation or ERP may provide members value in terms of increased access to customers or development of innovative solutions. Future studies could examine these different forms of value and how they are impacted by value co-creational exchanges.

Third, in this study, we focus on dyadic value co-creational exchanges that occur between the keystone organization and members. However, members in an ecosystem may also co-create value without the involvement of the keystone organization or they may exist other forms of organizational exchanges (involving more than two members) (Lusch and Nambisan 2015). Future studies could examine how value co-creational exchanges occur in such cases and how do members accrue benefits from the same. 


\section{CHAPTER 4.}

\section{SUMMARY AND IMPLICATIONS OF FINDINGS}

This chapter first provides an integrated summary of the findings from the previous two studies. Then it discusses the contribution to theory and implications for practice. This is followed by suggestions for future research.

\section{SUMMARY OF FINDINGS}

This thesis takes a mixed methods approach to investigate two critical aspects of business ecosystems which affect its sustainability - first the tensions during evolution and second the cocreation of value and the benefits accrued by members from the ecosystems. These two aspects hold significant importance for both the keystone organization and the members. While it is crucial for the keystone organization to be vigilant in managing tensions, members also need to understand how their participation in ecosystems effects the value they derive from the ecosystem. For this, two separate but inter-related studies are conducted in the context of healthcare which has recently witnessed increase in ecosystems to manage care. Together, findings from both the studies highlight critical roles played by both the keystone organization and members in sustainable evolution of the ecosystem.

The first essay takes a qualitative case study approach to address the research question of

how the keystone organization manages the evolution of an ecosystem by addressing the emerging tensions. The findings and analysis reveal three broad phases of evolution- member enrollment, joint capabilities development and ecosystem renewal. Each of this phase is characterized by a dominant tension: individual vs collective identifications, actual vs expected capabilities and existing vs new commitments which significantly influences the evolution of the 
ecosystem. Further, the findings also reveal the specific orchestration actions, namely, Identity Adaptation, Capability Adaptation and Boundary Adaptation which are taken in response to specifically manage these tensions.

The second essay takes a quantitative approach of panel data analysis with fixed effects to address the research question of how value cocreation exchanges among the members and the keystone organization influence the value to members over time. Drawing on service view of ecosystems and broader value co-creation literature, the study first explicates three value cocreation exchanges- service exchange, resource exchange and relational exchange and then measures their effect on both economic and non-economic value to members. The findings reveal that all three value co-creational exchanges are vital to the value derived by members, albeit differently. While service exchanges improve the economic value (measured as cost effectiveness) derived by members, non-economic value such as clinical outcomes is improved by participation in resource exchanges. Also, relational exchanges were found to increase the willingness of members to engage in more resource exchanges.

Together, this mixed methods investigation and perspectives from both the keystone organization and the members provides a detailed assessment for ecosystem sustainability. First, ecosystems face challenges arising from within and environmental context which often manifest as tensions or competing demands in the ecosystem. The findings highlight these tensions (along with their sources and effects) and the specific adaptive orchestration actions taken by the keystone organization to manage these tensions. Second, mere participation in ecosystems is not sufficient to generate wider range of benefits to members as value is co-created through mutual and reciprocal exchanges among the members and the keystone organization over time. The findings specifically reveal the relationships between the value co-creational exchanges on 
different types of value to members. Interestingly, these findings provide important considerations for both theory and practice.

\section{CONTRIBUTIONS TO THEORY}

This thesis holds several important contributions for the literature in ecosystems, orchestration and value co-creation.

This thesis contributes to the ecosystem literature in the following ways. First, this thesis develops a comprehensive process model of ecosystem evolution. While prior research has suggested that ecosystems evolve and this evolution is subjected to challenges or tensions arising from internal and external environment (Garud et al. 2002; Lindgren et al. 2015; Wareham et al. 2014). However, these two aspects have been studied separately. The process model developed in Essay 1 reconciles these two views to identify three salient tensions in each phase, namely, individual vs collective identifications (member enrollment phase), actual vs expected capabilities (joint capabilities development phase) and new vs existing commitments (ecosystem renewal phase).

In doing so, this thesis first extends the concept of tensions in organizational literature in a single organization to more complex ecosystem analysis. We identify two specific tensions of actual vs expected capabilities and existing vs new commitments which are more relevant in the context of ecosystems. This also adds to the recent stream of research in ecosystems that has started to examine the tensions that impact the ecosystem evolution (Garud et al. 2002; Lindgren et al. 2015; Wareham et al. 2014).

Second, this thesis, extends the literature on ecosystem orchestration (Dhanaraj and Parkhe 2006; Nambisan and Sawhney 2011) which has predominantly focused on the routine actions taken by the keystone organization to evolve the ecosystem. The findings from Essay 1 
reveal that along with the necessary routine actions, the keystone organization enacts adaptive orchestration actions which address the tensions that arise in each phase of evolution. While routine orchestration actions are standard necessary to steer the ecosystem evolution, adaptive orchestration actions are responsive to the tensions and emergent in nature as these incorporate feedback and member concerns.

Finally, this thesis contributes to the recent stream of work in ecosystems which evaluates the benefits to members (Ceccagnoli et al. 2012; Han et al. 2012). While studies have clearly indicated that exchanges among the members and the keystone organization are critical components of value co-creation, yet the participation of members has been considered a onetime event. Our conceptualization of value co-creational exchanges- namely, service exchanges, resource exchanges and relational exchanges in Essay 2 provide clarity on different exchanges in ecosystem. Further, the thesis examines the relationship of value co-creation exchanges with both economic and non-economic benefits to members over time. The results show that participation of members in ecosystems is not a one-time event, rather ongoing and coordinated with the keystone organization.

\section{IMPLICATIONS FOR PRACTICE}

The findings from this thesis have several practical implications. We first discuss implications for both the keystone organization and the members in ecosystems and then discuss implications for integrated care.

\section{Implications for the Keystone Organization and the Members}

As ecosystems are characterized by diverse members spanning across industries, the keystone organization needs to be vigilant and responsive to the member needs and changes in environmental context. The findings sensitize the keystone organization to the specific tensions 
which are likely to be salient in each phase of evolution and how these can be addressed with adaptive orchestration actions. For this, the keystone organization could engage and seek feedback and continue to invest in creation of value for its members by engaging in reciprocal and mutual exchanges.

Second, with a shift towards consumer centric service based economy, this thesis takes this recent service view of ecosystems which suggests that value is co-created through reciprocal exchanges among organizations. With this, we explicate three different value co-creational exchanges and provide a detailed framework which the keystone organization can use to decipher how it can co-create value with its members. For example, the keystone organization may engage in more relational exchanges to establish mechanisms and create transparency on resource shared with the members. With such transparency in place, the keystone organization can improve the commitment of members to its ecosystem.

Third, these findings also have implications for the members as it provides clarity to assess how their participation in the ecosystem effects the different forms of value they draw from the ecosystem. For example, with participation in resource exchanges, the members' resources may become more co-specialized and participation in relational exchanges can help them develop standards on the use of these co-specialized resources.

\section{Implications for Integrated Care}

The findings of this thesis also have implications for integrated care ecosystems which have been facing challenges of sustainability over time. First, while integrated care has been the most frequently recommended solution to achieve consistent and affordable care, it is still impeded with the challenges arising from different organizational identities and lack of trusting relationships. Our findings suggest that some of these challenges can be addressed by 
implementing adaptive orchestration actions such as developing governance mechanisms to build transparency or developing a collective identity by leveraging existing relationships. The findings also reveal that the more effective governance approach taken by the keystone organization has to be one that instills equal partnership rather than one focused on top down decision making.

Second, prior research that has assessed telemedicine enabled integrated care outcomes has been restricted to a design period of six months or different patient samples where patient related outcomes are measured before and after telemedicine intervention to gauge the benefits (Balasubramanian and Spurgeon 2012; Becevic et al. 2015; Rojas and Gagnon 2008; Whittaker and Wade 2014). Our findings on other hand reveal that complex exchanges occur in an ecosystem and which in turn influence the co-creation of value. For example, our data shows that non- economic outcomes such as clinical outcomes were improved by resource exchanges rather than the service exchange itself. At the same time, transparency in processes and decision making motivated the members further to invest their critical healthcare resources in the ecosystem. Such findings call for more holistic assessment of integrated care outcomes.

Finally, the findings from this thesis may not be limited to telemedicine enabled integrated care, but also be applicable to other settings such as electronic health records or home healthcare which requires coordination among diverse stakeholders. As development and sustainability of healthcare ecosystems is extremely challenging, our findings offer a comprehensive framework to organizations to unpack both the complexities in evolution as well as value co-creational exchanges among members. 


\section{DIRECTIONS FOR FUTURE RESEARCH}

While we have made every attempt to conduct comprehensive studies to address the gaps in the literature, nonetheless our results and analysis are suggestive of further important avenues for future research.

First, while the context of healthcare offers novelty to examine ecosystems as it is characterized by resource scarce environments and public private partnerships, future research is required to strengthen the generalizability of our models. For example, ecosystems in high technology industries such as those of Amazon or eBay often boast of thousands of partners. This may not only increase the complexity of relationships, but also challenge the ability of the keystone organization to manage resources and processes in such vast ecosystems. Infact, it requires the keystone organization to enact additional adaptive orchestration actions to manage these growing complexities. Future research can build on the findings of Essay 1 to identify such adaptive orchestration actions.

Second, while much work has been done in the past on the role of the keystone organization and orchestration actions in the evolution, less is known about the interactions among the members and how it effects the evolution. For example, it has been noted in the prior literature that members leverage the common platforms to foster interactions with other members and to engage in problem solving, knowledge sharing and learning (Jha et al 2016). The findings from Essay 1 in last phase also point out the different governance platforms established by the keystone organization which were in turn used by members to share their joint training needs. Future research can further build on this to focus on the role of members in ecosystem evolution.

Third, in this thesis, one crucial finding of Essay 1 pertains to tensions where I identify three tensions along with their sources and effects that are salient in each phase of the ecosystem 
driving its evolution. Very few studies (Garud et al. 2002; Lindgren et al. 2015; Wareham et al. 2014) in ecosystems have currently highlighted the notion of tensions in ecosystems, however these have not observed how tensions evolve over time. This also holds implications for the broader literature in organization theory where seminal papers have conceptualized tensions within a single organization (Lewis \& Smith, 2011). Future research can build and expand on these studies to examine the tensions in larger ecosystems or other organizational forms.

Fourth, currently the examination of value co-creation in Essay 2 comprises of a longitudinal dataset collected from four members and a keystone organization. While in itself, the data offers immense potential to operationalize critical value co-creational exchanges through both qualitative and quantitative measures, future research could examine the proposed models in larger ecosystems. This could potentially unleash more ways of operationalization of key antecedents and build more understanding on how organizations engage in value co-creational exchanges. This also highlights another related avenue for further research. The thesis in the integrated care context examines the value to members through clinical outcomes, cost effectiveness and user satisfaction. Future studies should examine different forms of value especially arising from contexts such as service innovation or ERP where value to members exists is in form of increased access to customers or development of innovative solutions.

Finally, in Essay 2, we focus on the dyadic value co-creational exchanges that occur between the keystone organization and members. However, members in an ecosystem may also co-create value without the involvement of the keystone organization or they may exist other forms of organizational exchanges (involving more than two members) (Lusch and Nambisan 2015). Future studies could examine how value co-creational exchanges occur in such cases and how do members accrue benefits from the same. 


\section{REFERENCES}

Adner, R. 2006. "Match Your Innovation Strategy to Your Innovation Ecosystem," Harvard business review (84:4), p. 98.

Adner, R., and Kapoor, R. 2010. "Value Creation in Innovation Ecosystems: How the Structure of Technological Interdependence Affects Firm Performance in New Technology Generations," Strategic management journal (31:3), pp. 306-333.

Balasubramanian, S., and Spurgeon, P. 2012. "Integrated Care for Chronic Pain Management in the National Health Service: Barriers and Possibilities for Service Development-a Survey," International Journal of Clinical Leadership (17:4).

Banoun, A., Dufour, L., and Andiappan, M. 2016. "Evolution of a Service Ecosystem: Longitudinal Evidence from Multiple Shared Services Centers Based on the Economies of Worth Framework," Journal of Business Research (69:8), pp. 2990-2998.

Barrett, M., Davidson, E., Prabhu, J., and Vargo, S. L. 2015. "Service Innovation in the Digital Age: Key Contributions and Future Directions," MIS quarterly (39:1), pp. 135-154.

Batterink, M. H., Wubben, E. F., Klerkx, L., and Omta, S. 2010. "Orchestrating Innovation Networks: The Case of Innovation Brokers in the Agri-Food Sector," Entrepreneurship and regional development (22:1), pp. 47-76.

Becevic, M., Boren, S., Mutrux, R., Shah, Z., and Banerjee, S. 2015. "User Satisfaction with Telehealth: Study of Patients, Providers, and Coordinators," The health care manager (34:4), pp. 337-349.

Beech, R., Henderson, C., Ashby, S., Dickinson, A., Sheaff, R., Windle, K., Wistow, G., and Knapp, M. 2013. "Does Integrated Governance Lead to Integrated Patient Care? Findings from the Innovation Forum," Health \& social care in the community (21:6), pp. 598-605.

Bevan, G., and Janus, K. 2011. "Why Hasn't Integrated Health Care Developed Widely in the United States and Not at All in England?," Journal of Health Politics, Policy and Law (36:1), pp. 141-164.

Beyer, M., Kuhn, K. A., Meiler, C., Jablonski, S., and Lenz, R. 2004. "Towards a Flexible, Process-Oriented It Architecture for an Integrated Healthcare Network," Proceedings of the 2004 ACM symposium on Applied computing: ACM, pp. 264-271.

Busse, R., and Stahl, J. 2014. "Integrated Care Experiences and Outcomes in Germany, the Netherlands, and England," Health Affairs (33:9), pp. 1549-1558.

Cebul, R. D., Rebitzer, J. B., Taylor, L. J., and Votruba, M. 2008. "Organizational Fragmentation and Care Quality in the Us Health Care System," National Bureau of Economic Research.

Ceccagnoli, M., Forman, C., Huang, P., and Wu, D. 2012. "Cocreation of Value in a Platform Ecosystem: The Case of Enterprise Software," MIS Quarterly (36:1), p. 263.

Chae, Y. M., Lee, J. H., Ho, S. H., Kim, H. J., Jun, K. H., and Won, J. U. 2001. "Patient Satisfaction with Telemedicine in Home Health Services for the Elderly," International journal of medical informatics (61:2), pp. 167-173.

Curry, N., Harris, M., Gunn, L., Pappas, Y., Blunt, I., Soljak, M., Mastellos, N., Holder, H., Smith, J., and Majeed, A. 2013. "Integrated Care Pilot in North West London: A Mixed Methods Evaluation," International Journal of Integrated Care (13:3).

Cusumano, M. A., and Gawer, A. 2002. "The Elements of Platform Leadership," MIT Sloan management review (43:3), p. 51. 
Darkins, A., Ryan, P., Kobb, R., Foster, L., Edmonson, E., and Wakefield, B. 2008. "The Systematic Implementation of Health Informatics, Home Telehealth and Disease Management to Support the Care of Veteran Patients with Chronic Conditions," Telemedicine and e-Health).

de Stampa, M., Vedel, I., Buyck, J.-F., Lapointe, L., Bergman, H., Beland, F., and Ankri, J. 2014. "Impact on Hospital Admissions of an Integrated Primary Care Model for Very Frail Elderly Patients," Archives of gerontology and geriatrics (58:3), pp. 350-355.

Dhanaraj, C., and Parkhe, A. 2006. "Orchestrating Innovation Networks," Academy of management review (31:3), pp. 659-669.

Dubé, L., and Paré, G. 2003. "Rigor in Information Systems Positivist Case Research: Current Practices, Trends, and Recommendations," MIS quarterly), pp. 597-636.

Dyer, J. H., and Hatch, N. W. 2006. "Relation-Specific Capabilities and Barriers to Knowledge Transfers: Creating Advantage through Network Relationships," Strategic management journal (27:8), pp. 701-719.

Dyer, J. H., and Nobeoka, K. 2000. "Creating and Managing a High-Performance KnowledgeSharing Network: The Toyota Case," Strategic management journal (21:3), pp. 345-367.

Dyer, J. H., and Singh, H. 1998. "The Relational View: Cooperative Strategy and Sources of Interorganizational Competitive Advantage," Academy of management review (23:4), pp. 660-679.

Eisenhardt, K. M. 1989. "Building Theories from Case Study Research," Academy of management review (14:4), pp. 532-550.

Eisenmann, T., Parker, G., and Van Alstyne, M. W. 2006. "Strategies for Two-Sided Markets," Harvard business review (84:10), p. 92.

Finkelstein, S. M., Speedie, S. M., and Potthoff, S. 2006. "Home Telehealth Improves Clinical Outcomes at Lower Cost for Home Healthcare," Telemedicine Journal \& e-Health (12:2), pp. 128-136.

Franzini, L., Sail, K. R., Thomas, E. J., and Wueste, L. 2011. "Costs and Cost-Effectiveness of a Telemedicine Intensive Care Unit Program in 6 Intensive Care Units in a Large Health Care System," Journal of critical care (26:3), pp. 329. e321-329. e326.

Garud, R., Jain, S., and Kumaraswamy, A. 2002. "Institutional Entrepreneurship in the Sponsorship of Common Technological Standards: The Case of Sun Microsystems and Java," Academy of management journal (45:1), pp. 196-214.

Gausdal, A. H., and Nilsen, E. R. 2011. "Orchestrating Innovative Sme Networks. The Case of "Healthinnovation"," Journal of the Knowledge Economy (2:4), pp. 586-600.

Gnyawali, D. R., and Madhavan, R. 2001. "Cooperative Networks and Competitive Dynamics: A Structural Embeddedness Perspective," Academy of Management review (26:3), pp. 431445.

Goran, S. F. 2012. "Measuring Tele-Icu Impact: Does It Optimize Quality Outcomes for the Critically Ill Patient?," Journal of nursing management (20:3), pp. 414-428.

Greaves, F., Pappas, Y., Bardsley, M., Harris, M., Curry, N., Holder, H., Blunt, I., Soljak, M., Gunn, L., and Majeed, A. 2013. "Evaluation of Complex Integrated Care Programmes: The Approach in North West London," International Journal of Integrated Care (13:1).

Grover, V., and Kohli, R. 2012. "Cocreating It Value: New Capabilities and Metrics for Multifirm Environments," MIS Quarterly (36:1), pp. 225-232.

Hailey, D. M., and Crowe, B. L. 2000. "Assessing the Economic Impact of Telemedicine," Disease Management and Health Outcomes (7:4), pp. 187-192. 
Han, K., Oh, W., Im, K. S., Chang, R. M., Oh, H., and Pinsonneault, A. 2012. "Value Cocreation and Wealth Spillover in Open Innovation Alliances," MIS Quarterly (36:1), pp. 291-325.

Iansiti, M., and Levien, R. 2004. "Strategy as Ecology," Harvard business review (82:3), pp. 6881.

Ignatowicz, A., Greenfield, G., Pappas, Y., Car, J., Majeed, A., and Harris, M. 2014. "Achieving Provider Engagement Providers' Perceptions of Implementing and Delivering Integrated Care," Qualitative Health Research (24:12), pp. 1711-1720.

Jacobs, K., Blanchard, B., and Baker, N. 2012. "Telehealth and Ergonomics: A Pilot Study," Technology and Health Care (20:5), pp. 445-458.

Jha, S. K., Pinsonneault, A., and Dubé, L. 2016. "The Evolution of an Ict Platform-Enabled Ecosystem for Poverty Alleviation: The Case of Ekutir," MIS Quarterly: Management Information Systems (40:2), pp. 431-445.

Kandiah, G., and Gossain, S. 1998. "Reinventing Value: The New Business Ecosystem," Strategy \& Leadership (26:5), pp. 28-33.

Kim, H., Lee, J.-N., and Han, J. 2010. "The Role of It in Business Ecosystems," Communications of the $A C M(53: 5)$, pp. 151-156.

Knight, P., Bonney, A., Teuss, G., Guppy, M., Lafferre, D., Mullan, J., and Barnett, S. 2016. "Positive Clinical Outcomes Are Synergistic with Positive Educational Outcomes When Using Telehealth Consulting in General Practice: A Mixed-Methods Study," Journal of medical Internet research (18:2).

Kodner, D. L., and Spreeuwenberg, C. 2002. "Integrated Care: Meaning, Logic, Applications, and Implications-a Discussion Paper," International journal of integrated care (2:4).

Larcher, B., Arisi, E., Berloffa, F., Demichelis, F., Eccher, C., Galligioni, E., Galvagni, M., Martini, G., Sboner, A., and Tomio, L. 2003. "Analysis of User-Satisfaction with the Use of a Teleconsultation System in Oncology," Medical informatics and the Internet in medicine (28:2), pp. 73-84.

Leichsenring, K. 2004. "Developing Integrated Health and Social Care Services for Older Persons in Europe," International journal of integrated care (4:3).

Leong, C. M. L., Pan, S. L., Newell, S., and Cui, L. 2016. "The Emergence of Self-Organizing E-Commerce Ecosystems in Remote Villages of China: A Tale of Digital Empowerment for Rural Development," Mis Quarterly (40:2), pp. 475-484.

Lewis, M. W. 2000. "Exploring Paradox: Toward a More Comprehensive Guide," Academy of Management review (25:4), pp. 760-776.

Lindgren, R., Eriksson, O., and Lyytinen, K. 2015. "Managing Identity Tensions During Mobile Ecosystem Evolution," Journal of Information Technology (30:3), pp. 229-244.

Ling, T., Brereton, L., Conklin, A., Newbould, J., and Roland, M. 2012a. "Barriers and Facilitators to Integrating Care: Experiences from the English Integrated Care Pilots," International journal of integrated care (12:129), pp. 1-12.

Ling, T., Brereton, L., Conklin, A., Newbould, J., and Roland, M. 2012b. "Barriers and Facilitators to Integrating Care: Experiences from the English Integrated Care Pilots," International journal of integrated care (12:5).

Lusch, R. F., and Nambisan, S. 2015. "Service Innovation: A Service-Dominant Logic Perspective," Mis Quarterly (39:1), pp. 155-175.

Mayhew, L. 2009. "On the Effectiveness of Care Co-Ordination Services Aimed at Preventing Hospital Admissions and Emergency Attendances," Health care management science (12:3), pp. 269-284. 
McLean, S., Nurmatov, U., Liu, J., Pagliari, C., Car, J., and Sheikh, A. 2011. "Telehealthcare for Chronic Obstructive Pulmonary Disease," Cochrane Database Syst Rev (7:7).

Ménard, C. 1995. "Markets as Institutions Versus Organizations as Markets? Disentangling Some Fundamental Concepts," Journal of economic behavior \& organization (28:2), pp. 161-182.

Merz, M. Y., Czerwinski, D., and Merz, M. A. 2013. "Exploring the Antecedents for Value Cocreation During Healthcare Service Provision," Journal of Business and Behavior Sciences (25:2), p. 152.

Minkman, M. M. 2012. "The Current State of Integrated Care: An Overview," Journal of Integrated Care (20:6), pp. 346-358.

Montenegro, H., Holder, R., Ramagem, C., Urrutia, S., Fabrega, R., Tasca, R., Alfaro, G., Salgado, O., and Angelica Gomes, M. 2011. "Combating Health Care Fragmentation through Integrated Health Service Delivery Networks in the Americas: Lessons Learned," Journal of Integrated Care (19:5), pp. 5-16.

Moore, J. F. 1993. "Predators and Prey: A New Ecology of Competition," Harvard business review (71:3), pp. 75-83.

Morris, M., and Yoritomo, W. 2015. "Global Health Care Outlook: Common Goals, Competing Priorities." Retrieved from deloitte. com: http://www2/. deloitte. com/au/en/pages/lifesciences-and-healthcare/articles/global-health-care-sector-outlook. html.

Myers, R. 1990. "Detecting and Combating Multicollinearity," Classical and Modern Regression), pp. 384-385.

Nambisan, S., and Sawhney, M. 2011. "Orchestration Processes in Network-Centric Innovation: Evidence from the Field," The Academy of Management Perspectives (25:3), pp. 40-57.

Neghina, C., Caniëls, M. C., Bloemer, J. M., and van Birgelen, M. J. 2014. "Value Cocreation in Service Interactions Dimensions and Antecedents," Marketing Theory), p. 1470593114552580.

Nordheim, L. V., Haavind, M. T., and Iversen, M. M. 2014. "Effect of Telemedicine Follow-up Care of Leg and Foot Ulcers: A Systematic Review," BMC health services research $(14: 1)$, p. 1.

Norman, G. 2011. "The Solution to Ongoing Health Care Challenges: Creating Integrated Personal Health Ecosystems," Population health management (14:2), pp. 57-58.

Pate, J., Fischbacher, M., and Mackinnon, J. 2010. "Health Improvement: Countervailing Pillars of Partnership and Profession," Journal of health organization and management (24:2), pp. 200-217.

Paul, D. L. 2006. "Collaborative Activities in Virtual Settings: A Knowledge Management Perspective of Telemedicine," Journal of Management Information Systems (22:4), pp. 143-176.

Peltoniemi, M. 2006. "Preliminary Theoretical Framework for the Study of Business Ecosystems," Emergence: Complexity \& Organization (8:1).

Pike, B., and Mongan, D. 2014. "The Integration of Health and Social Care Services," Health Research Board, Dublin).

Podolny, J. M., and Page, K. L. 1998. "Network Forms of Organization," Annual review of sociology), pp. 57-76.

Pomerantz, A. S., Corson, J. A., and Detzer, M. J. 2009. "The Challenge of Integrated Care for Mental Health: Leaving the 50 Minute Hour and Other Sacred Things," Journal of Clinical Psychology in Medical Settings (16:1), pp. 40-46. 
Prince, K., Barrett, M., and Oborn, E. 2014. "Dialogical Strategies for Orchestrating Strategic Innovation Networks: The Case of the Internet of Things," Information and Organization (24:2), pp. 106-127.

Rai, A., Pavlou, P. A., Im, G., and Du, S. 2012. "Interfirm It Capability Profiles and Communications for Cocreating Relational Value: Evidence from the Logistics Industry," MIS Quarterly (36:1), pp. 233-262.

Rai, A., and Sambamurthy, V. 2006. "Editorial Notes-the Growth of Interest in Services Management: Opportunities for Information Systems Scholars," Information Systems Research (17:4), pp. 327-331.

Rajan, B., Seidmann, A., and Dorsey, E. R. 2013. "The Competitive Business Impact of Using Telemedicine for the Treatment of Patients with Chronic Conditions," Journal of Management Information Systems (30:2), pp. 127-158.

Reeves, M., Levin, S., and Ueda, D. 2016. "The Big Idea the Biology of Corporate Survival," HARVARD BUSINESS REVIEW (94:1-2), pp. 46-55.

Robertson, P. J., and Choi, T. 2010. "Ecological Governance: Organizing Principles for an Emerging Era," Public administration review (70:s1), pp. s89-s99.

Roine, R., Ohinmaa, A., and Hailey, D. 2001. "Assessing Telemedicine: A Systematic Review of the Literature," Canadian Medical Association Journal (165:6), pp. 765-771.

Rojas, S. V., and Gagnon, M.-P. 2008. "A Systematic Review of the Key Indicators for Assessing Telehomecare Cost-Effectiveness," Telemedicine and e-Health (14:9), pp. 896904.

Ruesch, C., Mossakowski, J., Forrest, J., Hayes, M., Jahrsdoerfer, M., Comeau, E., and Singleton, M. 2012. "Using Nursing Expertise and Telemedicine to Increase Nursing Collaboration and Improve Patient Outcomes," TELEMEDICINE and e-HEALTH (18:8), pp. 591-595.

Sandoval Garrido, F. A., Tamiya, N., Kashiwagi, M., Miyata, S., Okochi, J., Moriyama, Y., Yamaoka, Y., and Takamuku, K. 2014. "Relationship between Structural Characteristics and Outcome Quality Indicators at Health Care Facilities for the Elderly Requiring LongTerm Care in Japan from a Nationwide Survey," Geriatrics \& gerontology international (14:2), pp. 301-308.

Sarker, S., Sarker, S., Sahaym, A., and Bjørn-Andersen, N. 2012. "Exploring Value Cocreation in Relationships between an Erp Vendor and Its Partners: A Revelatory Case Study," MIS quarterly (36:1), pp. 317-338.

Schwamm, L. H. 2014. "Telehealth: Seven Strategies to Successfully Implement Disruptive Technology and Transform Health Care," Health Affairs (33:2), pp. 200-206.

Sevean, P., Dampier, S., Spadoni, M., Strickland, S., and Pilatzke, S. 2008. "Bridging the Distance: Educating Nurses for Telehealth Practice," The Journal of Continuing Education in Nursing (39:9), pp. 413-418.

Shahpori, R., Hebert, M., Kushniruk, A., and Zuege, D. 2011. "Telemedicine in the Intensive Care Unit Environment - a Survey of the Attitudes and Perspectives of Critical Care Clinicians," Journal of critical care (26:3), pp. 328. e329-328. e315.

Sherer, S. A. 2014. "Advocating for Action Design Research on It Value Creation in Healthcare," Journal of the Association for Information Systems (15:12), p. 860.

Singh, R., Mathiassen, L., Stachura, M. E., and Astapova, E. V. 2011. "Dynamic Capabilities in Home Health: It-Enabled Transformation of Post-Acute Care*," Journal of the Association for Information Systems (12:2), p. 163. 
Smith, W. K., and Lewis, M. W. 2011. "Toward a Theory of Paradox: A Dynamic Equilibrium Model of Organizing," Academy of Management Review (36:2), pp. 381-403.

Srivastava, S. C., and Shainesh, G. 2015. "Bridging the Service Divide through Digitally Enabled Service Innovations: Evidence from Indian Healthcare Service Providers," Mis Quarterly (39:1), pp. 245-267.

Stange, K. C. 2009. "The Problem of Fragmentation and the Need for Integrative Solutions," The Annals of Family Medicine (7:2), pp. 100-103.

Stewart, M. J., Georgiou, A., and Westbrook, J. I. 2013. "Successfully Integrating Aged Care Services: A Review of the Evidence and Tools Emerging from a Long-Term Care Program," International journal of integrated care (13:1).

Stille, C. J., Jerant, A., Bell, D., Meltzer, D., and Elmore, J. G. 2005. "Coordinating Care across Diseases, Settings, and Clinicians: A Key Role for the Generalist in Practice," Annals of internal medicine (142:8), pp. 700-708.

Tan, B., Pan, S. L., Lu, X., and Huang, L. 2015. "The Role of Is Capabilities in the Development of Multi-Sided Platforms: The Digital Ecosystem Strategy of Alibaba. Com," Journal of the Association for Information Systems (16:4), p. 248.

Tiwana, A., Konsynski, B., and Bush, A. A. 2010. "Research Commentary-Platform Evolution: Coevolution of Platform Architecture, Governance, and Environmental Dynamics," Information Systems Research (21:4), pp. 675-687.

Toh, H., Chia, J., Koh, E., Lam, K., Magpantay, G., De Leon, C., and Low, J. 2015. "Virtual Geriatric Care: User Perception of Telegeriatrics in Nursing Homes of Singapore," in Information and Communication Technologies for Ageing Well and E-Health. Springer, pp. 91-105.

Tsasis, P., Evans, J. M., and Owen, S. 2012. "Reframing the Challenges to Integrated Care: A Complex-Adaptive Systems Perspective," International Journal of Integrated Care (12:5).

Vargo, S. L., and Lusch, R. F. 2004. "Evolving to a New Dominant Logic for Marketing," Journal of marketing (68:1), pp. 1-17.

Vargo, S. L., and Lusch, R. F. 2008. "Service-Dominant Logic: Continuing the Evolution," Journal of the Academy of marketing Science (36:1), pp. 1-10.

Wareham, J., Fox, P. B., and Cano Giner, J. L. 2014. "Technology Ecosystem Governance," Organization Science (25:4), pp. 1195-1215.

Weill, P., and Woerner, S. L. 2015. "Thriving in an Increasingly Digital Ecosystem," MIT Sloan Management Review (56:4), p. 27.

Weinberger, M., Oddone, E. Z., and Henderson, W. G. 1996. "Does Increased Access to Primary Care Reduce Hospital Readmissions?," New England Journal of Medicine (334:22), pp. 1441-1447.

West, J., and Wood, D. 2013. "Evolving an Open Ecosystem: The Rise and Fall of the Symbian Platform," Advances in strategic management (30), pp. 27-67.

Whittaker, F., and Wade, V. 2014. "The Costs and Benefits of Technology-Enabled, HomeBased Cardiac Rehabilitation Measured in a Randomised Controlled Trial," Journal of telemedicine and telecare (20:7), pp. 419-422.

Yeow, A., and Goh, K. H. 2015. "Work Harder or Work Smarter? Information Technology and Resource Allocation in Healthcare Processes," Mis Quarterly (39:4), p. 12135_RA_YeowGoh.

Yin, R. 1994. "Case Study Research: Design and Methods . Beverly Hills." CA: Sage publishing. 
Yin, R. 2003. "K.(2003). Case Study Research: Design and Methods," Sage Publications, Inc (5), p. 11.

Zahra, S. A., and Nambisan, S. 2012. "Entrepreneurship and Strategic Thinking in Business Ecosystems," Business Horizons (55:3), pp. 219-229.

Zeng, M. 2015. "Three Paradoxes of Building Platforms," Communications of the ACM (58:2), pp. 27-29. 